\title{
Communication-Safe Web Programming in TypeScript with Routed Multiparty Session Types
}

\author{
Anson Miu \\ Imperial College London and Bloomberg \\ United Kingdom \\ Nobuko Yoshida \\ Imperial College London \\ United Kingdom
}

\author{
Francisco Ferreira \\ Imperial College London \\ United Kingdom \\ Fangyi Zhou \\ Imperial College London \\ United Kingdom
}

\begin{abstract}
Modern web programming involves coordinating interactions between browser clients and a server. Typically, the interactions in web-based distributed systems are informally described, making it hard to ensure correctness, especially communication safety, i.e. all endpoints progress without type errors or deadlocks, conforming to a specified protocol.

We present STScript, a toolchain that generates TypeScript APIs for communication-safe web development over WebSockets, and RouST, a new session type theory that supports multiparty communications with routing mechanisms.

STScript provides developers with TypeScript APIs generated from a communication protocol specification based on RouST. The generated APIs build upon TypeScript concurrency practices, complement the event-driven style of programming in full-stack web development, and are compatible with the Node.js runtime for server-side endpoints and the React.js framework for browser-side endpoints.

RouST can express multiparty interactions routed via an intermediate participant. It supports peer-to-peer communication between browser-side endpoints by routing communication via the server in a way that avoids excessive serialisation. RouST guarantees communication safety for endpoint web applications written using STScript APIs.

We evaluate the expressiveness of STScript for modern web programming using several production-ready case studies deployed as web applications.
\end{abstract}

CCS Concepts: • Software and its engineering $\rightarrow$ Source code generation; $\bullet$ Theory of computation $\rightarrow$ Distributed computing models.

Keywords: TypeScript, WebSocket, API generation, session types, deadlock freedom, web programming

CC '21, March 2-3, 2021, Virtual, USA

(c) 2021 Association for Computing Machinery.

This is the author's version of the work. It is posted here for your personal use. Not for redistribution. The definitive Version of Record was published in Proceedings of the 30th ACM SIGPLAN International Conference on Compiler Construction (CC '21), March 2-3, 2021, Virtual, USA, https://doi.org/10.1145/ 3446804.3446854.

\section{ACM Reference Format:}

Anson Miu, Francisco Ferreira, Nobuko Yoshida, and Fangyi Zhou. 2021. Communication-Safe Web Programming in TypeScript with Routed Multiparty Session Types. In Proceedings of the 30th ACM SIGPLAN International Conference on Compiler Construction (CC '21), March 2-3, 2021, Virtual, USA. ACM, New York, NY, USA, 27 pages. https://doi.org/10.1145/3446804.3446854

\section{Introduction}

Web technology advancements have changed the way people use computers. Many services that required standalone applications, such as email, chat, video conferences, or even games, are now provided in a browser. While the Hypertext Transfer Protocol (HTTP) is widely used for serving web pages, its Request-Response model limits the communication patterns - the server may not send data to a client without the client first making a request.

The WebSocket Protocol [12] addresses this limitation by providing a bi-directional channel between the client and the server, akin to a Unix socket. Managing the correct usage of WebSockets introduces an additional concern in the development process, due to a lack of WebSocket testing tools, requiring an (often ad-hoc) specification of the communication protocol between server and clients.

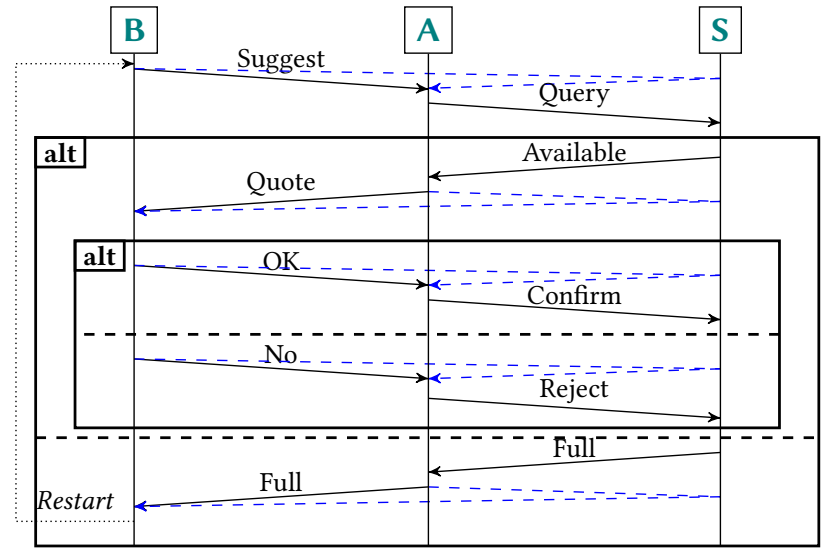

Figure 1. Travel Agency Protocol as a Sequence Diagram

Consider the scenario in Fig. 1, where an online travel agency operates a "travelling with a friend" scheme (ignoring 
the blue dashed arrows). It starts when a traveller (B) suggests a trip destination to their friend (A), who then queries the travel agency (S) if the trip is available. If so, the friends discuss among themselves whether to accept or reject the quoted price. If the trip was unavailable, the friends start again with a new destination.

An implementation of the travel agency protocol may contain programming errors, risking communication safety. For example, the following implementation of the client-side endpoint for traveller A sending a quote to traveller B.

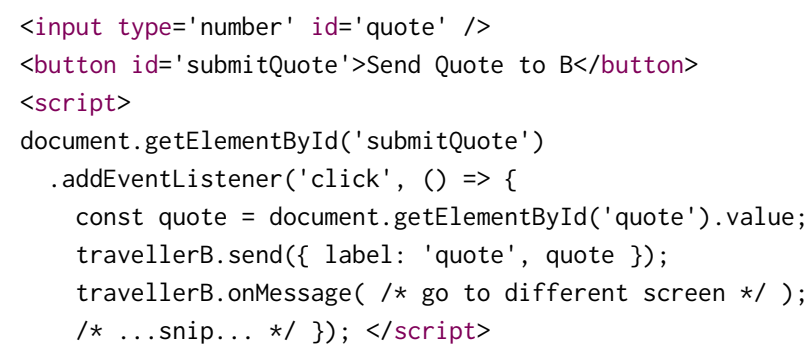

There are subtle errors that violate the communication protocol, but these bugs are unfortunately left for the developer to manually identify and test against:

Communication Mismatch Whilst the input field mandates a numerical value (Line 1) for the quote, the value from the input field is actually a string. If B expects a number and performs arithmetic operations on the received payload from A, the type mismatch may be left hidden due to implicit type coercion and cause unintended errors.

Channel Usage Violation As B may take time to respond, A can experience a delay between sending the quote and receiving a response. Notice that the button remains active after sending the quote - A could click on the button again, and send additional quotes (thus reusing the communication channel), but B may be unable to deal with extra messages. Handling Session Cancellation An additional concern is how to handle browser disconnections, as both travellers can freely close their browsers at any stage of the protocol. Suppose $\mathbf{S}$ temporarily reserves a seat on A's query. If A closes their browser, the developer would need to make sure that $A$ notifies $S$ prior to disconnecting, and $S$ needs to implement recovery logic (e.g. releasing the reserved seat) accordingly.

To prevent these errors and ensure deadlock-freedom, we propose to apply session types $[14,15]$ into practical interactive web programming. The scenario described in Fig. 1 can be precisely described with a global type using the typing discipline of multiparty session types (MPST) [15]. Well-typed implementations conform to the given global protocol, are guaranteed free from communication errors by construction.

Whereas session type programming is well-studied [1], its application on web programming, in particular, interactive web applications, remains relatively unexplored. Integrating session types with web programming has been piloted by recent work $[13,20,24]$, yet none are able to seamlessly implement the previous application scenario: Fowler [13] uses binary (2-party) session types; and King et al. [20] require each non-server role to only communicate to the server, hence preventing interactions between non-server roles (cf. talking to a friend in the scenario). The programming languages used in these works are, respectively, Links [8] and PureScript [28], both not usually considered mainstream in the context of modern web programming. The Jolie language [24] focuses more on the server side, with limited support for an interactive front end of web applications.

This paper presents a novel toolchain, Session TypeScript (STScript), for implementing multiparty protocols safely in web programming. STScript integrates with modern tools and practices, utilising the popular programming language TypeScript, front end framework React.js and back end runtime Node.js. Developers first specify a multiparty protocol and we generate correct-by-construction APIs for developers to implement the protocol. The generated APIs use WebSocket to establish communication between participants, utilising its flexibility over the traditional HTTP model. When developers use our generated APIs to correctly implement the protocol endpoints, STScript guarantees the freedom from communication errors, including deadlocks, communication mismatches, channel usage violation or cancellation errors.

Our toolchain is backed by a new session theory, a routed multiparty session types theory (RouST), to endow servers with the capacity to route messages between web clients. The new theory addresses a practical limitation that WebSocket connections still require clients to connect to a prescribed server, constraining the ability for inter-client communication. To overcome this, our API routes inter-client messages through the server, improving the expressiveness over previous work and enabling developers to correctly implement multiparty protocols, as we show with blue dashed arrows in Fig. 1. In our travel agency scenario, the agency plays the server role: it will establish WebSocket channels with each participant, and be tasked with routing all the messages between the friends. We formalise this routing mechanism as RouST and prove deadlock-freedom of RouST and show a behaviour-preserving encoding from the original MPST to RouST. The formalism and results in RouST directly guide a deadlock-free protocol implementation in Node.js via the router, preserving communication structures of the original protocol written by a developer.

Finally, we evaluate our toolchain (STScript) by case studies. We evaluate the expressiveness by implementing a number of web applications, such as interactive multiplayer games (Noughts and Crosses, Battleship) and web services (Travel Agency) that require routed communication.

Contributions and Structure of the Paper. $\S 2$ presents an overview of our toolchain STScript, which generates APIs for communication-safe web applications in TypeScript from multiparty protocol descriptions. $\S 3$ motivates how the generated code executes the multiparty protocol descriptions, 


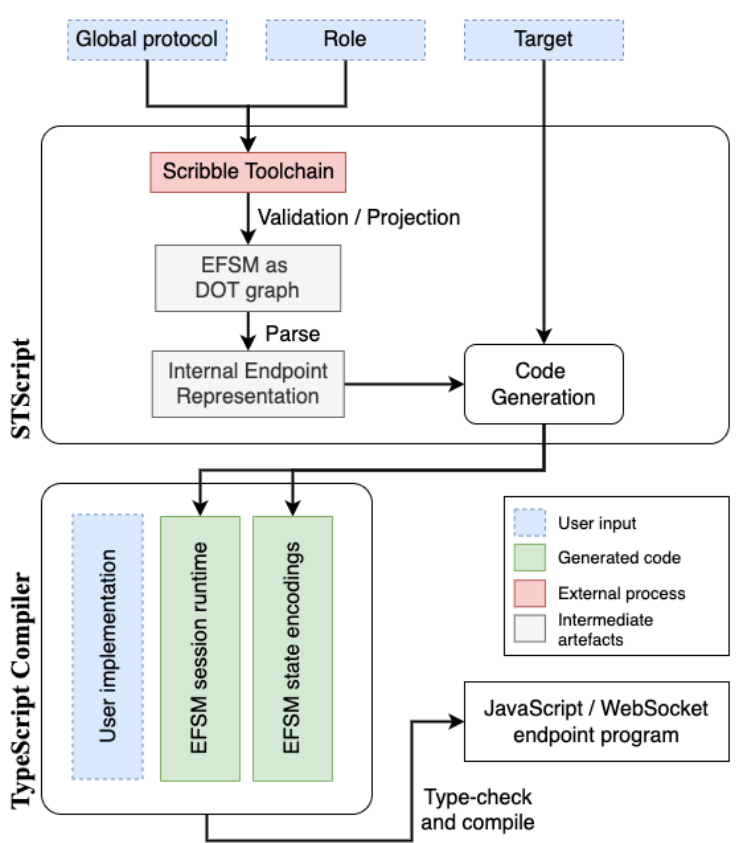

Figure 2. Overview of the toolchain STScript

and present how STScript prevents common errors in the context of web applications. § 4 presents RouST, multiparty session types (MPST) extended with routing, and define a trace-preserving encoding of the original MPST into RouST. $\S 5$ evaluates our toolchain STScript via a case study of Noughts and Crosses and performance experiments. § 6 gives related and future work.

Appendix includes omitted code, definitions, performance benchmarks and detailed proofs. The artifact accompanying this paper [23] is available via DOI or at https://github.com/ STScript-2020/cc21-artifact, containing the source code of STScript, with implemented case studies and performance benchmarks. See Appendix A for details about the artifact.

\section{Overview}

In this section, we give an overview of our code generation toolchain STScript (Fig. 2), demonstrate how to implement the travel agency scenario (Fig. 1) as a TypeScript web application, and explain how STScript prevents those errors.

Multiparty Session Type Design Workflow. Multiparty session types (MPST) [15] use a top-down design methodology (Fig. 3). Developers begin with specifying the global communication pattern of all participants in a global type or a global protocol. The protocol is described in the Scribble protocol description language $[16,31,34]$. We show the global protocol of the travel agency scenario (in § 1) in Fig. 4.

The Scribble language provides a user-friendly way to describe the global protocol in terms of a sequence of message exchanges between roles. A message is identified by its label (e.g. Suggest, Query, etc), and carries payloads (e.g. number, string, etc). The choice syntax (e.g. Line 4) describes possible branches of the protocol - in this case, the Server may

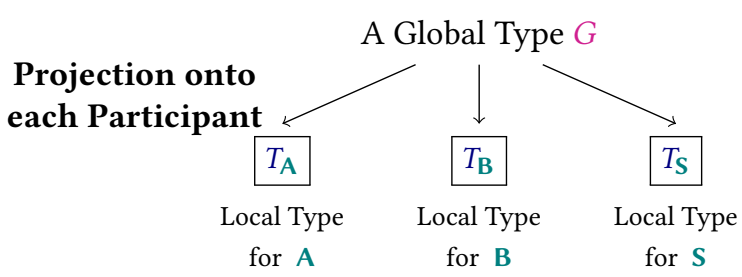

Figure 3. Top-down MPST Design Methodology

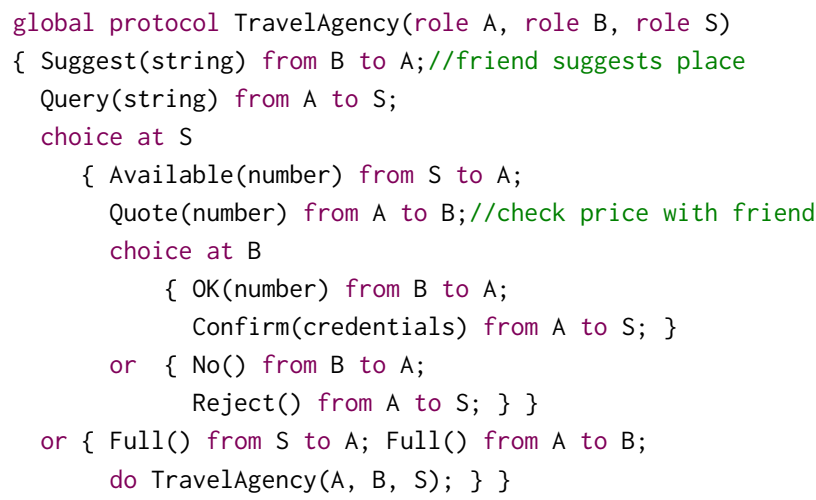

Figure 4. Travel Agency Protocol in Scribble

respond to the query either with Avai lable, so the customer continues booking, or with Full, so the customer retries by restarting the protocol via the do syntax (Line 13).

In this scenario, we designate the roles $\mathrm{A}$ and $\mathrm{B}$ as client roles, and role $\mathrm{S}$ as a server role. Participating endpoints can obtain their local views of the communication protocol, known as local types, via projection from the specified global type (Fig. 3). The local type of an endpoint can be then used in the code generation process, to generate APIs that are correct by construction [17, 20,35].

The code generation toolchain STScript (Fig. 2) follows the MPST design philosophy. In STScript, we take the global protocol as inputs, and generate endpoint code for a given role as outputs, depending on the nature of the role. We use the Scribble toolchain for initial processing, and use an endpoint finite state machine (EFSM) based code generation technique targeting the TypeScript Language.

Targeting Web Programming. The TypeScript [2] programming language is used for web programming, with a static type system and a compiler to JavaScript. TypeScript programs follow a similar syntax to JavaScript, but may contain type annotations that are checked statically by the TypeScript type-checker. After type-checking, the compiler converts TypeScript programs into JavaScript programs, so they can be run in browsers and other hosts (e.g. Node.js).

To implement a wide variety of communication patterns, we use the WebSocket protocol [12], enabling bi-directional communication between the client and the server after connection. This contrasts with the traditional request-response model of HTTP, where the client needs to send a request and the server may only send a response after receiving the 


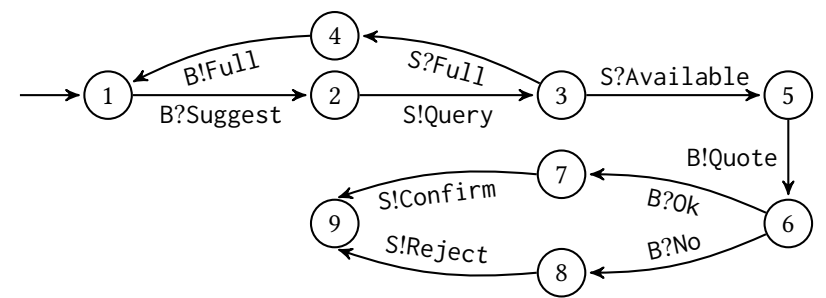

Figure 5. EFSM for TravelAgency role A

request. WebSockets require an endpoint to listen for connections and the other endpoint connecting. Moreover, clients, using the web application in a browser, may only start a connection to a WebSocket, and servers may only listen for new connections. The design of WebSocket limits the ability for two clients to communicate directly via a WebSocket (e.g. Line 2 in Fig. 4). STScript uses the server to route messages between client roles, enabling communication between all participants via a star network topology.

An important aspect of web programming is the interactivity of the user interface (UI). Viewed in a browser, the web application interacts with the user via UI events, e.g. mouse clicks on buttons. The handling of UI events may be implemented to send messages to the client (e.g. when the "Submit" button on the form is clicked), which may lead to practical problems. For instance, would clicking "Submit" button twice create two bookings for the customer? We use the popular React.js UI framework for generating client endpoints, and generate APIs that prevent such errors from happening.

Callback-Style API for Clients and Servers. Our code generation toolchain STScript produces TypeScript APIs in a callback style [35] to statically guarantee channel linearity. The input global protocol is analysed by the toolchain for well-formedness, and an endpoint finite state machine (EFSM) is produced for each endpoint. We show the EFSM for role A in Fig. 5. The states in the EFSM represent local types (subject to reductions) and transitions represent communication actions (Symbol ! stands for sending actions, ? for receiving).

In the callback API style, type signatures of callbacks are generated for transitions in the EFSM. Developers implement the callbacks to complete the program logic part of the application, whilst a generated runtime takes care of the communication aspects. For callbacks, sending actions correspond to callbacks prompting the payload type as a return type, so that the returned value can be sent by the runtime. Dually, receiving actions correspond to callbacks taking the payload type as an argument, so that the runtime invokes the callback with the received value.

Implementing the Server Role. In the travel agency protocol, as shown in Fig. 4, we designate role $\mathbf{S}$ as the server role. The server role does not only interact with the two clients, but also routes messages for the two clients. The routing will be handled automatically by the runtime, saving the need for developers to specify manually. As a result, the developer only handles the program logic regarding the

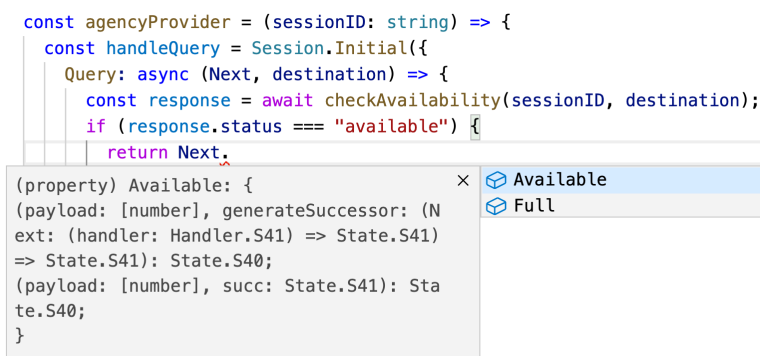

Figure 6. IDE Auto-Completion for Successor State

server, in this use case, namely providing quotes for holiday bookings and handling booking confirmations.

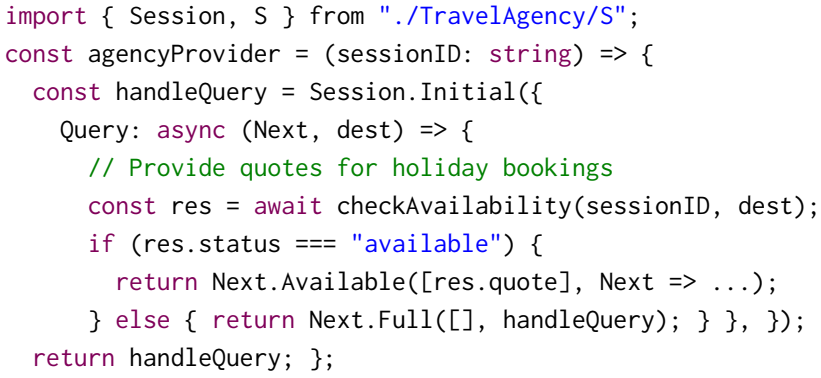

All callbacks carry an extra parameter, Next, which acts as a factory function for constructing the successor state. This empowers IDEs to provide auto-completion for developers. For example, the factory function provided by the callback for handling a Query message (Line 4) prompts the permitted labels in the successor send state, as illustrated in Fig. 6.

Implementing the Client Roles. To implement client roles, merely implementing the callbacks for the program logic is not sufficient - unlike servers, web applications have interactive user interfaces, additional to program logic. As mentioned previously, our code generation toolchain targets React.js for client roles. For background, the smallest building blocks in React.js are components, which can carry properties (immutable upon construction) and states (mutable). Components are rendered into HTML elements, and they are re-rendered when the component state mutates.

To bind the program logic with an interactive user interface, we provide component factories that allow the UI component to be interposed with the current state of the EFSM. Developers can provide the UI event handler to the component factory, and obtain a component for rendering. The generate code structure enforces that the state transition strictly follows the EFSM, so programmer errors (such as the double "submit" problem) are prevented by design.

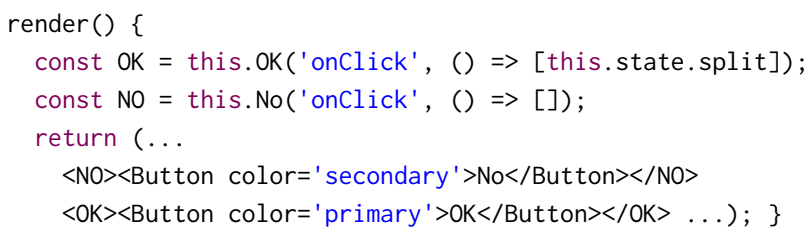


Using the send state component in the FSM for the endpoint B as an example, Line 2 reads, "generate a React component that sends the OK message with this.state.split as payload on a click event". It is used on Line 6 as a wrapper for a stylised <Button> component. The runtime invokes the handler and performs the state transition, which prevents the double "submit" problem by design.

Guaranteeing Communication Safety. Returning to the implementation in $\S 1$, we outline how STScript prevents common errors to enable type-safe web programming.

Communication Mismatch All generated callbacks are typed according to the permitted payload data type specified in the protocol, making it impossible for traveller $A$ to send the quote as a string by accident.

Channel Usage Violation The generated client-side runtime requires the developer to provide different UI components for each EFSM state - once traveller A submits a quote, the runtime will transition to, thus render the component of, a different EFSM state. This guarantees that, whilst waiting for a response from traveller $\mathbf{B}$, it is impossible for traveller A to submit another quote and violate channel linearity.

Handling Session Cancellation If either traveller closes their browser before the protocol runs to completion, the generated runtimes leverage the events available on their WebSocket connections to notify (via the server) other roles about the session cancellation. The travel agency can implement the error handler callback (generated by STScript) to perform clean-up logic in response to cancellations.

\section{Implementation}

In this section, we explain how the generated code executes the EFSM for Node.js and React.js targets. We also present how STScript APIs handle errors in a dynamic web-based environment (for complete code, see Appendix E).

Session Runtime. The session runtime executes the EFSM in a manner permitted by the multiparty protocol description. The runtime keeps track of the current state, performs the required communication action (i.e. send or receive a message), and transitions to the successor state. The runtime provides seams for the developer to inject the callback implementations, which define application-specific concerns for the EFSM, such as what message payload to send (and dually, how to process a received message). This design conceals the WebSocket APIs from the developer and entails that the developer cannot trigger a send or receive action, so STScript can statically guarantee protocol conformance.

Executing the EFSM in Node.js. Each state of the EFSM is characterised by a (generated) State class and a type describing the shape of the callback (supplied by the developer). To allow the server to correctly manage concurrent sessions, the developer can access a (generated) session ID when implementing the callbacks. STScript also generates IO interfaces for each kind of EFSM state - send, receive, or terminal. The generated State class implements the interface corresponding to the type of communication action it performs.

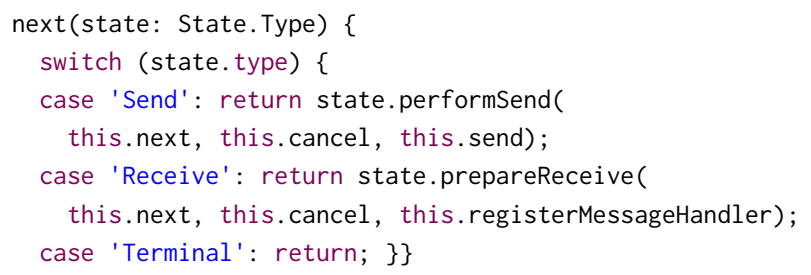

The session runtime for Node.js is a class that executes the EFSM using a state transition function parameterised by the State class of the current EFSM state. As the IO interfaces constitute a discriminated union, the runtime can parse the type of the current EFSM state and propagate the appropriate IO functions (for sending or receiving) to the State class. In turn, the State class invokes the callback supplied by the developer to inject program logic into the EFSM, perform the communication action (using this.send or this.registerMessageHandler), and invoke the state transition function (this. next) with the successor state.

Notably, the routed messages are completely absent because the generated code transparently routes messages without exposing any details. As messages specify their intended recipient, the runtime identifies messages not intended for the server by inspecting the metadata, and forwards them to the WebSocket connected to the intended recipient.

Executing the EFSM in React.js. Each state in the EFSM is encoded as an abstract React component. The developer implements the EFSM by extending the abstract classes to provide their own implementation - namely, to build their user interface. Components for send states can access component factories to generate React components that perform a send action when a UI event (e.g. onClick, onMouseOver) is triggered. Components for receive states must implement abstract methods to handle all possible incoming messages.

The session runtime for React.js is a React component, instantiated using the developer's implementation of each EFSM state. Channel communications are managed by the runtime, so the developer's implementations cannot access the WebSocket APIs, which prevents channel reuse by construction. The runtime renders the component of the current EFSM state and binds the permitted communication action through supplying component properties.

Error Handling. An error handling mechanism is critical for web applications. Clients can disconnect from the session due to network connectivity issues or simply by closing the browser. Similarly, servers may also face connectivity issues.

Upon instantiating the session runtime, STScript requires developers to supply a cancellation handler to handle local exceptions (e.g. errors thrown by application logic) and global session cancellations (e.g. disconnection events by another 
endpoint). The session runtime detects cancellation by listening to the close event on the WebSocket connection, and invokes the cancellation handler with appropriate arguments on a premature close event. We parameterise the cancellation handlers with additional information (e.g. which role disconnected from the session, the reason for the disconnection) to let developers be more specific in their error handling logic.

Cancellation Handlers for Servers. Server endpoints define cancellation handlers through a function, parameterised by the session ID, the role which initiated the cancellation, and (optionally) the reason for the cancellation - if the server-side logic throws an exception, the handler can access the thrown error through the reason parameter.

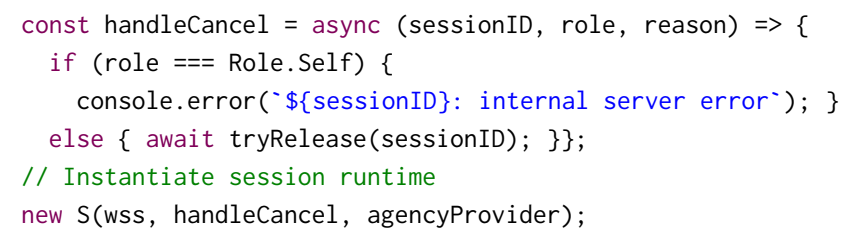

Using the Travel Agency scenario introduced in $\S 1$, if the customer prematurely closes their browser before responding to a Quote, the server can detect this (Line 4) and release the reservation to preserve data integrity.

Cancellation Handlers for Clients. Browser-side endpoints also define cancellation handlers through a function parameterised in the same way as those in Node.js, but must return a React component to be rendered by the session runtime. In the context of the Travel Agency scenario, the customer can render a different UI depending on whether the server disconnected or their friend closed their web browser prematurely. Browser endpoints can also respond to cancellations emitted by other client-side roles: when a browser endpoint disconnects, the server detects this and propagates the cancellation to the other client-side roles.

\section{RouST: Routed Session Types}

This section defines the syntax and semantics of RouST and proves some important properties. We show the sound and complete trace correspondence between a global type and a collection of endpoint types projected from the global type (Theorem 4.6). Using this result, we prove deadlock freedom (Theorem 4.7). We then show that, in spite of the added routed communications, RouST does not over-serialise communications by proving communication preservations between the original MPST and RouST (Theorem 4.11). These three theorems ensure that STScript endpoint programs are communication-safe, always make progress, and correctly conforms to the user-specified protocol.

\subsection{Syntax of Routed Multiparty Session Types}

We define the syntax of global types $G$ and local types (or endpoint types) $T$ in Definition 4.1. Global types are also known as protocols and describe the communication behaviour between all participating roles (participants), while local types describe the behaviour of a single participating role. We shade additions to the original (or canonical) multiparty session type (MPST) $[9,11,15,30]$ in this colour .

Definition 4.1 (Global and Local Types). The syntax of global and local types are defined below:

$$
\begin{aligned}
& G::=\text { end }|\mathbf{t}| \mu \mathbf{t} . G \quad T::=\text { end }|\mathbf{t}| \mu \mathbf{t} . T \mid \mathbf{p} \hookrightarrow \mathbf{q}:\left\{l_{i}: T_{i}\right\}_{i \in I} \\
& \left|\mathbf{p} \rightarrow \mathbf{q}:\left\{l_{i}: G_{i}\right\}_{i \in I} \quad\right| \mathbf{p} \oplus\left\{l_{i}: T_{i}\right\}_{i \in I} \quad \mid \mathbf{p} \oplus\langle\mathbf{q}\rangle\left\{l_{i}: T_{i}\right\}_{i \in I} \\
& \left|\mathbf{p}-\mathbf{s} \rightarrow \mathbf{q}:\left\{l_{i}: G_{i}\right\}_{i \in I} \quad\right| \mathbf{p} \&\left\{l_{i}: T_{i}\right\}_{i \in I} \quad \mid \mathbf{p} \&\langle\mathbf{q}\rangle\left\{l_{i}: T_{i}\right\}_{i \in I}
\end{aligned}
$$

Global Types. $\mathbf{p} \rightarrow \mathbf{q}:\left\{l_{i}: G_{i}\right\}_{i \in I}$ describes a direct communication of a message $l_{i}$ from a role $\mathrm{p}$ to $\mathrm{q}$. We require that $\mathbf{p} \neq \mathbf{q}$, that labels $l_{i}$ are pairwise distinct, and that the index set $I$ is not empty. The message in the communication can carry a label among a set of permitted labels $l_{i}$ and some payload. After a message with label $l_{i}$ is received by $\mathbf{q}$, the communication continues with $G_{i}$, according to the chosen label. For simplicity, we do not include payload types (integers, strings, booleans, etc) in the syntax. We write $\mathbf{p} \rightarrow \mathbf{q}: l: G$ for single branches. For recursion, we adopt an equi-recursive view [27, §21], and use $\mu \mathrm{t} . G$ and tor a recursive protocol and a type variable. We require that recursive types are contractive (guarded), i.e. the recursive type $\mu \mathrm{t}$. $G$ progresses after the substitution $G[\mu \mathrm{t} . G / \mathrm{t}]$, prohibiting types such as $\mu$ t.t. We use end to mark the termination of the protocol, and often omit the final end.

To support routed communication, we allow messages to be sent through a router role. A routed communication $\mathbf{p}-\mathbf{s} \rightarrow \mathbf{q}:\left\{l_{i}: G_{i}\right\}_{i \in I}$ describes a router role $\mathbf{s}$ coordinating the communication of a message from $p$ to $q$ : $q$ offers $p$ a choice in the index set $I$, but $\mathrm{p}$ sends the selected choice $l_{i}$ to the router $s$ instead. The router forwards the selection from p to q. After q receives p's selection, the communication continues with $G_{i}$. s ranges over the set of roles $\mathbf{p}, \mathbf{q}, \cdots$, but we use s by convention as the router is usually some server. The syntax for routed communication shares the same properties as direct communication, but we additionally require that $\mathbf{p} \neq \mathbf{q} \neq \mathbf{s}$. We use $\mathbf{p t}(G)$ to denote the set of participants in the global type $G$.

Example 4.2 (Travel Agency). The travel agency protocol, as shown in Fig. 4, is described by the global type $G_{\text {travel }}$ in the original MPST, and $G_{\text {travel }}^{R}$ in RouST.

$$
\begin{aligned}
& G_{\text {travel }}=\mu \mathrm{t} . \mathbf{B} \rightarrow \mathbf{A}: \text { Suggest } . \mathbf{A} \rightarrow \mathbf{S}: \text { Query } . \\
& \mathbf{S} \rightarrow \mathbf{A}:\left\{\begin{array}{c}
\text { Available }: \\
\mathbf{A} \rightarrow \mathbf{B}: \text { Quote } \mathbf{B} \rightarrow \mathbf{A}: \\
\left\{\begin{array}{l}
\text { OK }: \mathbf{A} \rightarrow \mathbf{S}: \text { Confirm } \\
\text { No }: \mathbf{A} \rightarrow \mathbf{S}: \text { Reject }
\end{array}\right\} \\
\text { Full }: \mathbf{A} \rightarrow \mathbf{B}: \text { Full } . \mathbf{t}
\end{array}\right\} \\
& G_{\text {travel }}^{R}=\mu \mathrm{t} . \mathbf{B}-\mathbf{S} \rightarrow \mathbf{A}: \text { Suggest } . \mathbf{A} \rightarrow \mathbf{S}: \text { Query . } \\
& \mathbf{S} \rightarrow \mathbf{A}:\left\{\begin{array}{c}
\text { Available }: \\
\mathbf{A}-\mathbf{S} \rightarrow \mathbf{B}: \text { Quote } \mathbf{B}-\mathbf{S} \rightarrow \mathbf{A}: \\
\left\{\begin{array}{l}
\text { OK }: \mathbf{A} \rightarrow \mathbf{S}: \text { Confirm } \\
\text { No: }: \mathbf{A} \rightarrow \mathbf{S}: \text { Reject }
\end{array}\right. \\
\text { Full }: \mathbf{A}-\mathbf{S} \rightarrow \mathbf{B}: \text { Full } . \mathbf{t}
\end{array}\right\}
\end{aligned}
$$


Local Types. We first describe the local types in the original MPST theory. q \& $\left\{l_{i}: T_{i}\right\}_{i \in I}$ stands for branching and $\mathbf{q} \oplus\left\{l_{i}: T_{i}\right\}_{i \in I}$ stands for selection. From the perspective of p, branching (resp. selection) offers (resp. selects) a choice among an index set $I$ to (resp. from) $\mathrm{q}$, and communication continues with the corresponding $T_{i}$. Local types $\mu \mathbf{t} . T$, $\mathbf{t}$ and end have the same meaning as their global type counterparts.

We add new syntax to express routed communication from the perspective of each role involved. The local type $\mathrm{p} \&\langle\mathrm{~s}\rangle\left\{l_{i}: T_{i}\right\}_{i \in I}$ is a routed branching: the current role is offering a choice from an index set $I$ to $\mathrm{p}$ (the intended sender), but expects to receive p's choice via the router role $\mathrm{s}$; if the message received is labelled $l_{i}$, q will continue with local type $T_{i}$. The local type $\mathbf{q} \oplus\langle\mathbf{s}\rangle\left\{l_{i}: T_{i}\right\}_{i \in I}$ is a routed selection: the current role makes a selection from an index set $I$ to $\mathrm{q}$ (the intended recipient), but sends the selection to the router role $s$; if the message sent is labelled $l_{i}, \mathbf{p}$ will continue with local type $T_{i}$. The local type $\mathbf{p} \hookrightarrow \mathbf{q}:\left\{l_{i}: T_{i}\right\}_{i \in I}$ is a routing communication. The router role orchestrates the communication from $\mathbf{p}$ to $\mathbf{q}$, and continues with local type $T_{i}$ depending on the label of the forwarded message. We keep track of the router role to distinguish between routing communications from normal selection and branching interactions.

Endpoint Projection. The local type $T$ of a participant $p$ in a global type $G$ is obtained by the endpoint projection of $G$ onto $\mathbf{p}$, denoted by $G$ as $G \uparrow \mathbf{p}$.

Definition 4.3 (Projection). The projection of $G$ onto $r$, written $G \uparrow r$ is defined as:

$$
\begin{aligned}
& \left(\mathbf{p}-\mathbf{s} \rightarrow \mathbf{q}:\left\{l_{i}: G_{i}\right\}_{i \in I}\right) \uparrow \mathbf{r}
\end{aligned}
$$

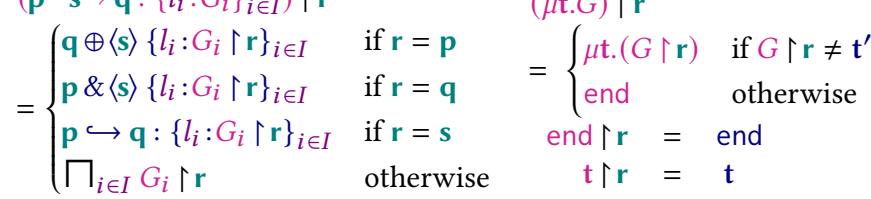

The projection $\left(\mathbf{p} \rightarrow \mathbf{q}:\left\{l_{i}: G_{i}\right)\right\}_{i \in I} \uparrow \mathbf{r}$ is defined similar to $\left(\mathbf{p}-\mathbf{s} \rightarrow \mathbf{q}:\left\{l_{i}: G_{i}\right\}_{i \in I}\right) \uparrow \mathbf{r}$ dropping $\mathbf{s}$ (in the resulting local type) and the third case.

A merge operator $(\sqcap)$ is used when projecting a communication onto a non-participant. It checks that the projections of all continuations must be "compatible" (see Definition B.2).

Example 4.4 (Merging Local Types). Two branching types from the same role with disjoint labels can merged into a type carrying both labels, e.g. A\& Hello.end $\square$ A \& Bye.end $=$ A \& $\{$ Hello : end; Bye : end $\}$. The same is not true for selections, $\mathrm{A} \oplus$ Hello.end $\sqcap \mathrm{A} \oplus$ Bye.end is undefined.

$$
\begin{gathered}
G_{1}=\mathbf{A} \rightarrow \mathbf{B}:\left\{\begin{array}{l}
\text { Greet }: \mathbf{A} \rightarrow \mathbf{C}: \text { Hello .end } \\
\text { Farewell }: \mathbf{A} \rightarrow \mathbf{C}: \text { Bye .end }
\end{array}\right\} \\
G_{2}=\mathbf{A} \rightarrow \mathbf{B}:\left\{\begin{array}{l}
\text { Greet }: \mathbf{C} \rightarrow \mathbf{A}: \text { Hello .end } \\
\text { Farewell }: \mathbf{C} \rightarrow \mathbf{A}: \text { Bye .end }
\end{array}\right\}
\end{gathered}
$$

The global type $G_{1}$ can be projected to role C, but not $G_{2}$.
Well-formedness. In the original theory, a global type $G$ is well-formed (or realisable), denoted wellFormed $(G)$, if the projection is defined for all its participants.

$$
\text { wellFormed }(G) \stackrel{\text { def }}{=} \forall p \in \text { pt }(G) \text {. } G \uparrow p \text { exists }
$$

We assume that the global type $G$ is contractive (guarded).

In RouST, we say that a global type is well-formed with respect to the role s acting as the router. We define the characteristics that $\mathbf{s}$ must display in $G$ to prove that it is a router, and formalise this as an inductive relation, $G \circledast \mathbf{s}$ (Definition 4.5), which reads s is a centroid in $G$. The intuition is that $s$ is at the centre of all communication interactions.

Definition 4.5 (Centroid). The relation $G \circledast \mathrm{s}$ (s is the centroid of $G$ ) is defined by the two axioms end $\circledast \mathbf{s}$ and $\mathbf{t} \circledast \mathbf{s}$ and by the following rules:

$$
\frac{G \circledast \mathbf{s}}{\mu \mathbf{t} . G \circledast \mathbf{s}} \frac{\mathbf{s} \in\{\mathbf{p}, \mathbf{q}\} \quad \forall i \in I . G_{i} \circledast \mathbf{s}}{\mathbf{p} \rightarrow \mathbf{q}:\left\{l_{i}: G_{i}\right\}_{i \in I} \circledast \mathbf{s}} \frac{\mathbf{r}=\mathbf{s} \quad \forall i \in I . G_{i} \circledast \mathbf{s}}{\mathbf{p}-\mathbf{r} \rightarrow \mathbf{q}:\left\{l_{i}: G_{i}\right\}_{i \in I} \circledast \mathbf{s}}
$$

For direct communication, $s$ must be a participant and a centroid of all continuations. For routed communication, $s$ must be the router and be a centroid of all continuations. Now we define of well-formedness of a global type $G$ in RouST with respect to the router $\mathbf{s}$ (denoted wellFormed ${ }^{R}(G, \mathbf{s})$ ): wellFormed $^{R}(G, \mathbf{s}) \stackrel{\text { def }}{=}(\forall \mathbf{p} \in$ pt $(G) . G\lceil\mathbf{p}$ exists $) \wedge G \circledast \mathbf{s}$

\subsection{Semantics of RouST}

This subsection defines the labelled transition system (LTS) over global types for RouST, building upon [11].

First, we define the labels (actions) in the LTS which distinguish the direct sending (and reception) of a message from the sending (and reception) of a message via an intermediate routing endpoint. Labels range over $l, l^{\prime}, \cdots$ are defined by:

$$
l::=\mathbf{p q} ! j|\mathbf{p q} ? j| \mathrm{via}\langle\mathbf{s}\rangle(\mathbf{p q} ! j) \mid \mathrm{vi} a\langle\mathbf{s}\rangle(\mathbf{p q} ? j)
$$

The label $v i a\langle s\rangle(p q ! j)$ represents the sending (performed by $\mathbf{p}$ ) of a message labelled $j$ to $\mathbf{q}$ through the intermediate router s. The label $v i a\langle s\rangle(p q ? j)$ represents the reception (initiated by q) of a message labelled $j$ send from $p$ through the intermediate router $\mathrm{s}$. The subject of a label $l$, denoted by $\operatorname{subj}(l)$, is defined as: $\operatorname{subj}(\operatorname{via}\langle\mathbf{s}\rangle(\mathbf{p q} ! j))=\operatorname{subj}(\mathbf{p q} ! j)=\mathbf{p}$; and $\operatorname{subj}(\operatorname{via}\langle\mathbf{s}\rangle(\mathbf{p q} ? j))=\operatorname{subj}(\mathbf{p q} ? j)=\mathbf{q}$.

LTS Semantics over Global Types. The LTS semantics model asynchronous communication to reflect our implementation. We introduce intermediate states (i.e. messages in transit) within the grammar of global types: the construct $\mathbf{p} \rightsquigarrow \mathbf{q} . j:\left\{l_{i}: G_{i}\right\}_{i \in I}$ represents that the message $l_{j}$ has been sent by $\mathbf{p}$ but not yet received by $\mathbf{q}$; and the construct $\mathbf{p} \underset{\mathbf{s}}{\rightsquigarrow \text { q. }} j:\left\{l_{i}: G_{i}\right\}_{i \in I}$ represents that $l_{j}$ has been sent from $\mathrm{p}$ to the router $\mathrm{s}$ but not yet routed to $\mathrm{q}$. We define the LTS semantics over global types, denoted by $G \stackrel{l}{\longrightarrow} G^{\prime}$, in Fig. 7. [GR1] and [GR2] model the emission and reception of a message; [GR3] models recursions; [GR4] and [GR5] model causally unrelated transmissions - we only enforce the syntactic order of messages for the participants involved 


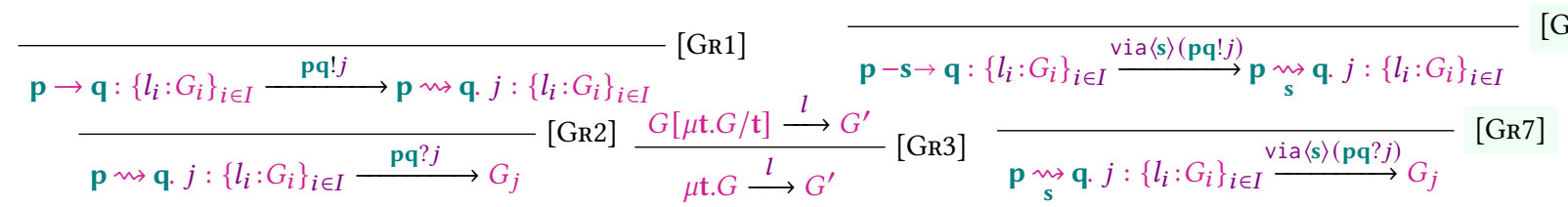

$$
\begin{aligned}
& \frac{\forall i \in I . G_{i} \stackrel{l}{\longrightarrow} G_{i}^{\prime} \quad \operatorname{subj}(l) \notin\{\mathbf{p}, \mathbf{q}\}}{\mathbf{p} \rightarrow \mathbf{q}:\left\{l_{i}: G_{i}\right\}_{i \in I} \stackrel{l}{\longrightarrow} \mathbf{p} \rightarrow \mathbf{q}:\left\{l_{i}: G_{i}^{\prime}\right\}_{i \in I}}[\mathrm{GR} 4] \\
& \frac{G_{j} \stackrel{l}{\longrightarrow} G_{j}^{\prime} \quad \operatorname{subj}(l) \neq \mathbf{q} \quad \forall i \in I \backslash\{j\} . G_{i}^{\prime}=G_{i}}{\mathbf{p} \rightsquigarrow \mathbf{q} \cdot j:\left\{l_{i}: G_{i}\right\}_{i \in I} \stackrel{l}{\longrightarrow} \mathbf{p} \rightsquigarrow \mathbf{q} \cdot j:\left\{l_{i}: G_{i}^{\prime}\right\}_{i \in I}}[\text { GR5] }
\end{aligned}
$$

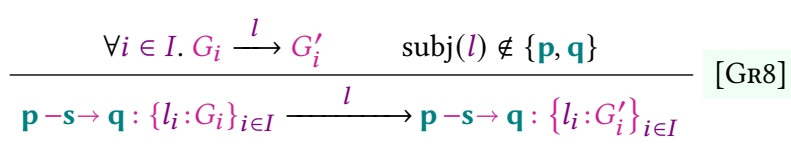

$$
\begin{aligned}
& \frac{G_{j} \stackrel{l}{\longrightarrow} G_{j}^{\prime} \quad \operatorname{subj}(l) \neq \mathbf{q} \quad \forall i \in I \backslash\{j\} . G_{i}^{\prime}=G_{i}}{\text { p } \underset{\mathbf{s}}{\mathbf{q}} \cdot j:\left\{l_{i}: G_{i}\right\}_{i \in I} \stackrel{l}{\longrightarrow} \underset{\mathbf{p}}{\mathbf{s}} \underset{\mathbf{q}}{\mathrm{q}} \cdot j:\left\{l_{i}: G_{i}^{\prime}\right\}_{i \in I}} \quad
\end{aligned}
$$

Figure 7. LTS over Global Types in RouST

in the action $l$. [GR6] and [GR7] are analogous to [GR1] and [GR2] for describing routed communication, but uses the "routed in-transit" construct instead. [GR8] and [GR9] are analogous to [GR4] and [GR5]. An important observation from [GR8] and [GR9] is that, for the router, the syntactic order of routed communication can be freely interleaved between the syntactic order of direct communication. This is crucial to ensure that the router does not over-serialise communication. See Example 4.13 for an LTS example.

Relating Semantics of Global and Local Types. We prove the soundness and completeness of our LTS semantics with respect to projection. We take three steps following [11]:

1. We extend the LTS semantics with configuration $(\vec{T}, \vec{w})$, a collection of local types $\vec{T}$ with FIFO queues between each pair of participants $\vec{w}$.

2. We extend the definition of projection, to obtain a configuration of a global type (a projected configuration), which expresses intermediate communication over FIFO queues.

3 . We prove the trace equivalence between the global type and its projected configuration (i.e. the initial configuration of $G,(\vec{T}, \vec{\epsilon})$, where $\vec{T}=\left\{G\lceil\mathbf{p}\}_{\mathbf{p} \in \mathcal{P}}\right.$ are a set of local types projected from $G$ and $\epsilon$ is an empty queue).

The proof is non-trivial: due to space limitations, we omit the semantics of local types, configurations and global configurations, and only state the main result (see Appendices B and C).

Theorem 4.6 (Sound and Complete Trace Equivalence). Let $G$ be a well-formed canonical global type. Then $G$ is trace equivalent to its initial configuration.

Theorem 4.7 proves traces specified by a well-formed global protocol are deadlock-free, i.e. the global type either completes all communications, or otherwise makes progress. Note that this theorem implies the deadlock-freedom of configurations by Theorem 4.6.

Theorem 4.7 (Deadlock Freedom). Let $G$ be a global type. Suppose $G$ is well-formed with respect to some router s, i.e.
wellFormed ${ }^{R}(G, \mathbf{s})$. Then we have:

$\forall G^{\prime} .\left(G \rightarrow^{*} G^{\prime} \Longrightarrow\left(G^{\prime}=\right.\right.$ end $\left.) \vee \exists G^{\prime \prime}, l .\left(G^{\prime} \stackrel{l}{\longrightarrow} G^{\prime \prime}\right)\right)$

\subsection{From Canonical MPST to RouST}

We present an encoding from the canonical MPST theory (no routers) to RouST. This encoding is parameterised by the router role (conventionally denoted as s); the intuition is that we encode all communication interactions to involve s. If the encoding preserves the semantics of the canonical global type, then this encoding can guide a correct protocol implementation in Node.js via s, preserving communication structures of the original protocol without deadlock.

Router-Parameterised Encoding. We define the routerparameterised encoding on global types, local types and LTS labels in the MPST theory. We start with global types, as presented in Definition 4.8. The main rule is the direct communication: if the communication did not go through s, then the encoded communication involves $s$ as the router.

Definition 4.8 (Encoding on Global Types). The encoding of global type $G$ with respect to the router role s, denoted by $\llbracket G, s \rrbracket$, is defined as:

$$
\begin{array}{r}
\llbracket \text { end, } \mathbf{s}=\text { end } \quad \llbracket \mathrm{t}, \mathrm{s} \rrbracket=\mathrm{t} \quad \llbracket \mu \mathrm{t} . G, \mathrm{~s} \rrbracket=\mu \mathrm{t} . \llbracket G, \mathrm{~s} \rrbracket \\
\llbracket \mathbf{p} \rightarrow \mathbf{q}:\left\{l_{i}: G_{i}\right\}_{i \in I}, \mathrm{~s} \rrbracket= \begin{cases}\mathbf{p} \rightarrow \mathbf{q}:\left\{l_{i}: \llbracket G_{i}, \mathrm{~s} \rrbracket\right\}_{i \in I} & \text { if } \mathbf{s} \in\{\mathbf{p}, \mathbf{q}\} \\
\mathbf{p}-\mathbf{s} \rightarrow \mathbf{q}:\left\{l_{i}: \llbracket G_{i}, \mathbf{s} \rrbracket\right\}_{i \in I} & \text { otherwise }\end{cases}
\end{array}
$$

Local types express communication from the perspective of a particular role, hence the encoding takes two roles.

Definition 4.9 (Encoding on Local Types). The encoding of local type $T$ (from the perspective of role q) with respect to the router role $\mathbf{s}$, denoted by $\llbracket T, \mathbf{q}, \mathbf{s} \rrbracket$, is defined as:

$$
\begin{gathered}
\llbracket \text { end, } \mathbf{q}, \mathbf{s} \rrbracket=\text { end } \llbracket \mathbf{t}, \mathbf{q}, \mathbf{s} \rrbracket=\mathbf{t} \llbracket \mu \mathbf{t} . T, \mathbf{q}, \mathbf{s} \rrbracket=\mu \mathbf{t} . \llbracket T, \mathbf{q}, \mathbf{s} \rrbracket \\
\llbracket \mathbf{p} \oplus\left\{l_{i}: T_{i}\right\}_{i \in I}, \mathbf{q}, \mathbf{s} \rrbracket= \begin{cases}\mathbf{p} \oplus\left\{l_{i}: \llbracket T_{i}, \mathbf{q}, \mathbf{s} \rrbracket\right\}_{i \in I} & \text { if } \mathbf{s} \in\{\mathbf{p}, \mathbf{q}\} \\
\mathbf{p} \oplus\langle\mathbf{s}\rangle\left\{l_{i}: \llbracket T_{i}, \mathbf{q}, \mathbf{s} \rrbracket\right\}_{i \in I} & \text { otherwise }\end{cases} \\
\llbracket \mathbf{p} \&\left\{l_{i}: T_{i}\right\}_{i \in I}, \mathbf{q}, \mathbf{s} \rrbracket= \begin{cases}\mathbf{p} \&\left\{l_{i}: \llbracket T_{i}, \mathbf{q}, \mathbf{s} \rrbracket\right\}_{i \in I} & \text { if } \mathbf{s} \in\{\mathbf{p}, \mathbf{q}\} \\
\mathbf{p} \&\langle\mathbf{s}\rangle\left\{l_{i}: \llbracket T_{i}, \mathbf{q}, \mathbf{s} \rrbracket\right\}_{i \in I} & \text { otherwise }\end{cases}
\end{gathered}
$$


Lemma 4.10 (Correspondence between Encodings). The projection of an encoded global type $\llbracket G, \mathrm{~s} \rrbracket\lceil\mathrm{r}$ is equal to the encoded local type after projection $\llbracket G \uparrow \mathrm{r}, \mathrm{r}, \mathrm{s} \rrbracket$, with respect to router $\mathrm{s}$, i.e. $\forall \mathrm{r}, \mathrm{s}, G .(\mathrm{r} \neq \mathrm{s} \Longrightarrow \llbracket G, \mathrm{~s} \rrbracket \uparrow \mathrm{r}=\llbracket G \uparrow \mathrm{r}, \mathrm{r}, \mathrm{s} \rrbracket)$.

The constraint $r \neq s$ is necessary because we would otherwise lose information on the right-hand side of the equality: the projection of $s$ in the original communication does not contain the routed interactions, so applying the local type encoding cannot recover this information.

Theorem 4.11 (Encoding Preserves Well-Formedness). Let $G$ be a global type, and $\mathrm{s}$ be a role. Then we have:

$$
\text { wellFormed }(G) \Longleftrightarrow \operatorname{wellFormed~}^{R}(\llbracket G, \mathbf{s} \rrbracket, \mathbf{s})
$$

Preserving Communication. We present a crucial result that directly addresses the pitfalls of naive definitions of routed communication - our encoding does not overserialise the original communication. We prove that our encoding preserves the LTS semantics over global types or more precisely, we can use the encodings over global types and LTS actions to encode all possible transitions in the LTS for global types in the canonical MPST theory. We define the encoding of label $l$ in the original MPST as: $\llbracket \mathbf{p q} ! j, s \rrbracket=v i a\langle s\rangle(\mathbf{p q} ! j)$ and $\llbracket \mathbf{p q} ? j, s \rrbracket=v i a\langle s\rangle(\mathbf{p q} ? j)$ if $\mathrm{s} \notin\{\mathrm{p}, \mathrm{q}\}$ and otherwise $\llbracket l, \mathrm{~s} \rrbracket=l$.

Theorem 4.12 (Encoding Preserves Semantics). Let $G, G^{\prime}$ be well-formed global types such that $G \stackrel{l}{\longrightarrow} G^{\prime}$ for some label l. Then we have:

$$
\forall l, \text { s. }\left(G \stackrel{l}{\longrightarrow} G^{\prime} \Longleftrightarrow \llbracket G, s \rrbracket \stackrel{\llbracket l, s \rrbracket}{\longrightarrow} \llbracket G^{\prime}, \mathrm{s} \rrbracket\right)
$$

We conclude with an example which demonstrates global semantics in RouST and a use of the encoding.

Example 4.13 (Encoding Preserves Semantics). Consider the global type

$$
G=\mathbf{p} \rightarrow \mathbf{q}: M 1 . \mathbf{s} \rightarrow \mathbf{q}: M 2 \text {. end. }
$$

We apply our encoding with respect to the router role s:

$$
\llbracket G, \mathbf{s} \rrbracket=\mathbf{p}-\mathbf{s} \rightarrow \mathbf{q}: M 1 . \mathbf{s} \rightarrow \mathbf{q}: M 2 \text {. end. }
$$

We note that $l=\mathbf{s q} ! M 2$ can reduce $G$ through [GR1] (via one application of [GR4]). After encoding, we have that $\llbracket l, \mathrm{~s} \rrbracket=l$. The encoded global type $\llbracket G, s \rrbracket$ can be reduced by $l$ through [GR1] (via one application of [GR8]), as demonstrated by Theorem 4.12. The label $l=\mathbf{s q !} M 2$ is a prefix of a valid execution trace for $G$, given below.

$$
G \stackrel{\mathrm{sq} ! M 2}{\longrightarrow} \stackrel{\mathrm{pq} ! M 1}{\longrightarrow} \stackrel{\mathrm{pq} ? M 1}{\longrightarrow} \stackrel{\text { sq? } ? M 2}{\longrightarrow} \text { end }
$$

Interested readers can verify that the encoded trace (given below) is a valid execution trace for $\llbracket G, s \rrbracket$.

$\llbracket G, \mathbf{s} \rrbracket \stackrel{\text { sq!M2 }}{\longrightarrow} \stackrel{v i a\langle s\rangle(\mathbf{p q} ! M 1)}{\longrightarrow} \stackrel{\text { via }\langle\mathbf{s}\rangle(\mathbf{p q} ? M 1)}{\longrightarrow} \stackrel{\text { sq?M2 }}{\longrightarrow}$ end

\section{Evaluation}

In this section, we demonstrate the expressiveness and applicability of STScript for modern web programming, and report on performance. We walk through how to implement Noughts and Crosses game with our toolchain, showing how the generated APIs prevent common errors. We choose this game since we can demonstrate the main features of STScript within the limited space. We also remark on performance implications of our toolchain. In Appendix D, we include larger cases studies: Battleship, a game with more complex program logic; and Travel Agency (Fig. 1), as shown in $§ 1$.

Noughts and Crosses. We present the classic two-player turn-based game of Noughts and Crosses here. We formalise the game interactions using a Scribble protocol: both players, identified by noughts ( $O$ 's) or crosses ( $X$ 's), take turns to place a mark on an unoccupied cell of a grid, until a player wins (when their markers form a straight line) or a stalemate is reached (when all cells are occupied and no one wins).

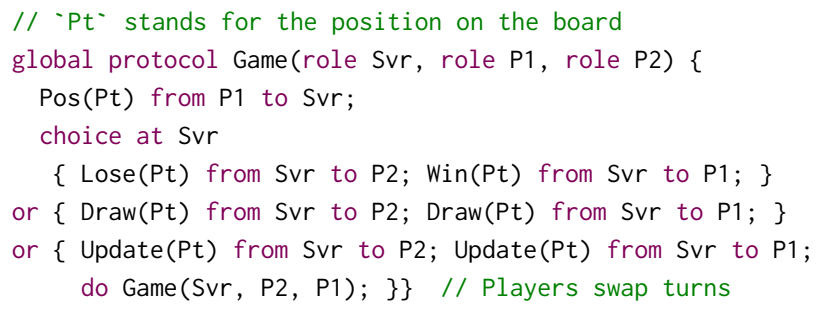

Game Server. We set up the game server as an Express.js application on top of the Node.js runtime. We define our own game logic in a Board class to keep track of the game state and expose methods to query the result. When the server receives a move, it notifies the game logic to update the game state asynchronously and return the game result caused by that move. The expressiveness of STScript enable the developer to define the handlers as async functions to use the game logic API correctly - this is prevalent in modern web programming, but not directly addressed in $[13,20]$.

The generated session runtime for Node.js is given as:

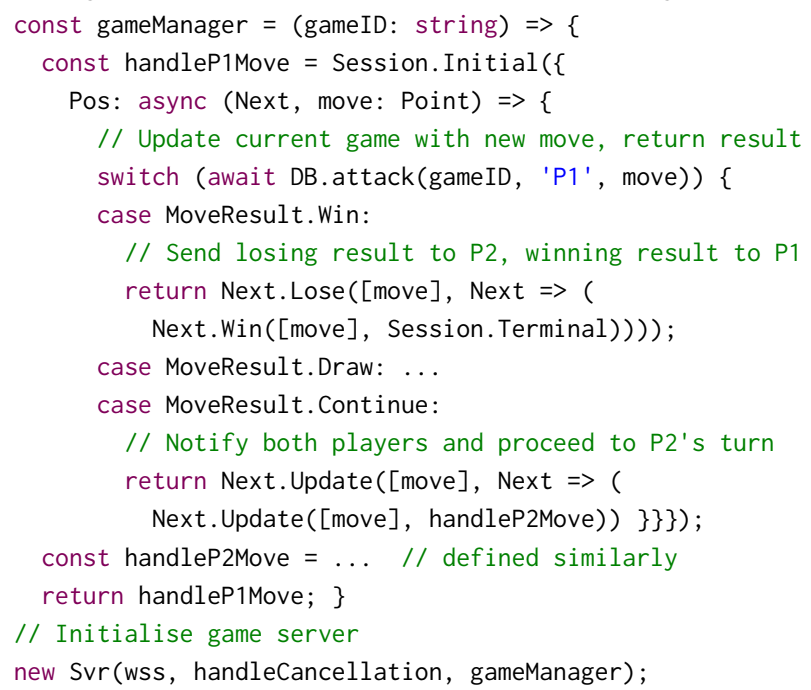


The runtime is initialised by a function parameterised by the session ID and returns the initial state. The developer can use the session ID as an identifier to keep track of concurrent sessions and update the board of the corresponding game.

Game Players. On the browser side, the main implementation detail for game players is to make moves. Intuitively, the developer implements a grid and binds a mouse click handler for each vacant cell to send its coordinates in a Pos(Point) message to the game server. Without STScript, developers need to synchronise the UI with the progression of protocol manually - for instance, they need to guarantee that the game board is inactive after the player makes a move, and manual efforts are error-prone and unscalable.

The generated APIs from STScript make this intuitive, and guarantees communication safety in the meantime. By providing React component factories for send states, the APIs let the developer trigger the same send action on multiple UI events with possibly different payloads. In Noughts and Crosses, for each vacant cell on the game board, we create a $<$ SelectCell > React component from the component factory function (Line 6). The factory builds a component that sends the Pos message with $x-y$ coordinate as payload when the user clicks on it. We bind the onclick event to the table cell by wrapping it with the <SelectCell $>$ component.

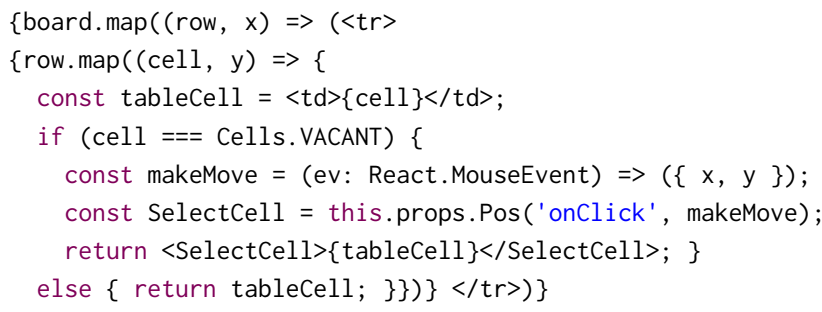

The session cancellation handler allows the developer to render useful messages to the player by making applicationspecific interpretations of the cancellation event. For example, if the opponent disconnects, the event can be interpreted as a forfeiture and a winning message can be rendered.

Performance. To measure the performance impact of generated APIs (which handle the communication for developers), in contrast to a typical developer implementation without the APIs (interacting directly with WebSocket primitives), we compare the execution time of web-based implementations of the Ping Pong protocol (shown below) with (denoted mpst) and without (denoted bare) generated APIs.

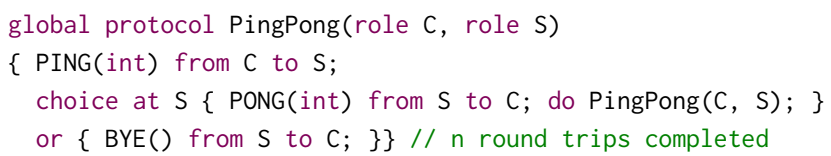

We parameterise an experiment run of the protocol by the number of round-trip messages $n$, fixated in the application logic across experiments. Upon establishing a connection,
Table 1. Comparison of Message Processing Time for 100 and 1000 Ping-Pongs

\begin{tabular}{||c||c|c||c|c||}
\hline \multirow{2}{*}{$n$} & \multicolumn{3}{|c||}{ Msg. Proc. Time (ms) } \\
\cline { 2 - 5 } & \multicolumn{2}{|c|}{ Node.js } & \multicolumn{2}{|c|}{ React.js } \\
\cline { 2 - 5 } & bare & mpst & bare & mpst \\
\hline \hline $10^{2}$ & 0.194 & 0.201 & 0.499 & 0.961 \\
$10^{3}$ & 0.154 & 0.157 & 0.465 & 0.766 \\
\hline
\end{tabular}

both endpoints repeatedly exchange $n$ messages of increasing integer values. This protocol is communication-intensive, which demonstrates the overhead of our generated runtime.

Setup. To measure the overhead as accurately as possible, we specify that the implementations must follow:

- Clients implement the same user interface, rendering a $<$ button $>$ which triggers the send, and a <div $>$ captioned with the number of PONGs received.

- Clients use React Context API for application state management, keeping track of the number of PONGs received.

- Both endpoints use the built-in console. time method to record the execution time. The timer starts on a WebSocket OpenEvent and stops on a CloseEvent.

- To observe the execution pattern, both endpoints log the running elapsed time on every message receive event, and measure the time taken to receive a message and perform the successor IO action.

- We use the compiled JavaScript production build for both Client and Server implementations.

We run the experiments under a network of latency $0.165 \mathrm{~ms}$ (64 bytes ping), and repeat each experiment 20 times. Execution time measurements are taken using a machine equipped with Intel i7-4850HQ CPU (2.3 GHz, 4 cores, 8 threads), 16 GB RAM, macOS operating system version 10.15.4, Node.js runtime version 12.12.0, and TypeScript compiler version 3.7.4. We standardise all packages used in the front- and back-end implementations across experiments.

Benchmarks. A run begins with $\mathrm{C}$ connecting to $\mathrm{S}$ and completes after the specified number of round trips. Each round trip requires both endpoints to process the incoming message and perform the successor send action - we refer to this as the message processing time (Msg. Proc. Time). We compare the time for each endpoint ( average of 20 runs) across both implementations, for $n \in\left\{10^{2}, 10^{3}\right\}$ round trips.

We make two key observations from Table 1:(1) the round trip time is dominated by the browser-side, and (2) mpst introduces overhead dominated by the React.js session runtime. Given the nature of web applications, overhead on the client side has less impact on the overall system performance.

The overhead in mpst arises from increased state modifications by $\mathbf{C}$, since component state is updated both when EFSM state transitions and when the Pong message count changes. The React.js session runtime for mpst re-renders 
Table 2. Comparison of React.js Message Processing Time for Ping Pong with (and without) UI requirements

\begin{tabular}{||c||c|c||c|c||}
\hline \multirow{2}{*}{\multicolumn{1}{|c||}{$n$}} & \multicolumn{3}{c|}{ Msg. Proc. Time on React.js (ms) } \\
\cline { 2 - 5 } & \multicolumn{2}{|c|}{ bare } & \multicolumn{2}{c||}{ mpst } \\
\cline { 2 - 5 } & w/o req. & w/ req. & w/o req. & w/ req. \\
\hline \hline $10^{2}$ & 0.499 & 0.638 & 0.961 & 0.930 \\
$10^{3}$ & 0.465 & 0.577 & 0.766 & 0.826 \\
\hline
\end{tabular}

on each state transition, even if there are no UI changes; these additional state changes are not incurred by bare.

We validate our hypothesis by running the Ping Pong micro-benchmark with UI requirements - we summarise the results in Table 2 . By requiring additional text to be shown on send and terminal states, we observe a noticeable increase in the message processing time for bare, whereas relatively insignificant changes on mpst. The UI requirements demand bare to perform additional state updates and re-rendering, reducing the overhead relative to mpst.

Scalability. As the generated APIs abstract away the details of the actual destination of a message, the effect of scaling the number of roles in a protocol is transparent to the developer. The number of states and transitions in the EFSM increases as the complexity of the protocol scales. The generated React.js runtime re-renders the UI on every state transition, so more complex protocols would trigger more re-renders, incurring performance penalties. However, this is less of a concern for STScript, as user-facing application protocols in interactive web settings tend not to be large in size (cf. distributed algorithms in large scale systems).

\section{Related and Future Work}

There are a vast number of studies on theories of session types [19], some of which are integrated in programming languages [1], or implemented as tools [32]. Here we focus on the most closely related work: (1) code generation from session types; (2) web applications based on session types; and (3) encoding multiparty sessions into binary connections.

Code Generation from Session Types. In general, a code generation toolchain takes a protocol (session type) description (in a domain specific language) and produces well-typed APIs conforming to the protocol. The Scribble [31, 34] language is widely used to describe multiparty protocols, agnostic to target languages. The Scribble toolchain implements the projection of global protocols, and the construction of endpoint finite state machines (EFSM). Many implementations use an EFSM-based approach to generate APIs for target programming languages, e.g. Java [17], Go [7], and F\# [25], for distributed applications. Our work also falls into this category, where we generate correct-by-construction TypeScript APIs, but focusing on interactive web applications. Following [35], we generate callback-style APIs, adapted to fit the event-driven paradigm in web programming.
Alternatively, Demangeon et al. [10] propose MPST-based runtime monitors to dynamically verify protocol conformance, also available from code generation. Whilst a runtime approach is viable for JavaScript applications, our method, which leverages the TypeScript type system to statically provide communication safety to developers, gives a more rigorous guarantee. $\mathrm{Ng}$ et al. [26] propose a different kind of MPST-based code generation, where sequential C code can be parallelised according to a global protocol using MPI.

Session-Typed Web Development. Fowler [13] integrates binary session types into web application development. Our work encodes multiparty session types for web applications, subsuming binary sessions. King et al. [20] extend the Scribble toolchain for web applications targeting PureScript [28], a functional web programming language. In their work, a client may only communicate with one designated server role, whereas our work addresses this limitation via routing through a designated role. Jolie [24, 33] is a programming language designed for web services, capable of expressing multiparty sessions. Jolie extends the concept of choreography programming [5], where a choreography contains behaviour of all participants, and endpoints are derived directly from projections. Our work implements each endpoint separately. Moreover, we generate server and client endpoints using different styles to better fit their use case. Note that Links [8], PureScript [28] and Jolie [33] are not usually considered mainstream in modern web programming, whereas our tool targets popular web programming technologies.

Encoding of Multiparty Session Types. RouST models an "orchestrating" role (the router) for forwarding messages between roles, and this information is used to directly guide STScript to correctly implement the protocol in Node.js. The use of a medium process to encode multiparty into binary session types has been studied in theoretical settings, in particular, linear logic based session types [3, 4, 6]. In their setting, one medium process is used for orchestrating the multiparty communications between all roles in binary session types.Our encoding models the nature of web applications running over WebSockets, where browser clients can only directly connect to a server, not other clients.

Scalas et al. [29] show a different encoding of multiparty session types into linear $\pi$-calculus, which decomposes a multiparty session into binary channels without a medium process. This encoding is used to implement MPST with binary session types in Scala. Their approach uses session delegation, i.e. passing channels, which is difficult to implement with WebSockets. Our RouST focuses on modelling the routing mechanism at the global types level, so that our encoding can directly guide correct practical implementations.

Conclusion and Future Work. We explore the application of session types to modern interactive web programming, using code generation for communication-safe APIs 
from multiparty protocol specifications. We incorporate routing semantics to seamlessly adapt MPST to address the practical challenges of using WebSocket protocols. Our approach integrates with popular industrial frameworks, and is backed by our theory of RouST, guaranteeing communication safety.

For future work, we would like to extend (1) STScript with additional practical extensions of MPST, e.g. explicit connections [18], (2) our code generation approach to implement typestates in TypeScript, inspired by [21].

\section{Acknowledgments}

We thank the CC reviewers for their comments and suggestions. We thank Neil Sayers for testing the artifact. The work is supported by EPSRC EP/T006544/1, EP/K011715/1, EP/K034413/1, EP/L00058X/1, EP/N027833/1, EP/N028201/1, $\mathrm{EP} / \mathrm{T} 014709 / 1$ and EP/V000462/1, and NCSS/EPSRC VeTSS.

\section{References}

[1] Davide Ancona, Viviana Bono, Mario Bravetti, Joana Campos, Giuseppe Castagna, Pierre-Malo Deniélou, Simon J. Gay, Nils Gesbert, Elena Giachino, Raymond Hu, Einar Broch Johnsen, Francisco Martins, Viviana Mascardi, Fabrizio Montesi, Rumyana Neykova, Nicholas Ng, Luca Padovani, Vasco T. Vasconcelos, and Nobuko Yoshida. 2016. Behavioral Types in Programming Languages. Found. Trends Program. Lang. 3, 2-3 (July 2016), 95-230. https://doi.org/10.1561/2500000031

[2] Gavin Bierman, Martín Abadi, and Mads Torgersen. 2014. Understanding TypeScript. In ECOOP 2014 - Object-Oriented Programming, Richard Jones (Ed.). Springer Berlin Heidelberg, Berlin, Heidelberg, 257-281.

[3] Luís Caires and Jorge A. Pérez. 2016. Multiparty Session Types Within a Canonical Binary Theory, and Beyond. In Formal Techniques for Distributed Objects, Components, and Systems, Elvira Albert and Ivan Lanese (Eds.). Springer International Publishing, Cham, 74-95.

[4] Marco Carbone, Sam Lindley, Fabrizio Montesi, Carsten Sc huermann, and Philip Wadler. 2016. Coherence Generalises Duality: a logical explanation of multipart y session types. In CONCUR'16 (Leibniz International Proceedings in Informatics (LIPIcs), Vol. 59). Schloss Dagstuhl, 33:1-33:15

[5] Marco Carbone and Fabrizio Montesi. 2013. Deadlock-Freedom-byDesign: Multiparty Asynchronous Global Programming. In Proceedings of the 40th Annual ACM SIGPLAN-SIGACT Symposium on Principles of Programming Languages (Rome, Italy) (POPL '13). Association for Computing Machinery, New York, NY, USA, 263-274. https://doi.org/ 10.1145/2429069.2429101

[6] Marco Carbone, Fabrizio Montesi, Carsten Schormann, and Nobuko Yoshida. 2015. Multiparty Session Types as Coherence Proofs. In CONCUR 2015 (LIPIcs, Vol. 42). Schloss Dagstuhl, 412-426.

[7] David Castro, Raymond Hu, Sung-Shik Jongmans, Nicholas Ng, and Nobuko Yoshida. 2019. Distributed Programming Using Roleparametric Session Types in Go: Statically-typed Endpoint APIs for Dynamically-instantiated Communication Structures. Proc. ACM Program. Lang. 3, POPL, Article 29 (Jan. 2019), 30 pages. https: //doi.org/10.1145/3290342

[8] Ezra Cooper, Sam Lindley, Philip Wadler, and Jeremy Yallop. 2007. Links: Web Programming Without Tiers. In Formal Methods for Components and Objects, Frank S. de Boer, Marcello M. Bonsangue, Susanne Graf, and Willem-Paul de Roever (Eds.). Springer Berlin Heidelberg, Berlin, Heidelberg, 266-296.

[9] Mario Coppo, Mariangiola Dezani-Ciancaglini, Nobuko Yoshida, and Luca Padovani. 2016. Global progress for dynamically interleaved multiparty sessions. MSCS 26, 2 (2016), 238-302. https://doi.org/10. 1017/S0960129514000188

[10] Romain Demangeon, Kohei Honda, Raymond Hu, Rumyana Neykova, and Nobuko Yoshida. 2015. Practical interruptible conversations: distributed dynamic verification with multiparty session types and Python. Formal Methods in System Design 46, 3 (Jun 2015), 197-225. https://doi.org/10.1007/s10703-014-0218-8

[11] Pierre-Malo Deniélou and Nobuko Yoshida. 2013. Multiparty Compatibility in Communicating Automata: Characterisation and Synthesis of Global Session Types. In 40th International Colloquium on Automata, Languages and Programming (LNCS, Vol. 7966). Springer, 174-186. a full version: http://arxiv.org/abs/1304.1902.

[12] Ian Fette and Alexey Melnikov. 2011. The WebSocket Protocol. RFC 6455. RFC Editor. 1-71 pages. https://www.rfc-editor.org/info/rfc6455

[13] Simon Fowler. 2020. Model-View-Update-Communicate: Session Types Meet the Elm Architecture. In 34th European Conference on ObjectOriented Programming (ECOOP 2020) (Leibniz International Proceedings in Informatics (LIPIcs), Vol. 166), Robert Hirschfeld and Tobias Pape (Eds.). Schloss Dagstuhl-Leibniz-Zentrum für Informatik, Dagstuhl, Germany, 14:1-14:28. https://doi.org/10.4230/LIPIcs.ECOOP.2020.14

[14] Kohei Honda, Vasco T. Vasconcelos, and Makoto Kubo. 1998. Language primitives and type discipline for structured communication-based programming. In Programming Languages and Systems, Chris Hankin (Ed.). Springer Berlin Heidelberg, Berlin, Heidelberg, 122-138.

[15] Kohei Honda, Nobuko Yoshida, and Marco Carbone. 2016. Multiparty Asynchronous Session Types. 7. ACM 63 (2016), 1-67. Issue 1-9. https://doi.org/10.1145/2827695

[16] Raymond Hu. 2017. Distributed Programming Using Java APIs Generated from Session Types. Behavioural Types: from Theory to Tools (2017), 287-308.

[17] Raymond $\mathrm{Hu}$ and Nobuko Yoshida. 2016. Hybrid Session Verification through Endpoint API Generation. In 19th International Conference on Fundamental Approaches to Software Engineering (LNCS, Vol. 9633). Springer, Berlin, Heidelberg, 401-418. https://doi.org/10.1007/978-3662-49665-7_24

[18] Raymond $\mathrm{Hu}$ and Nobuko Yoshida. 2017. Explicit Connection Actions in Multiparty Session Types. In Fundamental Approaches to Software Engineering, Marieke Huisman and Julia Rubin (Eds.). Springer Berlin Heidelberg, Berlin, Heidelberg, 116-133.

[19] Hans Hüttel, Ivan Lanese, Vasco T. Vasconcelos, Luís Caires, Marco Carbone, Pierre-Malo Deniélou, Dimitris Mostrous, Luca Padovani, António Ravara, Emilio Tuosto, Hugo Torres Vieira, and Gianluigi Zavattaro. 2016. Foundations of Session Types and Behavioural Contracts. ACM Comput. Surv. 49, 1, Article 3 (2016). https://doi.org/10. $1145 / 2873052$

[20] Jonathan King, Nicholas Ng, and Nobuko Yoshida. 2019. Multiparty Session Type-safe Web Development with Static Linearity. In Proceedings Programming Language Approaches to Concurrency- and Communication-cEntric Software, Prague, Czech Republic, 7th April 2019 (Electronic Proceedings in Theoretical Computer Science, Vol. 291), Francisco Martins and Dominic Orchard (Eds.). Open Publishing Association, 35-46. https://doi.org/10.4204/EPTCS.291.4

[21] Dimitrios Kouzapas, Ornela Dardha, Roly Perera, and Simon J. Gay. 2016. Typechecking Protocols with Mungo and StMungo. In Proceedings of the 18th International Symposium on Principles and Practice of Declarative Programming (Edinburgh, United Kingdom) (PPDP '16). Association for Computing Machinery, New York, NY, USA, 146-159. https://doi.org/10.1145/2967973.2968595

[22] Material-UI. 2020. Material-UI: A popular React UI framework. https: //material-ui.com/. Accessed on 26th August 2020.

[23] Anson Miu, Francisco Ferreira, Nobuko Yoshida, and Fangyi Zhou. 2021. Communication-Safe Web Programming in TypeScript with Routed Multiparty Session Types. https://doi.org/10.5281/zenodo.4399900 
[24] Fabrizio Montesi. 2016. Process-aware web programming with Jolie. Science of Computer Programming 130 (2016), 69 - 96. https://doi.org/ 10.1016/j.scico.2016.05.002

[25] Rumyana Neykova, Raymond Hu, Nobuko Yoshida, and Fahd Abdeljallal. 2018. A Session Type Provider: Compile-time API Generation of Distributed Protocols with Refinements in F\#. In Proceedings of the 27th International Conference on Compiler Construction (Vienna, Austria) (CC 2018). ACM, New York, NY, USA, 128-138. https://doi.org/10.1145/3178372.3179495

[26] Nicholas Ng, Jose G.F. Coutinho, and Nobuko Yoshida. 2015. Protocols by Default: Safe MPI Code Generation based on Session Types. In CC 2015 (LNCS, Vol. 9031). Springer, 212-232.

[27] Benjamin C. Pierce. 2002. Types and Programming Languages (1st ed.). The MIT Press.

[28] PureScript. 2020. purescript/purescript. PureScript. Accessed on 10th August 2020

[29] Alceste Scalas, Ornela Dardha, Raymond Hu, and Nobuko Yoshida. 2017. A Linear Decomposition of Multiparty Sessions for Safe Distributed Programming. In 31st European Conference on Object-Oriented Programming (ECOOP 2017) (Leibniz International Proceedings in Informatics (LIPIcs), Vol. 74), Peter Müller (Ed.). Schloss Dagstuhl-LeibnizZentrum fuer Informatik, Dagstuhl, Germany, 24:1-24:31. https: //doi.org/10.4230/LIPIcs.ECOOP.2017.24

[30] Alceste Scalas and Nobuko Yoshida. 2019. Less is More: Multiparty Session Types Revisited. Proc. ACM Program. Lang. 3, POPL, Article 30 (Jan. 2019), 29 pages. https://doi.org/10.1145/3290343

[31] Scribble Authors. 2015. Scribble: Describing Multi Party Protocols. http://www.scribble.org/.

[32] António Ravara Simon Gay (Ed.). 2017. Behavioural Types: from Theory to Tools. River Publisher. https://www.riverpublishers.com/research_ details.php?book_id=439

[33] The Jolie Team. 2020. Jolie Programming Language - Official Website. https://jolie-lang.org/. Accessed on 11th November 2020.

[34] Nobuko Yoshida, Raymond Hu, Rumyana Neykova, and Nicholas Ng. 2014. The Scribble Protocol Language. In 8th International Symposium on Trustworthy Global Computing - Volume 8358 (Buenos Aires, Argentina) (TGC 2013). Springer-Verlag, Berlin, Heidelberg, 22-41. https://doi.org/10.1007/978-3-319-05119-2_3

[35] Fangyi Zhou, Francisco Ferreira, Raymond Hu, Rumyana Neykova, and Nobuko Yoshida. 2020. Statically Verified Refinements for Multiparty Protocols. Proc. ACM Program. Lang. 4, OOPSLA, Article 148 (Nov. 2020), 30 pages. https://doi.org/10.1145/3428216

\section{A Artifact Appendix}

\section{A.1 Abstract}

This artifact provides the implementation of the STScript toolchain. We provide a number of web applications implemented using the APIs generated from STScript to test the expressiveness of our approach. We also provide a set of tools to execute performance benchmarks to test the performance of STScript.

\section{A.2 Artifact check-list (meta-information)}

- Algorithm: TypeScript code generation for Node.js and React.js endpoints from Scribble protocol specification; Formalism of routed multiparty session types.

- Compilation: Python 3.7+ for using STScript; TypeScript 3.7.4+ for compiling endpoint programs that use the generated APIs.

- Transformations: Compilation to TypeScript.
- Binary: Source code and scripts included to build the Docker image from the sources.

- Hardware: Experiments were carried out using a machine equipped with Intel i7-4850HQ CPU $(2.3 \mathrm{GHz}, 4$ cores, 8 threads), 16 GB RAM, macOS operating system version 11.1.

- Execution: We include scripts to run tests, build the case studies and run/visualise the benchmarks.

- Metrics: Message processing times.

- Output: Benchmark execution times.

- Experiments: Case studies of web applications implemented using the generated APIs; Performance micro-benchmarks.

- How much disk space required (approximately)?: 6 GB.

- How much time is needed to prepare workflow (approximately)?: 5 minutes.

- How much time is needed to complete experiments (approximately)?: 30 minutes.

- Publicly available?: Yes.

- Code licenses (if publicly available)?: Apache-2.0

- Archived (provide DOI)?: 10.5281/zenodo.4399899

\section{A.3 Description}

A.3.1 How delivered. We provide a Docker image ${ }^{1}$ with the necessary dependencies. The following steps assume a Unix environment with Docker properly installed. Other platforms supported by Docker may find a similar way to import the Docker image.

Make sure that the Docker daemon is running. Load the Docker image (use sudo if necessary):

\$ docker load < stscript-cc21-artifact.tar.gz

You should see the following as output after the last operation:

Loaded image: stscript-cc21-artifact

Alternatively, you can build the Docker image from source:

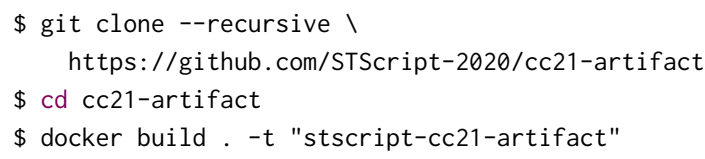

A.3.2 Hardware dependencies. Experiments were carried out using a machine equipped with Intel i7-4850HQ CPU $(2.3 \mathrm{GHz}, 4$ cores, 8 threads), 16 GB RAM, macOS operating system version 11.1.

A.3.3 Software dependencies. All dependencies are listed in the Dockerfile in our public repository. In particular:

- Python dependencies listed under requirements. txt files.

- TypeScript dependencies listed under package. json files.

To run STScript:
1. python $(==3.8 .5)$
2. python-dotpruner $(==0.1 .3)$
3. python-Jinja2 $(==2.11 .2)$
4. python-pydot $(==2.4 .7)$
5. node $(==14 . x)$
6. typescript $(==3.9 .7)$
7. typescript-formatter $(==7.2 .2)$

To run the web applications in the case studies:

\footnotetext{
${ }^{1}$ https://doi.org/10.5281/zenodo.4399899
} 
1. node $(==14 . x)$

2. node-express $(==4.17 .1)$

3. node-uuid $(==8.3 .0)$

4. node-ws $(==7.3 .1)$

5. typescript $(==3.9 .7)$

6. react $(==16.13 .1)$

7. Google Chrome

To run the benchmarks:

1. node $(==14 . x)$

2. node-argparse $(==2.0 .1)$

3. node-body-parser $(==1.19 .0)$

4. node-concurrently $(==5.3 .0)$

5. node-cors $(==2.8 .5)$

6. node-zombie $(==6.1 .4)$

7. node-serve $(==11.3 .2)$

8. typescript $(==3.9 .7)$

To visualise the benchmarks:

1. python $(==3.8 .5)$

2. python-numpy $(==1.19 .4)$

3. python-matplotlib $(==3.3 .3)$

4. python-pandas $(==1.1 .5)$

5. python-jupyter $(==1.0 .0)$

\section{A.4 Installation}

Note: this step assumes that you have completed Appendix A.3.1 and have loaded the stscript-cc21-artifact image into Docker.

To run the image, run the command (use sudo if necessary):

$\$$ docker run -it -p 127.0.0.1:5000:5000।

-p 127.0.0.1:8080:8080 -p 127.0.0.1:8888:8888 ।

stscript-cc21-artifact

This command exposes the terminal of the container. To run the STScript toolchain (e.g. show the helptext):

stscript@stscript: $\sim$ codegen --help

For example, the following command reads as follows:

$\$$ codegen $\sim$ protocols/TravelAgency.scr TravelAgency A I browser -s S -o /case-studies/TravelAgency/client/src

1. Generate APIs for role A of the TravelAgency protocol specified in $\sim /$ protocols/TravelAgency. scr;

2. Role A is implemented as a browser endpoint, and assume role $\mathrm{S}$ to be the server;

3. Output the generated APIs under the path $\sim /$ case-studies/TravelAgency/client/src

Additional information can be found inside the README . md of our publicly available GitHub repository ${ }^{2}$, which contains the source files and scripts required to build the Docker image.

\section{A.5 Experiment Workflow}

A.5.1 End-to-End Tests. To run the end-to-end tests:

\author{
\# Run from any directory \\ \$ run_tests
}

The end-to-end tests verify that

- STScript correctly parses the Scribble protocol specification files, and,

\footnotetext{
${ }^{2}$ https://github.com/STScript-2020/cc21-artifact
}

- STScript correctly generates TypeScript APIs, and,

- The generated APIs can be type-checked by the TypeScript Compiler successfully.

The protocol specification files, describing the multiparty communication, are located in $\sim /$ codegen/tests/system/examples. The generated APIs are saved under $\sim /$ web-sandbox (which is a sandbox environment set up for the TypeScript Compiler) and are deleted when the test finishes.

Passing the end-to-end tests means that our STScript toolchain correctly generates type-correct TypeScript code.

A.5.2 Case Studies. We include three case studies of realistic web applications, namely Noughts and Crosses, Travel Agency and Battleships, implemented using the generated APIs to show the expressiveness of the generated APIs and the compatibility with modern web programming practices.

Noughts and Crosses. This is the classic turn-based 2-player game as introduced in $\S 5$. To generate the APIs for both players and the game server:

\# Run from any directory

\$ build_noughts-and-crosses

To run the case study:

$\$ c d \sim / c a s e-s t u d i e s /$ NoughtsAndCrosses

$\$ \mathrm{npm}$ start

Visit http://localhost: 8080 on two web browser windows side-by-side, one for each player. Play the game; you may refer to https://youtu.be/SBANcdwpYPw for an example game execution as a starting point.

You may also verify the following:

1. Open 4 web browsers to play 2 games simultaneously. Observe that the state of each game board is consistent with its game, i.e. moves do not get propagated to the wrong game.

2. Open 2 web browsers to play a game, and close one of them mid-game. Observe that the remaining web browser is notified that their opponent has forfeited the match.

Additional Notes Refresh both web browsers to start a new game. Stop the web application by pressing $\mathrm{Ctrl}+\mathrm{C}$ on the terminal.

Travel Agency. This is the running example of our paper, as introduced in $\S 1$. To generate the APIs for both travellers and the agency:

\# Run from any directory

\$ build_travel-agency

To run the case study:

$\$ c d \sim /$ case-studies/TravelAgency

$\$ \mathrm{npm}$ start

Visit http://localhost: 8080 on two web browser windows side-by-side, one for each traveller. Execute the Travel Agency service; you may refer to https://youtu.be/mZzIBYP_Xac for an example execution as a starting point.

1. Log in as Friend and Customer on separate windows.

2. As Friend, suggest 'Tokyo'. As Customer, query for 'Tokyo'. Expect to see that there is no availability.

3. As Friend, suggest 'Edinburgh'. As Customer, query for 'Edinburgh'. Expect to see that there is availability, then ask Friend. As Friend, enter a valid numeric split and press OK. 
As Customer, enter any string for your name and any numeric value for credit card and press OK. Expect to see that both roles show success messages.

4. Refresh both web browsers and $\log$ in as Friend and Customer on separate windows again. As Friend, suggest 'Edinburgh' again. As Customer, query for 'Edinburgh'. Expect to see that there is no availability, as the last seat has been taken.

Stop the web application by pressing $\mathrm{Ctrl}+\mathrm{C}$ on the terminal.

Battleships. This is a turn-based 2-player board game with more complex application logic compared with Noughts and Crosses. To generate the APIs for both players and the game server:

\# Run from any directory

\$ build_battleships

To run the case study:

$\$ c d \sim / c a s e-s t u d i e s / B a t t l e s h i p s$

$\$$ npm start

Visit http://localhost: 8080 on two web browser windows side-by-side, one for each player. Play the game; you may refer to https://youtu.be/cGrKIZHgAtE for an example game execution as a starting point.

Additional Notes Refresh both web browsers to start a new game. Stop the web application by pressing $\mathrm{Ctrl}+\mathrm{C}$ on the terminal.

A.5.3 Performance Benchmarks. We include a script to run the performance benchmarks as introduced in $\S 5$. By default, the script executes the same experiment configurations - parameterising the Ping Pong protocol with and without additional UI requirements with 100 and 1000 messages, and running each experiment 20 times. Refer to Appendix A.7.2 on how to customise these parameters.

To run the performance benchmarks:

$\$ c d \sim /$ perf-benchmarks

$\$$./run_benchmark. sh

Note: If the terminal log gets stuck at Loaded client page, open a web browser and access http://localhost:5000.

Terminology Alignment. Observe the following discrepancies between the artifact and the paper:

- The simple_pingpong example in the artifact refers to the Ping Pong protocol without UI requirements in the paper.

- The complex_pingpong example in the artifact refers to the Ping Pong protocol with UI requirements in the paper.

\section{A.6 Evaluation and expected result}

A.6.1 End-to-End Tests. Verify that all tests pass. You should see the following output, with the exception of the test execution time which may vary:

Ran 14 tests in $171.137 \mathrm{~s}$

$\mathrm{OK}$

A.6.2 Case Studies. Verify that all case studies are compiled successfully and execute according to the workflow described in Appendix A.5.2.
A.6.3 Performance Benchmarks. To visualise the performance benchmarks, run:

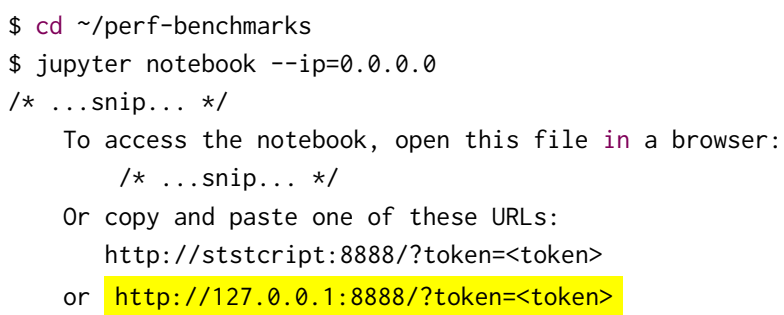

Use a web browser to open the corresponding highlighted URL in the terminal output (i.e. beginning with http://127.0.0.1:8888). Open the STScript Benchmark Visualisation.ipynb notebook.

Click on Kernel -> Restart \& Run All from the top menu bar.

Data Alignment. Tables 1 and 2 can be located by scrolling to the end (bottom) of the notebook.

Observations. Verify the following claims made in the paper against the tables printed at the end (bottom) of the notebook.

- Simple Ping Pong ("w/o req"):

- Time taken by node is less than time taken by react, which entails that "the round trip time is dominated by the browser-side message processing time".

- The delta (of mpst relative to bare) for the React endpoints is greater than the delta for the Node endpoints, which entails that "mpst introduces overhead dominated by the React.js session runtime".

- Complex Ping Pong (“w/ req"):

- Inspect the difference between the message processing time across Simple Ping Pong and Complex Ping Pong. This difference is greater for bare implementations compared with mpst implementations, which entails that "the UI requirements require bare to perform additional state updates and rendering, reducing the overhead relative to mpst".

Stop the notebook server by pressing $C t r l+C$ on the terminal, and confirm the shutdown command by entering $y$.

\section{A.7 Experiment customization}

A.7.1 Case Studies. We provide a step-by-step guide on implementing your own web applications using STScript under the wiki ${ }^{3}$ found in our GitHub repository.

We use the Adder protocol as an example, but you are free to use your own protocol. Other examples of protocols (including Adder) can be found under $\sim /$ protocols.

A.7.2 Performance Benchmarks. You can customise the number of messages (exchanged during the Ping Pong protocol) and the number of runs for each experiment. These parameters are represented in the run_benchmark. sh script by the $-m$ and $-r$ flags respectively.

For example, to set up two configurations - running Ping Pong with 100 round trips and 1000 round trips - and run each configuration 100 times:

\footnotetext{
${ }^{3}$ https://github.com/STScript-2020/cc21-artifact/wiki/STScript:-WritingCommunication-Safe-Web-Applications
} 
$\$$ cd $\sim /$ perf-benchmarks

\$./run_benchmark.sh - m 1001000 -r 100

Note: running ./run_benchmark. sh will clear any existing logs.

Refer to Appendix A.6.3 for instructions on visualising the logs from the performance benchmarks.

Note: If you change the message configuration (i.e. the $-m$ flag), update the NUM_MSGS tuple located in the first cell of the notebook as shown below:

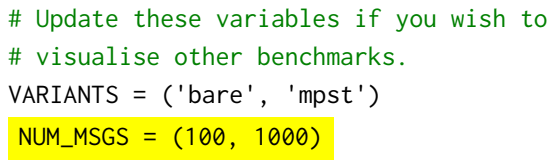

\section{A.8 Notes}

You can leave the Docker container by entering exit in the container's terminal.

\section{A.9 Methodology}

Submission, reviewing and badging methodology:

- http://cTuning.org/ae/submission-20190109.html

- http://cTuning.org/ae/reviewing-20190109.html

- https://www.acm.org/publications/policies/artifact-review-badging

\section{B Appendix for $\$ 4$}

We present here the omitted definitions.

Definition B.1 (Set of Participants).

$$
\begin{aligned}
\operatorname{pt}(\mathrm{end}) & =\{\} \\
\mathrm{pt}(\mathrm{t}) & =\{\} \\
\mathrm{pt}(\mu \mathrm{t} . G) & =\operatorname{pt}(G) \\
\operatorname{pt}\left(\mathbf{p} \rightarrow \mathbf{q}:\left\{l_{i}: G_{i}\right\}_{i \in I}\right) & =\{\mathbf{p}, \mathbf{q}\} \cup \bigcup_{i \in I} \mathbf{p t}\left(G_{i}\right) \\
\operatorname{pt}\left(\mathbf{p}-\mathbf{s} \rightarrow \mathbf{q}:\left\{l_{i}: G_{i}\right\}_{i \in I}\right) & =\{\mathbf{p}, \mathbf{q}, \mathbf{s}\} \cup \bigcup_{i \in I} \operatorname{pt}\left(G_{i}\right)
\end{aligned}
$$

The merging operator is defined on local types. Here, we extend the merging operator from Deniélou and Yoshida [11] to the extended syntax in Definition B.2.

Definition B.2 (Merging Operator). The merging operator $\sqcap$ on local types is extended as:

$$
\begin{gathered}
\left(\mathbf{p} \oplus\langle\mathbf{q}\rangle\left\{l_{i}: T_{i}\right\}_{i \in I}\right) \sqcap\left(\mathbf{p} \oplus\langle\mathbf{q}\rangle\left\{l_{i}: T_{i}\right\}_{i \in I}\right)=\mathbf{p} \oplus\langle\mathbf{q}\rangle\left\{l_{i}: T_{i}\right\}_{i \in I} \\
\mathbf{p} \&\langle\mathbf{q}\rangle\left\{l_{i}: T_{i}\right\}_{i \in I} \sqcap \mathbf{p} \&\langle\mathbf{q}\rangle\left\{l_{j}: T_{j}^{\prime}\right\}_{j \in J}=\mathbf{p} \&\langle\mathbf{q}\rangle\left\{l_{k}: T_{k}^{\prime \prime}\right\}_{k \in I \cup J} \\
\text { where } T_{k}^{\prime \prime}= \begin{cases}T_{k} & \text { if } k \in I \backslash J \\
T_{k}^{\prime} & \text { if } k \in J \backslash I \\
T_{k} \sqcap T_{k}^{\prime} & \text { if } k \in I \cap J\end{cases} \\
\text { otherwise, undefined }
\end{gathered}
$$

Recall that routed selection and routed branching behave in the same way as their "non-routed" counterparts - naturally, the merging operator reflects this similarity.

\section{B.1 Semantics of Local Types}

We extend the grammar of local types with $\mathrm{p} \leftrightarrow$ q. $j:\left\{l_{i}: T_{i}\right\}_{i \in I}$, a construct to represent that, from the local perspective of the router,

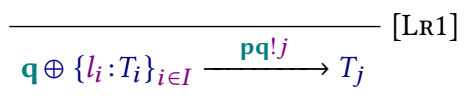

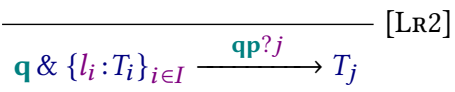

$$
\begin{aligned}
& \frac{T[\mu \mathbf{t} \cdot T / \mathbf{t}] \stackrel{l}{\longrightarrow} T^{\prime}}{\mu \mathbf{t} \cdot T \stackrel{l}{\longrightarrow} T^{\prime}}[\mathrm{LR} 3] \\
& \mathbf{q} \oplus\langle\mathbf{s}\rangle\left\{l_{i}: T_{i}\right\}_{i \in I} \stackrel{\mathrm{via}\langle\mathbf{s}\rangle(\mathbf{p q} ! j)}{\longrightarrow} T_{j} \\
& \underset{\mathbf{q} \&\langle\mathbf{s}\rangle\left\{l_{i}: T_{i}\right\}_{i \in I} \stackrel{\operatorname{via}\langle\mathbf{s}\rangle(\mathbf{q p} ? j)}{\longrightarrow} T_{j}}{\text { [LR5] }} \\
& \mathbf{p} \hookrightarrow \mathbf{q}:\left\{l_{i}: T_{i}\right\}_{i \in I} \stackrel{\operatorname{via}\langle\mathbf{s}\rangle(\mathbf{p q} ! j)}{\longrightarrow} \mathbf{p} \leftrightarrow \mathbf{q} . j:\left\{l_{i}: T_{i}\right\}_{i \in I} \\
& \mathbf{p} \leftrightarrow \mathbf{q} . j:\left\{l_{i}: T_{i}\right\}_{i \in I} \stackrel{\operatorname{via}\langle\mathbf{s}\rangle(\mathbf{p q} ? j)}{\longrightarrow} T_{j} \\
& \frac{\forall i \in I . T_{i} \stackrel{l}{\longrightarrow} T_{i}^{\prime} \quad \operatorname{subj}(l) \notin\{\mathbf{p}, \mathbf{q}\}}{\mathbf{p} \hookrightarrow \mathbf{q}:\left\{l_{i}: T_{i}\right\}_{i \in I} \stackrel{l}{\longrightarrow} \mathbf{p} \hookrightarrow \mathbf{q}:\left\{l_{i}: T_{i}^{\prime}\right\}_{i \in I}} \text { [LR8] } \\
& \frac{T_{j} \stackrel{l}{\longrightarrow} T_{j}^{\prime} \quad \operatorname{subj}(l) \neq \mathbf{q} \quad \forall i \in I \backslash\{j\} . T_{i}^{\prime}=T_{i}}{\mathbf{p} \leftrightarrow \mathbf{q .} j:\left\{l_{i}: T_{i}\right\}_{i \in I} \stackrel{l}{\longrightarrow} \mathbf{p} \leftrightarrow \mathbf{q} . j:\left\{l_{i}: T_{i}^{\prime}\right\}_{i \in I}} \text { [LR9] } \\
& \frac{l=\mathrm{via}\langle\mathbf{s}\rangle(\cdot) \quad \operatorname{subj}(l) \neq \mathbf{q} \quad \forall i \in I . T_{i} \stackrel{l}{\longrightarrow} T_{i}^{\prime}}{\mathbf{q} \oplus\left\{l_{i}: T_{i}\right\}_{i \in I} \stackrel{l}{\longrightarrow} \mathbf{q} \oplus\left\{l_{i}: T_{i}^{\prime}\right\}_{i \in I}} \text { [LR10] } \\
& \frac{l=\operatorname{via}\langle\mathbf{s}\rangle(\cdot) \quad \operatorname{subj}(l) \neq \mathbf{q} \quad \forall i \in I . T_{i} \stackrel{l}{\longrightarrow} T_{i}^{\prime}}{\mathbf{q} \&\left\{l_{i}: T_{i}\right\}_{i \in I} \longrightarrow \mathbf{l} \text { q \& }\left\{l_{i}: T_{i}^{\prime}\right\}_{i \in I}}
\end{aligned}
$$

Figure 8. LTS over Local Types in RouST

the message $l_{j}$ has been received from $\mathrm{p}$ but not yet routed to $\mathrm{q}$. We extend the projection operator to support this new construct.

$$
\underset{\mathbf{s}}{\mathbf{p}} \mathbf{q} \cdot j:\left\{l_{i}: G_{i}\right\}_{i \in I} \uparrow \mathbf{r}= \begin{cases}\mathbf{p} \&\langle\mathbf{s}\rangle\left\{l_{i}: G_{i} \uparrow \mathbf{r}\right\}_{i \in I} & \text { if } \mathbf{r}=\mathbf{q} \\ \mathbf{p} \leftrightarrow \mathbf{q} \cdot j:\left\{l_{i}: G_{i}\lceil\mathbf{r}\}_{i \in I}\right. & \text { if } \mathbf{r}=\mathbf{p} \\ G_{j} \uparrow \mathbf{r} & \text { otherwise }\end{cases}
$$

LTS Semantics over Local Types. We define the LTS semantics over local types, denoted by $T \stackrel{l}{\longrightarrow} T^{\prime}$, in Fig. 8. We highlight and explain the new rules.

We walk through rules [LR4] and [LR5] from the perspective of role $\mathrm{p}$.

- [LR4] and [LR5] are analogue to [LR1] and [LR2] for sending and receiving messages respectively. The exception is that the new rules match on the router role s on the local type and the routed label.

We walk through rules [LR6], [LR7], [LR10] and [LR11] from the perspective of role $s$.

- [LR6] and [LR7] are analogue to [GR1] and [GR2]. Intuitively, the router $\mathrm{s}$ holds a "global" perspective on the interaction between $\mathrm{p}$ 
and q, which explains the correspondence with the LTS semantics over global types.

- [LR10] and [LR11] allow the router to perform routing actions before handling their own direct communication. The syntax $l=$ $\operatorname{via}\langle\mathbf{s}\rangle(\cdot)$ means that the label $l$ is "of the form" of a routing action, i.e. there exists some $\mathbf{p}, \mathbf{q}, j$ such that $l=\operatorname{via}\langle\mathbf{s}\rangle(\mathbf{p q} ! j)$ or $l=$ $v i a\langle s\rangle(\mathbf{p q} ? j)$. The constraint of $\operatorname{subj}(l) \neq \mathbf{q}$ prevents the violation of the syntactic order of messages sent and received by $q$.

Curious readers can consider the examples $G_{1} \uparrow \mathbf{s}$ and $G_{2} \uparrow \mathbf{s}$ below to see why this constraint is needed.

$$
\begin{aligned}
G_{1} & =\mathbf{s} \rightarrow \mathbf{r}: M 1 . \mathbf{r}-\mathbf{s} \rightarrow \mathbf{q}: M 2 . \text { end } \\
G_{1} \uparrow \mathbf{s} & =\mathbf{r} \oplus M 1 . \mathbf{r} \hookrightarrow \mathbf{q}: M 2 . \text { end } \\
G_{2} & =\mathbf{s} \rightarrow \mathbf{r}: M 1 \cdot \mathbf{p}-\mathbf{s} \rightarrow \mathbf{r}: M 2 . \text { end } \\
G_{2}\lceil\mathbf{s} & =\mathbf{r} \oplus M 1 . \mathbf{p} \hookrightarrow \mathbf{r}: M 2 . \text { end }
\end{aligned}
$$

As for the remaining rules, [LR8] and [LR9] are the local counterparts to [GR4] and [GR5] because the router holds a "global" perspective on the communication, so transitions that do not violate the syntactic order of messages between roles $p$ and $q$ are allowed.

LTS Semantics over Configurations. Let $\mathcal{P}$ denote the set of participants in the communication automaton. Also let $T_{\mathrm{p}}$ denote the local type of a participant $p \in \mathcal{P}$.

A configuration describes the state of the communication automaton with respect to each participant $p \in \mathcal{P}$. By definition of our LTS semantics, this includes intermediate states, so a configuration would also need to express the state of messages in transit.

We inherit the definition from [11], restated in Definition B.3.

Definition B.3 (Configuration). A configuration $s=(\vec{T} ; \vec{w})$ of a system of local types $\left\{T_{\mathrm{p}}\right\}_{\mathrm{p} \in \mathcal{P}}$ is defined as a pair of:

- $\vec{T}=\left(T_{\mathrm{p}}\right)_{\mathrm{p} \in \mathcal{P}}$ is the collection of local types. $T_{\mathrm{p}}$ describes the communication structure from the local perspective of participant $\mathrm{p} \in \mathcal{P}$.

- $\vec{w}=\left(w_{\mathbf{p q}}\right)_{\mathbf{p} \neq \mathbf{q} \in \mathcal{P}}$ is the collection of unbounded buffers. The unbounded buffer $w_{\text {pq }}$ represents a (FIFO) queue of messages sent by $\mathbf{p}$ but not yet received by $\mathbf{q}$.

Remark: The subtyping relation defined on local types (see [11]) can be extended to configurations:

$$
\frac{\vec{w}=\vec{w}^{\prime} \quad \forall \mathbf{p} \in \mathcal{P} \cdot T_{\mathbf{p}}<T_{\mathbf{p}}^{\prime}}{(\vec{T} ; \vec{w}) \prec\left(\vec{T}^{\prime} ; \vec{w}^{\prime}\right)}
$$

We proceed to define the LTS over configurations in Definition B.4, highlighting the extensions required for RouST.

Definition B.4 (LTS Semantics over Configurations). The LTS semantics over configurations is defined by the relation $s_{T} \stackrel{l}{\longrightarrow} s_{T}^{\prime}$. Let $s_{T}=(\vec{T} ; \vec{w})$ and $s_{T}^{\prime}=\left(\vec{T}^{\prime} ; \vec{w}^{\prime}\right)$. We define the specific transitions on $\vec{T}$ and $\vec{w}$ by case analysis on the label $l$.

- $l=\mathbf{p q} ! j$

Then $T_{\mathbf{p}} \stackrel{l}{\longrightarrow} T_{\mathbf{p}}^{\prime}$ because $\mathbf{p}$ initiates the action, so $T_{\mathbf{p}}^{\prime},=T_{\mathbf{p}}$, for all $\mathbf{p}^{\prime} \neq \mathrm{p}$.

The message $j$ is in transit from $\mathbf{p}$ to $\mathbf{q}$, so $w_{\mathbf{p q}}^{\prime}=w_{\mathbf{p q}} \cdot j(j$ is appended to the queue of in-transit messages sent from $p$ to $q)$, and unrelated buffers $w_{\mathbf{p}^{\prime} \mathbf{q}}^{\prime}=w_{\mathrm{pq}}$ are untouched for all $\mathbf{p}^{\prime} \mathbf{q}^{\prime} \neq \mathbf{p q}$.

$$
\begin{aligned}
& \left\langle\mathbf{p} \underset{\mathbf{s}}{\leadsto} \mathbf{p}^{\prime} \cdot j:\left\{l_{i}: G_{i}\right\}_{i \in I}\right\rangle_{\left\{w_{\mathrm{qq}}\right\}_{\mathrm{qq}} \in \mathcal{P}}=\left\langle G_{j}\right\rangle_{\left\{w_{\mathrm{qq}}\right\}_{\mathrm{q}} \in \mathcal{P}}\left[w_{\mathrm{pp}} \mapsto w_{\mathrm{pp}} \cdot j\right] \\
& \left\langle\mathbf{p}-\mathbf{s} \rightarrow \mathbf{p}^{\prime}:\left\{l_{i}: G_{i}\right\}_{i \in I}\right\rangle_{\left\{w_{\mathrm{qq}}\right\}_{\mathrm{qq}} \in \mathcal{P}}=\left\langle G_{i}\right\rangle_{\left\{w_{\mathrm{qq}}{ }^{\prime}\right\}_{\mathrm{qq} \mathbf{q}^{\prime} \in \mathcal{P}}} \text { for } i \in I \\
& \text { since } \forall i, j \in I .\left\langle G_{i}\right\rangle_{\left\{w_{\mathrm{qq}}\right\}_{\mathrm{qq}^{\prime} \in \mathcal{P}}}=\left\langle G_{j}\right\rangle_{\left\{w_{\mathrm{qq}}\right\}_{\mathrm{qq}} \in \mathcal{P}}
\end{aligned}
$$

Figure 9. Projection of Buffer Contents from Global Type in RouST

\section{- $l=\mathbf{p q} ? j$}

Then $T_{\mathbf{q}} \stackrel{l}{\longrightarrow} T_{\mathbf{q}}^{\prime}$ because $\mathbf{q}$ initiates the action, so $T_{\mathbf{p}}^{\prime},=T_{\mathbf{p}}$, for all $\mathrm{p}^{\prime} \neq \mathrm{q}$.

The message $j$ is no longer in transit from $\mathbf{p}$ to $\mathbf{q}$ as it is received by q, so $w_{\mathrm{pq}}=j \cdot w_{\mathrm{pq}}^{\prime}(j$ is removed from the front of the queue of in-transit messages sent from $p$ to $q$ ), and unrelated buffers $w_{\mathbf{p}^{\prime} \mathbf{q}}^{\prime}=w_{\mathrm{pq}}$ are untouched for all $\mathbf{p}^{\prime} \mathbf{q}^{\prime} \neq \mathbf{p q}$.

- $l=\mathrm{via}\langle\mathrm{s}\rangle(\mathrm{pq} ! j)$

Then $T_{\mathrm{p}} \stackrel{l}{\longrightarrow} T_{\mathrm{p}}^{\prime}$ because $\mathrm{p}$ initiates the action. Because the send action is routed, we also need $T_{\mathbf{s}} \stackrel{l}{\longrightarrow} T_{\mathbf{s}}^{\prime}$. This means $T_{\mathbf{p}}^{\prime},=T_{\mathbf{p}}$, for all $p^{\prime} \notin\{p, s\}$.

The message $j$ is in transit from $\mathbf{p}$ to $\mathbf{q}$, so $w_{\mathrm{pq}}^{\prime}=w_{\mathrm{pq}} \cdot j$ and unrelated buffers $w_{\mathbf{p}^{\prime} \mathbf{q}}^{\prime}=w_{\mathrm{pq}}$ are untouched for all $\mathbf{p}^{\prime} \mathbf{q}^{\prime} \neq \mathbf{p q}$.

- $l=v i a\langle s\rangle(p q ? j)$

Then $T_{\mathbf{q}} \stackrel{l}{\longrightarrow} T_{\mathbf{q}}^{\prime}$ because $\mathbf{q}$ initiates the action. Because the receive action is routed, we also need $T_{\mathbf{s}} \stackrel{l}{\longrightarrow} T_{\mathbf{s}}^{\prime}$. This means $T_{\mathbf{p}^{\prime}}^{\prime}$, $T_{\text {p }}$ for all $\mathbf{p}^{\prime} \notin\{\mathbf{q}, \mathbf{s}\}$.

The message $j$ is no longer in transit from $\mathbf{p}$ to $\mathbf{q}$ as it is received by $\mathbf{q}$, so $w_{\mathrm{pq}}=j \cdot w_{\mathrm{pq}}^{\prime}$, and unrelated buffers $w_{\mathbf{p}}^{\prime}{ }^{\prime},=w_{\mathrm{pq}}$ are untouched for all $\mathbf{p}^{\prime} \mathbf{q}^{\prime} \neq \mathbf{p q}$.

Routed actions are carried out by the router, so it makes sense for the local type of the router to also make a step. The semantics of the message buffers for routed actions are the same as their non-routed counterparts; the only difference is that these message buffers are "managed" by the router.

Projection for Configurations. When considering the grammar of global types $G$ extended to include intermediate states, we can obtain the projected configuration from a global type $G$ with participants $\mathcal{P}$ :

$$
\langle G\rangle=\left(\left\{G\lceil\mathbf{p}\}_{\mathbf{p} \in \mathcal{P}} ;\langle G\rangle_{\{\epsilon\}_{\mathbf{q} q^{\prime} \in \mathcal{P}}}\right)\right.
$$

The collection of local types is obtained by projecting $G$ onto each participant $p \in \mathcal{P}$. The contents of the buffers is defined as $\langle G\rangle_{\left\{w_{\mathrm{qq}}\right\}_{\mathrm{qq}{ }^{\prime} \in \mathcal{P}} . \epsilon \text { denotes an empty buffer. We inherit the definitions }}$ presented in [11], and introduce additional rules in Fig. 9.

The semantics of the message buffers for routed actions are the same as their non-routed counterparts, so the projected contents of the buffers for routed communication are the same as those under non-routed communication. 


\section{Appendix for Proofs in $§ 4$}

\section{C.1 Auxiliary Lemmas}

Lemma C.1 (Local LTS Preserves Merge). Let $T_{1}, T_{2}$ be local types. Suppose $T_{1} \sqcap T_{2}$ exists.

$$
\forall l, T_{1}^{\prime}, T_{2}^{\prime} \cdot\left(\left(T_{1} \stackrel{l}{\longrightarrow} T_{1}^{\prime}\right) \wedge\left(T_{2} \stackrel{l}{\longrightarrow} T_{2}^{\prime}\right) \Longrightarrow\left(T_{1}^{\prime} \sqcap T_{2}^{\prime}\right) \text { exists }\right)
$$

Proof. By simultaneous induction on $T_{1} \sqcap T_{2}, T_{1} \stackrel{l}{\longrightarrow} T_{1}^{\prime}$, and $T_{2} \stackrel{l}{\longrightarrow} T_{2}^{\prime}$.

Lemma C.2 (Projection and Participation).

$$
\forall G \text {, p. }(G \uparrow \mathbf{p}=\text { end } \Longleftrightarrow \mathbf{p} \notin \mathrm{pt}(G))
$$

Proof. Prove $(\Longrightarrow)$ by induction on the structure of $G$. Prove $(\Longleftarrow)$ using the contrapositive (stated below) by induction on the derivation of pt $(G)$ :

$$
\mathbf{p} \in \operatorname{pt}(G) \Longrightarrow G \uparrow \mathbf{p} \neq \text { end. }
$$

Lemma C.3 (Encoding Defines Centroid). Given an encoding of global type $G$ with respect to the router role s, the router role is the centroid of the encoded communication:

$$
\llbracket G, s \rrbracket \circledast \mathbf{s} .
$$

Proof. By induction on the structure of $G$.

Lemma C.4 (Encoding and Substitution Permute). Let $G, G^{\prime}$ be global types, and s be a role.

$$
\llbracket G\left[G^{\prime} / \mathbf{t}\right], \mathbf{s} \rrbracket=\llbracket G, \mathbf{s} \rrbracket\left[\llbracket G^{\prime}, \mathbf{s} \rrbracket / t\right]
$$

Proof. By induction on the structure of $G$.

Lemma C.5 (Encoding Preserves Participants).

$$
\forall G, s .(\operatorname{pt}(G) \subseteq \operatorname{pt}(\llbracket G, s \rrbracket))
$$

Proof. The following is logically equivalent:

$$
\forall \mathrm{r}, \mathbf{s .}(\mathbf{r} \in \mathrm{pt}(G) \Longrightarrow \mathrm{r} \in \mathrm{pt}(\llbracket G, s \rrbracket))
$$

We prove this by induction on the structure of $G$.

Lemma C.6 (Encoding Preserves Privacy). The encoding on global types will not introduce non-server roles that were not participants of the original communication.

$$
\forall \mathrm{r}, \mathrm{s}, G . \quad(\mathrm{r} \neq \mathrm{s} \wedge \mathrm{r} \notin \mathrm{pt}(G) \Longrightarrow \mathrm{r} \notin \mathrm{pt}(\llbracket G, \mathrm{~s} \rrbracket))
$$

Proof. The following is logically equivalent.

$$
\forall \mathrm{r}, \mathrm{s}, G . \quad(\mathrm{r} \neq \mathrm{s} \wedge \mathrm{r} \in \mathrm{pt}(\llbracket G, \mathrm{~s} \rrbracket) \Longrightarrow \mathrm{r} \in \mathrm{pt}(G))
$$

We prove this by induction on the structure of $G$, assuming that $\mathrm{r} \neq \mathrm{s}$ for arbitrary roles $\mathrm{r}, \mathrm{s}$.

\section{Proof of Lemma 4.10.}

The projection of an encoded global type $\llbracket G, \mathbf{s} \rrbracket \uparrow \mathbf{r}$ is equal to the encoded local type after projection $\llbracket G \uparrow \mathbf{r}, \mathbf{r}, \mathbf{s} \rrbracket$, with respect to router s, i.e.

$$
\forall \mathrm{r}, \mathrm{s}, G .(\mathrm{r} \neq \mathrm{s} \Longrightarrow \llbracket G, \mathrm{~s} \rrbracket \uparrow \mathrm{r}=\llbracket G \uparrow \mathrm{r}, \mathrm{r}, \mathrm{s} \rrbracket)
$$

Proof. By induction on the structure of $G$, Lemmas C.5 and C.6.

Lemma C.7 (Local Type Encoding Preserves Equality of Projection). $\forall G_{1}, G_{2}, \mathbf{r}, \mathbf{s}$.

$$
\left(\left(G_{1}\lceil\mathbf{r})=\left(G_{2}\lceil\mathbf{r}) \wedge \mathbf{r} \neq \mathbf{s} \Longrightarrow \llbracket G_{1}, \mathbf{s} \rrbracket\left\lceil\mathbf{r}=\llbracket G_{2}, \mathbf{s} \rrbracket\lceil\mathbf{r})\right.\right.\right.\right.
$$

Proof. By consequence from Lemma 4.10.

Take $G_{1}, G_{2}, \mathbf{r}$, s arbitrarily. Assume $\left(G_{1}\lceil\mathbf{r})=\left(G_{2}\lceil\mathbf{r})\right.\right.$ and $\mathbf{r} \neq \mathbf{s}$. We need to show $\llbracket G_{1}, s \rrbracket \uparrow \mathbf{r}=\llbracket G_{2}, s \rrbracket\lceil\mathbf{r}$, but by Lemma 4.10, it is sufficient to show

$$
\llbracket G_{1} \uparrow \mathbf{r}, \mathbf{r}, \mathbf{s} \rrbracket=\llbracket G_{2}\lceil\mathbf{r}, \mathbf{r}, \mathbf{s} \rrbracket .
$$

The result follows by congruence from the assumption.

Lemma C.8 (Encoding on Global Types Preserves Merge). Take global types $G_{1}, G_{2}$ and roles $\mathrm{r}, \mathbf{s}$ such that $\mathbf{r} \neq \mathbf{s}$. Suppose $G_{1}\lceil\mathbf{r}$ and $G_{2}\lceil$ r exist.

$\left(G_{1}\lceil\mathbf{r}) \sqcap\left(G_{2}\lceil\mathbf{r})\right.\right.$ exists $\Longrightarrow\left(\llbracket G_{1}, \mathbf{s} \rrbracket\lceil\mathbf{r}) \sqcap\left(\llbracket G_{2}, \mathbf{s} \rrbracket\lceil\mathbf{r})\right.\right.$ exists

Proof. By induction on the structure of $T_{1} \sqcap T_{2}$, Lemmas C.2 and C.7.

Lemma C.9 (Encoding Preserves Projection). Let $G$ be a global type. $\forall \mathrm{r}, \mathrm{s}$.

$$
G \uparrow \mathrm{r} \text { exists } \Longrightarrow \llbracket G, \mathrm{~s} \rrbracket\lceil\mathrm{r} \text { exists }
$$

Proof. By induction on the structure of $G$.

1. $G=$ end, $G=\mathrm{t}$

As $\llbracket G, \mathrm{~s} \rrbracket=G$, if $G \uparrow \mathbf{r}$ exists, so does $\llbracket G, \mathrm{~s} \rrbracket\lceil\mathrm{r}$.

2. $G=\mu \mathrm{t} . G^{\prime}$

By assumption, $\mu \mathrm{t} . G^{\prime} \uparrow \mathrm{r}$ exists. Note that $G^{\prime} \uparrow \mathrm{r}$ exists regardless of r's participation in $G^{\prime}$.

By induction, $\llbracket G^{\prime}, \mathbf{s} \rrbracket \uparrow \mathbf{r}$ exists.

To show $\llbracket \mu \mathrm{t} . G^{\prime}, \mathrm{s} \rrbracket \uparrow \mathrm{r}$ exists, consider $\mathbf{r}$ by case:

- $r \in \operatorname{pt}\left(\llbracket G^{\prime}, s \rrbracket\right)$ :

$$
\llbracket \mu \mathrm{t} . G^{\prime}, \mathrm{s} \rrbracket\left\lceil\mathbf{r}=\mu \mathrm{t} . \llbracket G^{\prime}, \mathbf{s} \rrbracket\left\lceil\mathbf{r}=\mu \mathrm{t} .\left(\llbracket G^{\prime}, \mathbf{s} \rrbracket\lceil\mathbf{r})\right.\right.\right.
$$

As $\llbracket G^{\prime}, s \rrbracket \uparrow r$ exists, so does $\llbracket \mu \mathrm{t} . G^{\prime}, s \rrbracket \uparrow \mathrm{r}$.

- $\mathrm{r} \notin \mathrm{pt}\left(\llbracket G^{\prime}, \mathrm{s} \rrbracket\right)$ :

$$
\llbracket \mu \mathrm{t} . G^{\prime}, \mathrm{s} \rrbracket\left\lceil\mathrm{r}=\mu \mathrm{t} . \llbracket G^{\prime}, \mathrm{s} \rrbracket \uparrow \mathrm{r}=\mathrm{end}\right.
$$

3. $G=\mathbf{p} \rightarrow \mathbf{q}:\left\{l_{i}: G_{i}\right\}_{i \in I}$

To determine $\llbracket G, \mathbf{s} \rrbracket$, consider s by case:

- $s \in\{\mathbf{p}, \mathbf{q}\}$ :

Then $\llbracket G, s \rrbracket=\mathbf{p} \rightarrow \mathbf{q}:\left\{l_{i}: \llbracket G_{i}, s \rrbracket\right\}_{i \in I}$.

To show $\llbracket G, s \rrbracket\lceil\mathbf{r}$ exists, consider $\mathbf{r}$ by case:

- $\mathbf{r}=\mathbf{p}$ : Then $G \uparrow \mathbf{p}=\mathbf{q} \oplus\left\{l_{i}: G_{i} \uparrow \mathbf{p}\right\}_{i \in I}$.

By induction, $\llbracket G_{i}, \mathbf{s} \rrbracket \uparrow \mathbf{p}$ exists for $i \in I$.

$\llbracket G, \mathbf{s} \rrbracket \uparrow \mathbf{p}=\mathbf{q} \oplus\left\{l_{i}: \llbracket G_{i}, \mathbf{s} \rrbracket \uparrow \mathbf{p}\right\}_{i \in I}$.

As projections of the encoded continuations exist, so does $\llbracket G, \mathbf{s} \rrbracket \mathbf{p}$.

$-r=q$ : Follows similarly from above. 
- $\mathbf{r} \notin\{\mathbf{p}, \mathbf{q}\}$ : Then $G \uparrow \mathbf{r}=\prod_{i \in I} G_{i} \uparrow \mathbf{r}$, so the merge exists.

By induction, $\llbracket G_{i}, \mathrm{~s} \rrbracket \uparrow \mathrm{r}$ exists for $i \in I$.

$\llbracket G, \mathbf{s} \rrbracket \uparrow \mathbf{r}=\prod_{i \in I} \llbracket G_{i}, \mathbf{s} \rrbracket \uparrow \mathbf{r}$, and this merge exists by Lemma C.8.

- $\mathbf{s} \notin\{\mathbf{p}, \mathbf{q}\}$ :

Then $\llbracket G, \mathbf{s} \rrbracket=\mathbf{p}-\mathbf{s} \rightarrow \mathbf{q}:\left\{l_{i}: \llbracket G_{i}, \mathbf{s} \rrbracket\right\}_{i \in I}$.

To show $\llbracket G, s \rrbracket \uparrow r$ exists, consider $\mathbf{r}$ by case:

- $\mathbf{r}=\mathbf{p}$ : Then $G \uparrow \mathbf{p}=\mathbf{q} \oplus\left\{l_{i}: G_{i} \uparrow \mathbf{p}\right\}_{i \in I}$.

By induction, $\llbracket G_{i}, \mathbf{s} \rrbracket \uparrow \mathbf{p}$ exists for $i \in I$.

$\llbracket G, \mathbf{s} \rrbracket \uparrow \mathbf{p}=\mathbf{q} \oplus\langle\mathbf{s}\rangle\left\{l_{i}: \llbracket G_{i}, \mathbf{s} \rrbracket \uparrow \mathbf{p}\right\}_{i \in I}$.

As projections of the encoded continuations exist, so does $\llbracket G, s \rrbracket \uparrow \mathbf{p}$.

$-\mathrm{r}=\mathrm{q}$ : Follows similarly from above.

- $\mathbf{r}=\mathbf{s}$ : Then $G \uparrow \mathbf{s}=\prod_{i \in I} G_{i} \uparrow \mathbf{s}$.

By induction, $\llbracket G_{i}, \mathrm{~s} \rrbracket\lceil$ p exists for $i \in I$.

$\llbracket G, \mathbf{s} \rrbracket \mathbf{s}=\mathbf{p} \hookrightarrow \mathbf{q}:\left\{l_{i}: \llbracket G_{i}, \mathbf{s} \rrbracket \uparrow \mathbf{s}\right\}_{i \in I}$.

As projections of the encoded continuations exist, so does $\llbracket G, \mathbf{s} \rrbracket \mathbf{s}$.

- $\mathbf{r} \notin\{\mathbf{p}, \mathbf{q}, \mathbf{s}\}$ : Then $G \uparrow \mathbf{r}=\prod_{i \in I} G_{i} \uparrow \mathbf{r}$, so the merge exists.

By induction, $\llbracket G_{i}, \mathrm{~s} \rrbracket \uparrow \mathrm{r}$ exists for $i \in I$.

$\llbracket G, \mathbf{s} \rrbracket \uparrow \mathrm{r}=\prod_{i \in I} \llbracket G_{i}, \mathbf{s} \rrbracket \uparrow \mathrm{r}$, and this merge exists by Lemma C.8.

\section{C.2 Proof of Theorem 4.6}

Let $G$ be a global type with participants $\mathcal{P}=$ pt $(G)$, and let $\vec{T}=\left\{G\lceil\mathbf{p}\}_{\mathbf{p} \in \mathcal{P}}\right.$ be the local types projected from $G$. Then $G \approx(\vec{T}, \vec{\epsilon})$.

Proof. Direct consequence of Lemma C.10.

Lemma C.10 (Step Equivalence). For all global types $G$ and configurations $s$, if $\langle G\rangle<s$, then $G \stackrel{l}{\longrightarrow} G^{\prime} \Longleftrightarrow s \stackrel{l}{\longrightarrow} s^{\prime}$ such that $\left\langle G^{\prime}\right\rangle \prec s^{\prime}$.

Proof. By induction on the possible transitions in the LTSs over global types (to prove $\Longrightarrow$, i.e. soundness) and configurations (to prove $\Longleftarrow$, i.e. completeness).

Notation conventions. We use the following notation for decomposing configurations and projected configurations.

$$
\begin{aligned}
s & =\left\{T_{\mathrm{q}}\right\}_{\mathrm{q} \in \mathcal{P}},\left\{w_{\mathrm{qq}^{\prime}}\right\}_{\mathrm{qq}^{\prime} \in \mathcal{P}} \\
s^{\prime} & =\left\{T_{\mathbf{q}}^{\prime}\right\}_{\mathrm{q} \in \mathcal{P}},\left\{w_{\mathbf{q q}^{\prime}}^{\prime}\right\}_{\mathrm{qq}^{\prime} \in \mathcal{P}} \\
\langle G\rangle & =\left\{\hat{T_{\mathrm{q}}}\right\}_{\mathrm{q} \in \mathcal{P}},\left\{\hat{w}_{\mathbf{q q}^{\prime}}\right\}_{\mathrm{qq}^{\prime} \in \mathcal{P}} \\
\left\langle G^{\prime}\right\rangle & =\left\{\hat{T}_{\mathbf{q}}^{\prime}\right\}_{\mathbf{q} \in \mathcal{P}},\left\{\hat{w}^{\prime}{ }_{\mathrm{qq}}\right\}_{\mathrm{qq}^{\prime} \in \mathcal{P}}
\end{aligned}
$$

\section{Soundness}

By rule induction on LTS semantics over global types.

For each transition $G \stackrel{l}{\longrightarrow} G^{\prime}$, we take the configuration $s=\langle G\rangle$, derive $G \stackrel{l}{\longrightarrow} G^{\prime}$ and $s \stackrel{l}{\longrightarrow} s^{\prime}$ under the respective LTSs, and show that $s^{\prime} \prec\left\langle G^{\prime}\right\rangle$.

The proofs for rules [GR1-5] are the same as in [11]. We focus on the new rules introduced for routing.

- [GR6], where $G=\mathbf{p}-\mathbf{s} \rightarrow \mathbf{p}^{\prime}:\left\{l_{i}: G_{i}\right\}_{i \in I}$,

$G^{\prime}=\mathbf{p} \underset{\mathbf{s}}{\mathbf{p}^{\prime}} \cdot j:\left\{l_{i}: G_{i}\right\}_{i \in I}$ with $l=\mathrm{via}\langle\mathbf{s}\rangle\left(\mathbf{p} \mathbf{p}^{\prime} ! j\right)$.
Then $s=\langle G\rangle$ where

$$
\begin{aligned}
T_{\mathbf{p}} & =\mathbf{p}^{\prime} \oplus\langle\mathbf{s}\rangle\left\{l_{i}: G_{i}\lceil\mathbf{p}\}_{i \in I}\right. \\
T_{\mathbf{p}}, & =\mathbf{p} \&\langle\mathbf{s}\rangle\left\{l_{i}: G_{i}\left\lceil\mathbf{p}^{\prime}\right\}_{i \in I}\right. \\
T_{\mathbf{s}} & =\mathbf{p} \hookrightarrow \mathbf{p}^{\prime}:\left\{l_{i}: G_{i}\lceil\mathbf{s}\}_{i \in I}\right. \\
T_{\mathbf{r}} & =\prod_{i \in I} G_{i}\left\lceil\mathbf{r} \text { for } \mathbf{r} \notin\left\{\mathbf{p}, \mathbf{p}^{\prime}, \mathbf{s}\right\}\right. \\
\left\{w_{\mathbf{q q}}\right\}_{\mathbf{q q}} \in \mathcal{P} & =\left\langle G_{i}\right\rangle_{\{\vec{\epsilon}\}} \text { for some } i \in I
\end{aligned}
$$

Global transition: We have

$$
\begin{aligned}
\hat{T_{\mathbf{p}}^{\prime}} & =\mathbf{p} \&\langle\mathbf{s}\rangle\left\{l_{i}: G_{i}\left\lceil\mathbf{p}^{\prime}\right\}_{i \in I}\right. \\
\hat{T_{\mathbf{s}}^{\prime}} & =\mathbf{p} \leftrightarrow \mathbf{p}^{\prime} \cdot j:\left\{l_{i}: G_{i} \uparrow \mathbf{s}\right\}_{i \in I} \\
\hat{T_{\mathbf{r}}^{\prime}} & =G_{j} \uparrow \mathbf{r} \text { for } \mathbf{r} \notin\left\{\mathbf{p}^{\prime}, \mathbf{s}\right\} \\
\left\{\hat{w}^{\prime}{ }_{\mathrm{qq}}\right\}_{\mathbf{q q}} \in \mathcal{P} & =\left\langle G_{i}\right\rangle_{\{\vec{\epsilon}\}\left[w_{\mathrm{pp}} \mapsto w_{\mathrm{pp}} \cdot j\right]} \text { for some } i \in I
\end{aligned}
$$

So, $\hat{w}_{\mathbf{q q}}^{\prime}=w_{\mathbf{q q}}$, for $\mathbf{q q}^{\prime} \neq \mathbf{p p}$ ' and $\hat{w}_{\mathbf{p p}}^{\prime}=w_{\mathrm{pp}} \cdot j$.

Configuration transition: Take $T_{\mathrm{r}}^{\prime}=T_{\mathrm{r}}$ for $\mathbf{r} \notin\{\mathbf{p}, \mathbf{s}\}$.

By [LR4], $T_{\mathbf{p}} \stackrel{l}{\longrightarrow} T_{\mathbf{p}}^{\prime}$ where $T_{\mathbf{p}}^{\prime}=G_{j} \uparrow \mathbf{p}$.

By [LR6], $T_{\mathbf{s}} \stackrel{l}{\longrightarrow} T_{\mathbf{s}}^{\prime}$ where $T_{\mathbf{s}}^{\prime}=\mathbf{p} \leftrightarrow \mathbf{p}^{\prime} . j:\left\{l_{i}: G_{i} \uparrow \mathbf{s}\right\}_{i \in I}$.

Also, $w^{\prime}{ }_{\mathrm{qq}}{ }^{\prime}=w_{\mathrm{qq}}$, for $\mathbf{q q}^{\prime} \neq \mathbf{p p}$ ' and $w_{\mathrm{pp}}^{\prime}=w_{\mathrm{pp}} \cdot j$.

Correspondence: We have $w^{\prime}{ }_{\mathrm{qq}}{ }^{\prime}=\hat{w}_{\mathrm{qq}}$, for $\mathbf{q q} \mathrm{q}^{\prime} \in \mathcal{P}$ and $T_{\mathrm{q}}^{\prime}=$ $\hat{T_{\mathbf{q}}}$ for $q \in\left\{p, p^{\prime}, s\right\}$.

For $\mathbf{q} \notin\left\{\mathbf{p}, \mathbf{p}^{\prime}, \mathbf{s}\right\}$, we have

$$
T_{\mathbf{q}}^{\prime}=\prod_{i \in I} G_{i} \uparrow \mathbf{q}<G_{j} \uparrow \mathbf{q}=\hat{T}_{\mathbf{q}}
$$

So $s^{\prime} \prec\left\langle G^{\prime}\right\rangle$.

- [GR7] where $G=\mathbf{p} \underset{\text { s }}{m} \mathbf{p}^{\prime} . j:\left\{l_{i}: G_{i}\right\}_{i \in I}, G^{\prime}=G_{j}$, and $l=\mathrm{via}\langle\mathbf{s}\rangle(\mathbf{p p}$ '? $j$ ) Then $s=\langle G\rangle$ where

$$
\begin{aligned}
T_{\mathbf{p}}, & =\mathbf{p} \&\langle\mathbf{s}\rangle\left\{l_{i}: G_{i}\left\lceil\mathbf{p}^{\prime}\right\}_{i \in I}\right. \\
T_{\mathbf{s}} & =\mathbf{p} \leftrightarrow \mathbf{p}^{\prime} \cdot j:\left\{l_{i}: G_{i} \uparrow \mathbf{s}\right\}_{i \in I} \\
T_{\mathbf{r}} & =G_{j} \uparrow \mathbf{r} \text { for } \mathbf{r} \notin\left\{\mathbf{p}^{\prime}, \mathbf{s}\right\} \\
\left\{w_{\mathbf{q q}}\right\}_{\mathbf{q q}} \in \mathcal{P} & =\left\langle G_{j}\right\rangle_{\{\vec{\epsilon}\}\left[w_{\mathbf{p} \mathbf{p}^{\prime}} \mapsto w_{\mathbf{p p}}, j\right]}
\end{aligned}
$$

Global transition: We have

$$
\begin{aligned}
\hat{T}_{\mathrm{r}}^{\prime} & =G_{j} \uparrow \mathbf{r} \text { for } \mathbf{r} \in \mathcal{P} \\
\left\{{\hat{w^{\prime}}}_{\mathrm{qq}^{\prime}}\right\}_{\mathrm{qq}^{\prime} \in \mathcal{P}} & =\left\langle G_{j}\right\rangle_{\{\vec{\epsilon}\}}
\end{aligned}
$$

So, ${\hat{w^{\prime}}}_{\mathbf{q q}}{ }^{\prime}=w_{\mathrm{qq}}$, for $\mathbf{q q}^{\prime} \neq \mathbf{p} \mathbf{p}^{\prime}$ and $w_{\mathrm{pp}}=j \cdot \hat{w}_{\mathrm{pp}}$ '

Configuration transition: Take $T_{\mathrm{r}}^{\prime}=T_{\mathrm{r}}$ for $\mathbf{r} \notin\left\{\mathbf{p}^{\prime}, \mathbf{s}\right\}$.

By [LR5], $T_{\mathbf{p}} \stackrel{l}{\longrightarrow} T_{\mathbf{p}}^{\prime}$ where $T_{\mathbf{p}}^{\prime}=G_{j} \uparrow \mathbf{p}$.

By [LR7], $T_{\mathbf{s}} \stackrel{l}{\longrightarrow} T_{\mathbf{s}}^{\prime}$ where $T_{\mathbf{s}}^{\prime}=G_{j} \uparrow \mathbf{s}$.

Also, $w^{\prime}{ }_{\mathrm{qq}}{ }^{\prime}=w_{\mathrm{qq}}$, for $\mathbf{q q} \mathbf{q}^{\prime} \neq \mathbf{p} \mathbf{p}^{\prime}$ and $w_{\mathrm{pp}}=j \cdot w_{\mathrm{pp}}^{\prime}$ '

Correspondence: We have $w^{\prime}{ }_{\mathrm{qq}}{ }^{\prime}=\hat{w}_{\mathrm{qq}}$ ' for $\mathbf{q q} \mathrm{q}^{\prime} \in \mathcal{P}$ and $T_{\mathrm{q}}^{\prime}=$

$\hat{T_{\mathrm{q}}}$ for $\mathrm{q} \in \mathcal{P}$.

So, $s^{\prime}=\left\langle G^{\prime}\right\rangle$.

- [GR8] where $G=\mathbf{p}-\mathbf{s} \rightarrow \mathbf{p}^{\prime}:\left\{l_{i}: G_{i}\right\}_{i \in I}$,

$G^{\prime}=\mathbf{p}-\mathbf{s} \rightarrow \mathbf{p}^{\prime}:\left\{l_{i}: G_{i}^{\prime}\right\}_{i \in I}$

By hypothesis, $\forall i \in I$. $G_{i} \stackrel{l}{\longrightarrow} G_{i}^{\prime}$ and $\operatorname{subj}(l) \notin\left\{\mathbf{p}, \mathbf{p}^{\prime}\right\}$.

By induction, $\forall i \in I .\left\langle G_{i}\right\rangle \stackrel{l}{\longrightarrow}\left\langle G_{i}^{\prime}\right\rangle$. 
To show that $\langle G\rangle \stackrel{l}{\longrightarrow}\left\langle G^{\prime}\right\rangle$, it is sufficient to show that $G \uparrow \mathbf{q} \stackrel{l}{\longrightarrow} G^{\prime} \uparrow \mathbf{q}$ for $\mathbf{q}=\operatorname{subj}(l)$, since the projections for $\mathbf{q}^{\prime} \neq \operatorname{subj}(l)$ remain the same.

We know $G \uparrow \mathbf{q}=\prod_{i \in I} G_{i} \uparrow \mathbf{q}$ and $G^{\prime} \uparrow \mathbf{q}=\prod_{i \in I} G_{i}^{\prime} \uparrow \mathbf{q}$.

By induction, $\prod_{i \in I} G_{i} \uparrow \mathbf{q} \stackrel{l}{\longrightarrow} \prod_{i \in I} G_{i}^{\prime} \uparrow \mathbf{q}$, so $G \uparrow \mathbf{q} \stackrel{l}{\longrightarrow} G^{\prime} \uparrow \mathbf{q}$.

- [GR9] where $G=\mathbf{p} \leadsto \mathbf{s}$ $G^{\prime}=\mathbf{p} \underset{\mathbf{s}}{\rightsquigarrow} \mathbf{p}^{\prime} \cdot j:\left\{l_{i}: G_{i}^{\prime}\right\}_{i \in I^{\prime}}$.

By hypothesis, $G_{j} \stackrel{l}{\longrightarrow} G_{j}^{\prime}, \mathbf{p}^{\prime} \neq \operatorname{subj}(l)$, and $\forall i \in I \backslash\{j\} . G_{i}^{\prime}=G_{i}$. By induction, $\left\langle G_{j}\right\rangle \stackrel{l}{\longrightarrow}\left\langle G_{j}^{\prime}\right\rangle$.

To show that $\langle G\rangle \stackrel{l}{\longrightarrow}\left\langle G^{\prime}\right\rangle$, it is sufficient to show that $G \uparrow \mathbf{q} \stackrel{l}{\longrightarrow} G^{\prime} \uparrow \mathbf{q}$ for $\mathbf{q}=\operatorname{subj}(l)$, since the projections for $\mathbf{q}^{\prime} \neq \operatorname{subj}(l)$ remain the same.

We know $G \uparrow \mathbf{q}=G_{j} \uparrow \mathbf{q}$ and $G^{\prime} \uparrow \mathbf{q}=G_{j}^{\prime} \uparrow \mathbf{q}$.

By induction, $G_{j} \uparrow \mathbf{q} \stackrel{l}{\longrightarrow} G_{j}^{\prime} \uparrow \mathbf{q}$, so $G \uparrow \mathbf{q} \stackrel{l}{\longrightarrow} G^{\prime} \uparrow \mathbf{q}$.

\section{Completeness}

By considering the possible transitions in the LTS over configurations, defined by case analysis on the possible labels $l$.

For each transition $s \stackrel{l}{\longrightarrow} s^{\prime}$, we take the configuration $s$ from the reduction rule, infer the structure of the global type $G$ such that $s=\langle G\rangle$, derive $s \stackrel{l}{\longrightarrow} s^{\prime}$ and $G \stackrel{l}{\longrightarrow} G^{\prime}$ under the respective LTSs, and show that $s^{\prime} \prec\left\langle G^{\prime}\right\rangle$.

The proofs for $l=\mathbf{p q} ! j$ and $l=\mathbf{p q} ? j$ are the same as in $\S 4.2$ of [11]. We focus on the new labels introduced for routing.

- $l=\operatorname{via}\langle\mathrm{s}\rangle(\mathrm{pq} ! j)$ :

Then $T_{\mathbf{p}}=\mathbf{q} \oplus\langle\mathbf{s}\rangle\left\{l_{i}: G_{i} \uparrow \mathbf{p}\right\}_{i \in I}$.

Also, $T_{\mathrm{s}}$ contains $\mathbf{p} \hookrightarrow \mathbf{q}:\left\{l_{i}: G_{i} \uparrow \mathbf{s}\right\}_{i \in I}$ as subterm. We denote this subterm $\tilde{T}_{\mathrm{s}}$.

By definition of projection, $G$ has $\mathbf{p}-\mathbf{s} \rightarrow \mathbf{q}:\left\{l_{i}: G_{i}\right\}_{i \in I}$ as subterm. We denote this subterm $\tilde{G}$.

Also by definition of projection, no action in $G$ will involve p before $\tilde{G}$.

\section{Configuration transition:}

By [LR4], $T_{\mathbf{p}} \stackrel{l}{\longrightarrow} T_{\mathbf{p}}^{\prime}$, where $T_{\mathbf{p}}^{\prime}=G_{j} \uparrow \mathbf{p}$.

By [LR6], $\tilde{T}_{\mathrm{s}} \stackrel{l}{\longrightarrow} \tilde{T}_{\mathrm{s}}^{\prime}$, where $\tilde{T}_{\mathrm{s}}^{\prime}=\mathrm{p} \leftrightarrow \mathrm{q} \cdot j:\left\{l_{i}: G_{i} \uparrow \mathbf{s}\right\}_{i \in I}$.

We get $T_{\mathrm{s}} \stackrel{l}{\longrightarrow} T_{\mathrm{s}}^{\prime}$ by inversion lemma, as illustrated below.

$$
\frac{\frac{\tilde{T}_{\mathrm{s}} \stackrel{l}{\longrightarrow} \tilde{T}_{\mathrm{s}}^{\prime}}{[\mathrm{LR} 6]}}{T_{\mathrm{s}} \stackrel{l}{\longrightarrow} T_{\mathrm{s}}^{\prime}}[\operatorname{Lr} 8,9,10,11] \text { as needed }
$$

Global transition:

By [GR6], $\tilde{G} \stackrel{l}{\longrightarrow} \tilde{G}^{\prime}$, where $\tilde{G}^{\prime}=\mathbf{p} \underset{\mathbf{s}}{\mathfrak{q} .} j:\left\{l_{i}: G_{i}\right\}_{i \in I}$.

We get $G \stackrel{l}{\longrightarrow} G^{\prime}$ by inversion lemma, as illustrated below.

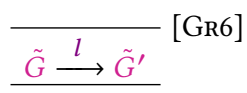

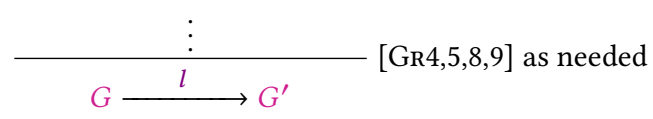

Correspondence: Since the projections for $p^{\prime} \notin\{p, s\}$ are unchanged, it is sufficient to show that $T_{\mathrm{p}}^{\prime}<\left(\tilde{G}^{\prime} \uparrow \mathbf{p}\right)$ and $\tilde{T}_{\mathrm{s}}^{\prime}<\left(\tilde{G}^{\prime} \uparrow \mathrm{s}\right)$.

$$
\begin{aligned}
& \tilde{G}^{\prime} \uparrow \mathbf{p}=G_{j} \uparrow \mathbf{p}=T_{\mathbf{p}}^{\prime} \\
& \tilde{G}^{\prime} \uparrow \mathbf{s}=\mathbf{p} \hookrightarrow \mathbf{q} \cdot j:\left\{l_{i}: G_{i} \uparrow \mathbf{s}\right\}_{i \in I}=\tilde{T}_{\mathbf{s}}^{\prime}
\end{aligned}
$$

- $l=v i a\langle s\rangle(p q ? j)$ :

Then $T_{\mathbf{q}}=\mathbf{p} \&\langle\mathbf{s}\rangle\left\{l_{i}: G_{i}\lceil\mathbf{q}\}_{i \in I}\right.$.

Also, $T_{\mathbf{s}}$ contains $\mathbf{p} \leftrightarrow$ q. $j:\left\{l_{i}: G_{i} \uparrow \mathbf{s}\right\}_{i \in I}$ as subterm. We denote this subterm $\tilde{T}_{\mathrm{s}}$.

By definition of projection, $G$ has $\mathbf{p} \underset{\mathbf{s}}{\sim} \mathbf{q} \cdot j:\left\{l_{i}: G_{i}\right\}_{i \in I}$ as subterm. We denote this subterm $\tilde{G}$.

Also by definition of projection, no action in $G$ will involve $\mathbf{q}$ before $\tilde{G}$.

\section{Configuration transition:}

By [LR5], $T_{\mathbf{q}} \stackrel{l}{\longrightarrow} T_{\mathbf{q}}^{\prime}$, where $T_{\mathbf{q}}^{\prime}=G_{j} \uparrow \mathbf{q}$.

By [LR7], $\tilde{T}_{\mathrm{s}} \stackrel{l}{\longrightarrow} \tilde{T}_{\mathrm{s}}^{\prime}$, where $\tilde{T}_{\mathrm{s}}^{\prime}=G_{j} \uparrow \mathbf{s}$.

We get $T_{\mathrm{s}} \stackrel{l}{\longrightarrow} T_{\mathrm{s}}^{\prime}$ by inversion lemma, as illustrated below.

$$
\frac{\frac{\tilde{T}_{\mathrm{s}} \stackrel{l}{\longrightarrow} \tilde{T}_{\mathrm{s}}^{\prime}}{\text { [LR7] }}}{T_{\mathrm{s}} \stackrel{l}{\longrightarrow} T_{\mathrm{s}}^{\prime}}[\mathrm{LR} 8,9,10,11] \text { as needed }
$$

\section{Global transition:}

By [GR7], $\tilde{G} \stackrel{l}{\longrightarrow} \tilde{G}^{\prime}$, where $\tilde{G}^{\prime}=G_{j}$.

We get $G \stackrel{l}{\longrightarrow} G^{\prime}$ by inversion lemma, as illustrated below.

$$
\begin{aligned}
& \stackrel{\stackrel{l}{\longrightarrow} \tilde{G}^{\prime}}{\text { [GR7] }}
\end{aligned}
$$

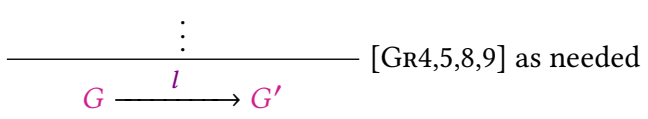

Correspondence: Since the projections for $\mathbf{p}^{\prime} \notin\{\mathrm{q}, \mathrm{s}\}$ are unchanged, it is sufficient to show that $T_{\mathbf{q}}^{\prime}<\left(\tilde{G}^{\prime} \uparrow \mathbf{q}\right)$ and $\tilde{T}_{\mathbf{s}}^{\prime}<\left(\tilde{G}^{\prime} \uparrow \mathbf{s}\right)$.

$$
\begin{aligned}
& \tilde{G}^{\prime} \uparrow \mathbf{q}=G_{j} \uparrow \mathbf{q}=T_{\mathbf{q}}^{\prime} \\
& \tilde{G}^{\prime} \uparrow \mathbf{s}=G_{j} \uparrow \mathbf{s}=\tilde{T}_{\mathbf{s}}^{\prime}
\end{aligned}
$$

\section{C.3 Proof of Theorem 4.7}

Let $G$ be a global type. Suppose $G$ is well-formed with respect to some router s, i.e. wellFormed ${ }^{R}(G, s)$.

$$
\forall G^{\prime} .\left(G \rightarrow^{*} G^{\prime} \Longrightarrow\left(G^{\prime}=\text { end }\right) \vee \exists G^{\prime \prime}, l .\left(G^{\prime} \stackrel{l}{\longrightarrow} G^{\prime \prime}\right)\right)
$$

Proof. Direct consequence of Lemmas C.11 and C.12.

Lemma C.11 (Preservation of Well-formedness). Let $G$ be a global type. Suppose $G$ is well-formed with respect to some router s, i.e. wellFormed ${ }^{R}(G, s)$.

$$
\forall G^{\prime}, \text { l. }\left(G \stackrel{l}{\longrightarrow} G^{\prime} \Longrightarrow \text { wellFormed }^{R}\left(G^{\prime}, \mathrm{s}\right)\right)
$$

Proof. By rule induction on $G \stackrel{l}{\longrightarrow} G^{\prime}$.

For each transition, we show the two conjuncts for well-formedness, wellFormed ${ }^{R}\left(G^{\prime}, \mathrm{s}\right)$ :

(1) $G^{\prime} \uparrow \mathbf{r}$ exists for $\mathbf{r}$ such that $G \uparrow \mathbf{r}$ exists; and, (2) $G^{\prime} \circledast \mathbf{s}$. 
- [GR1], where $G=\mathbf{p} \rightarrow \mathbf{q}:\left\{l_{i}: G_{i}\right\}_{i \in I}$,

$G^{\prime}=\mathbf{p} \rightsquigarrow \mathbf{q} \cdot j:\left\{l_{i}: G_{i}\right\}_{i \in I}$,

and $l=\mathrm{pq} ! j$.

(1). We know $G \uparrow \mathrm{r}$ by assumption. To show $G^{\prime} \uparrow \mathbf{r}$, consider $\mathbf{r}$ by case:

- $\mathbf{r}=\mathbf{p}$ : Then $G \uparrow \mathbf{p}=\mathbf{q} \oplus\left\{l_{i}: G_{i} \uparrow \mathbf{p}\right\}_{i \in I}$, so $\forall i \in I . G_{i} \uparrow \mathbf{p}$ exists.

$G^{\prime} \uparrow \mathbf{p}=G_{j} \uparrow \mathbf{p}$, which exists as $j \in I$.

- $\mathbf{r}=\mathbf{q}$ : Then $G^{\prime} \uparrow \mathbf{q}=\mathbf{p} \&\left\{l_{i}: G_{i} \uparrow \mathbf{q}\right\}_{i \in I} G \uparrow \mathbf{q}$, which exists.

- $\mathbf{r} \notin\{\mathbf{p}, \mathbf{q}\}$ : Then $G \uparrow \mathbf{r}=\prod_{i \in I} G_{i} \uparrow \mathbf{r}$, so $\forall i \in I$. $G_{i} \uparrow \mathbf{r}$ exists.

$G^{\prime} \uparrow \mathbf{r}=G_{j} \uparrow \mathbf{r}$, which exists as $j \in I$.

(2). We know $G \circledast \mathrm{s}$ by assumption. We deduce $G^{\prime} \circledast$ s by consequence.

$$
\begin{aligned}
G \circledast \mathbf{s} & \Longrightarrow \mathbf{s} \in\{\mathbf{p}, \mathbf{q}\} \wedge \bigwedge_{i \in I} G_{i} \circledast \mathbf{s} \\
& \Longrightarrow \mathbf{s} \in\{\mathbf{p}, \mathbf{q}\} \wedge G_{j} \circledast \mathbf{s} \\
& \Longrightarrow G^{\prime} \circledast \mathbf{s}
\end{aligned}
$$

- [GR2], where $G=\mathbf{p} \rightsquigarrow \mathbf{q} \cdot j:\left\{l_{i}: G_{i}\right\}_{i \in I}, G^{\prime}=G_{j}, l=\mathbf{p q}$ ? $j$.

(1). We know $G \uparrow r$ by assumption. To show $G^{\prime} \uparrow r$, consider $r$ by case:

- $\mathbf{r}=\mathbf{p}$ : Then $G^{\prime} \uparrow \mathbf{p}=G_{j} \uparrow \mathbf{p}=G \uparrow \mathbf{p}$, which exists.

- $\mathbf{r}=\mathbf{q}$ : Then $G \uparrow \mathbf{q}=\mathbf{p} \&\left\{l_{i}: G_{i} \uparrow \mathbf{q}\right\}_{i \in I}$, so $\forall i \in I$. $G_{i} \uparrow \mathbf{q}$ exists.

$G^{\prime} \uparrow \mathbf{q}=G_{j} \uparrow \mathbf{q}$, which exists as $j \in I$.

- $\mathbf{r} \notin\{\mathbf{p}, \mathbf{q}\}$ : Then $G^{\prime} \uparrow \mathbf{r}=G_{j} \uparrow \mathbf{r}=G \uparrow \mathbf{r}$, which exists.

(2). We know $G \circledast \mathrm{s}$ by assumption. We deduce $G^{\prime} \circledast$ s by consequence.

$$
G \circledast \mathbf{s} \Longrightarrow \mathbf{s} \in\{\mathbf{p}, \mathbf{q}\} \wedge G_{j} \circledast \mathbf{s} \Longrightarrow G^{\prime} \circledast \mathbf{s}
$$

- [GR3], where $\mu \mathrm{t}$.G $\stackrel{l}{\longrightarrow} G^{\prime}$. By hypothesis, $G[\mu \mathrm{t} . G / \mathrm{t}] \stackrel{l}{\longrightarrow} G^{\prime}$. We first show that wellFormed ${ }^{R}(G[\mu \mathrm{t} . G / \mathrm{t}], \mathbf{s})$.

(1) $\mu \mathrm{t} . G \uparrow \mathbf{r}$ exists for some $\mathbf{r}$.

Note that $G \uparrow r$ exists regardless of r's participation in $G$.

- If $\mathbf{r} \in \operatorname{pt}(G)$, then $\mu$ t. $G\lceil\mathbf{r}=\mu \mathrm{t} . G\lceil\mathbf{r}$, so $G\lceil\mathbf{r}$ exists.

- Otherwise, $G \uparrow \mathrm{r}=$ end, which exists.

Projection is homomorphic under recursion, so $G[\mu \mathrm{t} . G / \mathrm{t}] \uparrow \mathbf{r}$ exists.

(2) By assumption, $(\mu \mathrm{t} . G) \circledast \mathbf{s}$, so $G \circledast \mathbf{s}$.

The $\circledast$ relation is also homomorphic under recursion, so we get $G[\mu \mathrm{t} . G / \mathbf{t}] \circledast \mathbf{s}$.

We conclude by induction to obtain wellFormed ${ }^{R}\left(G^{\prime}, \mathbf{s}\right)$.

- [GR4], where $G=\mathbf{p} \rightarrow \mathbf{q}:\left\{l_{i}: G_{i}\right\}_{i \in I}$, and $G^{\prime}=\mathbf{p} \rightarrow \mathbf{q}:\left\{l_{i}: G_{i}^{\prime}\right\}_{i \in I^{\prime}}$

By hypothesis, $\forall i \in I$. $\left(G_{i} \stackrel{l}{\longrightarrow} G_{i}^{\prime}\right)$ and $\mathbf{p} \neq \mathbf{q} \neq \operatorname{subj}(l)$.

If $G \uparrow \mathrm{r}$ exists, so does $G_{i} \uparrow \mathrm{r}$ for $i \in I$.

By assumption, $G \circledast \mathbf{s}$, so $\mathbf{s} \in\{\mathbf{p}, \mathbf{q}\} \wedge \wedge G_{i} \circledast \mathbf{s}$.

By induction, $\forall i \in I$. $\left(G_{i}^{\prime} \uparrow \mathrm{r}\right.$ exists $\left.\wedge G_{i}^{\prime} \circledast \mathrm{s}\right)$.

(1). To show $G^{\prime} \uparrow \mathrm{r}$, consider $\mathrm{r}$ by case:

- $\mathbf{r}=\mathbf{p}$ : Then $G^{\prime} \uparrow \mathbf{p}=\mathbf{q} \oplus\left\{l_{i}: G^{\prime} \uparrow \mathbf{p}\right\}_{i \in I}$.

- $\mathbf{r}=\mathbf{q}$ : Then $G^{\prime} \uparrow \mathbf{q}=\mathbf{p} \&\left\{l_{i}: G_{i}^{\prime} \uparrow \mathbf{q}\right\}_{i \in I}$.

- $\mathbf{r} \notin\{\mathbf{p}, \mathbf{q}\}$ : Then $G^{\prime} \uparrow \mathbf{r}=\prod_{i \in I} G_{i}^{\prime} \uparrow \mathbf{r}$. We know that $G \uparrow \mathbf{r}=\prod_{i \in I} G_{i} \uparrow \mathbf{r}$ exists. By Lemma C.1, $\prod_{i \in I} G_{i}^{\prime} \uparrow$ r exists too.
(2). We have $s \in\{\mathbf{p}, \mathbf{q}\}$ from assumption and $\bigwedge_{i \in I} G_{i}^{\prime} \circledast \mathbf{s}$ from induction, so $G^{\prime} \circledast$ s.

- [GR5], where $G=\mathbf{p} \rightsquigarrow \mathbf{q} . j:\left\{l_{i}: G_{i}\right\}_{i \in I}$,

and $G^{\prime}=\mathbf{p} \rightsquigarrow \mathbf{q} \cdot j:\left\{l_{i}: G_{i}^{\prime}\right\}_{i \in I^{\prime}}$.

By hypothesis, $G_{j} \stackrel{l}{\longrightarrow} G_{j}^{\prime}, \forall i \in I \backslash\{j\} . G_{i}^{\prime}=G_{i}$, and $\mathbf{q} \neq \operatorname{subj}(l)$.

If $G \uparrow \mathbf{r}$ exists, so does $G_{i} \uparrow \mathbf{r}$ for $i \in I$.

By assumption, $G \circledast \mathbf{s}$, so $\mathbf{s} \in\{\mathbf{p}, \mathbf{q}\} \wedge G_{j} \circledast \mathbf{s}$.

By induction on $G_{j} \stackrel{l}{\longrightarrow} G_{j}^{\prime}$ and hypothesis

$\forall i \in I \backslash\{j\} . G_{i}^{\prime}=G_{i}$, we get $\forall i \in I$. $\left(G_{i}^{\prime} \uparrow\right.$ r exists $\left.\wedge G_{i}^{\prime} \circledast \mathrm{s}\right)$.

(1). To show $G^{\prime} \uparrow \mathrm{r}$, consider $\mathrm{r}$ by case:

- $\mathbf{r}=\mathbf{p}$ : Then $G^{\prime} \uparrow \mathbf{p}=G_{j}^{\prime} \uparrow \mathbf{p}$.

- $\mathbf{r}=\mathbf{q}$ : Then $G^{\prime} \uparrow \mathbf{q}=\mathbf{p} \&\left\{l_{i}: G_{i}^{\prime} \uparrow \mathbf{q}\right\}_{i \in I^{\prime}}$.

- $\mathbf{r} \notin\{\mathbf{p}, \mathbf{q}\}$ : Then $G^{\prime} \uparrow \mathbf{r}=G_{j}^{\prime} \uparrow \mathbf{r}$.

(2). We have $s \in\{\mathbf{p}, \mathbf{q}\}$ from assumption and $G_{j}^{\prime} \circledast \mathrm{s}$ from induction, so $G^{\prime} \circledast \mathbf{s}$.

- [GR6], where $G=\mathbf{p}-\mathbf{t} \rightarrow \mathbf{q}:\left\{l_{i}: G_{i}\right\}_{i \in I}$,

$G^{\prime}=\mathbf{p} \underset{\mathbf{t}}{m} \mathbf{q} \cdot j:\left\{l_{i}: G_{i}\right\}_{i \in I}$, and $l=v i a\langle\mathbf{s}\rangle(\mathbf{p q} ! j)$.

By assumption, wellFormed ${ }^{R}(G, \mathbf{s})$, so $\mathbf{t}=\mathbf{s}$.

(1). We know $G \uparrow r$ by assumption. To show $G^{\prime} \uparrow r$, consider $r$ by case:

- $\mathbf{r}=\mathbf{p}$ : Then $G \uparrow \mathbf{p}=\mathbf{q} \oplus\langle\mathbf{s}\rangle\left\{l_{i}: G_{i} \uparrow \mathbf{p}\right\}_{i \in I}$, so $\forall i \in I . G_{i} \uparrow \mathbf{p}$ exists. $G^{\prime} \uparrow \mathbf{p}=G_{j} \uparrow \mathbf{p}$, which exists as $j \in I$.

- $\mathbf{r}=\mathbf{q}$ : Then $G^{\prime} \uparrow \mathbf{q}=\mathbf{p} \&\langle\mathbf{s}\rangle\left\{l_{i}: G_{i} \uparrow \mathbf{q}\right\}_{i \in I}=G \uparrow \mathbf{q}$, which exists.

- $\mathbf{r}=\mathbf{s}$ : Then $G \uparrow \mathbf{s}=\mathbf{p} \hookrightarrow \mathbf{q}:\left\{l_{i}: G_{i} \uparrow \mathbf{s}\right\}_{i \in I}$, so $\forall i \in I$. $G_{i} \uparrow \mathbf{s}$ exists.

$G^{\prime} \uparrow \mathbf{s}=\mathbf{p} \leftrightarrow \mathbf{q} . j:\left\{l_{i}: G_{i} \uparrow \mathbf{s}\right\}_{i \in I}$, which exists.

- $\mathbf{r} \notin\{\mathbf{p}, \mathbf{q}, \mathbf{s}\}$ : Then $G \uparrow \mathbf{r}=\prod_{i \in I} G_{i} \uparrow \mathbf{r}$, so $\forall i \in I$. $G_{i} \uparrow \mathbf{r}$ exists.

$G^{\prime} \uparrow \mathbf{r}=G_{j} \uparrow \mathbf{r}$, which exists as $j \in I$.

(2). We know $G \circledast \mathrm{s}$ by assumption. We deduce $G^{\prime} \circledast \mathrm{s}$ by consequence.

$$
\begin{aligned}
G \circledast \mathrm{s} & \Longrightarrow \mathbf{t}=\mathbf{s} \wedge \bigwedge_{i \in I} G_{i} \circledast \mathrm{s} \\
& \Longrightarrow \mathbf{t}=\mathbf{s} \wedge G_{j} \circledast \mathbf{s} \\
& \Longrightarrow G^{\prime} \circledast \mathbf{s}
\end{aligned}
$$

- [GR7], where $G=\mathbf{p} \underset{\mathbf{t}}{\rightsquigarrow \mathbf{q}} \cdot j:\left\{l_{i}: G_{i}\right\}_{i \in I}, G^{\prime}=G_{j}$ and $l=\mathrm{via}\langle\mathbf{s}\rangle(\mathbf{p q}$ ? $j)$.

(1). We know $G \uparrow r$ by assumption. To show $G^{\prime} \uparrow r$, consider $r$ by case:

By assumption, wellFormed ${ }^{R}(G, \mathbf{s})$, so $\mathbf{t}=\mathbf{s}$.

- $\mathbf{r}=\mathbf{p}$ : Then $G^{\prime} \uparrow \mathbf{p}=G_{j} \uparrow \mathbf{p}=G \uparrow \mathbf{p}$, which exists.

- $\mathbf{r}=\mathbf{q}$ : Then $G \uparrow \mathbf{q}=\mathbf{p} \&\langle\mathbf{s}\rangle\left\{l_{i}: G_{i} \uparrow \mathbf{q}\right\}_{i \in I}$, so $\forall i \in I . G_{i} \uparrow \mathbf{q}$ exists.

$G^{\prime} \uparrow \mathbf{q}=G_{j} \uparrow \mathbf{q}$, which exists as $j \in I$.

- $\mathbf{r}=\mathbf{s}$ : Then $G \uparrow \mathbf{s}=\mathbf{p} \leftrightarrow \mathbf{q} . j:\left\{l_{i}: G_{i}\lceil\mathbf{s}\}_{i \in I}\right.$, so $\forall i \in I$. $G_{i} \uparrow \mathbf{s}$ exists.

$G^{\prime}\left\lceil\mathbf{s}=G_{j}\lceil\mathbf{s}\right.$, which exists as $j \in I$.

- $\mathbf{r} \notin\{\mathbf{p}, \mathbf{q}, \mathbf{s}\}$ : Then $G \uparrow \mathbf{r}=\prod_{i \in I} G_{i} \uparrow \mathbf{r}$, so $\forall i \in I$. $G_{i} \uparrow \mathbf{r}$ exists.

$G^{\prime} \uparrow \mathbf{r}=G_{j} \uparrow \mathbf{r}$, which exists as $j \in I$.

(2). We know $G \circledast \mathrm{s}$ by assumption. We deduce $G^{\prime} \circledast \mathrm{s}$ by consequence.

$$
G \circledast \mathbf{s} \Longrightarrow \mathbf{s} \in\{\mathbf{p}, \mathbf{q}\} \wedge G_{j} \circledast \mathbf{s} \Longrightarrow G^{\prime} \circledast \mathbf{s}
$$

- [GR8], where $G=\mathbf{p}-\mathbf{t} \rightarrow \mathbf{q}:\left\{l_{i}: G_{i}\right\}_{i \in I}$, and $G^{\prime}=\mathbf{p}-\mathbf{t} \rightarrow \mathbf{q}:\left\{l_{i}: G_{i}^{\prime}\right\}_{i \in I^{\prime}}$ 
By hypothesis, $\forall i \in I .\left(G_{i} \stackrel{l}{\longrightarrow} G_{i}^{\prime}\right)$ and $\mathbf{p} \neq \mathbf{q} \neq \operatorname{subj}(l)$.

If $G \uparrow \mathrm{r}$ exists, so does $G_{i} \uparrow \mathrm{r}$ for $i \in I$.

By assumption, $G \circledast \mathrm{s}$, so $\mathbf{t}=\mathbf{s} \wedge \bigwedge_{i \in I} G_{i} \circledast \mathrm{s}$.

By induction, $\forall i \in I$. $\left(G_{i}^{\prime} \uparrow \mathrm{r}\right.$ exists $\left.\wedge G_{i}^{\prime} \circledast \mathrm{s}\right)$.

(1). To show $G^{\prime} \uparrow \mathrm{r}$, consider $\mathrm{r}$ by case:

- $\mathbf{r}=\mathbf{p}$ : Then $G^{\prime} \uparrow \mathbf{p}=\mathbf{q} \oplus\langle\mathbf{s}\rangle\left\{l_{i}: G^{\prime} \uparrow \mathbf{p}\right\}_{i \in I}$.

- $\mathbf{r}=\mathbf{q}$ : Then $G^{\prime} \uparrow \mathbf{q}=\mathbf{p} \&\langle\mathbf{s}\rangle\left\{l_{i}: G_{i}^{\prime} \uparrow \mathbf{q}\right\}_{i \in I}$.

- $\mathbf{r}=\mathbf{s}$ : Then $G^{\prime} \uparrow \mathbf{s}=\mathbf{p} \hookrightarrow \mathbf{q}:\left\{l_{i}: G_{i}^{\prime} \uparrow \mathbf{s}\right\}_{i \in I^{\prime}}$

- $\mathbf{r} \notin\{\mathbf{p}, \mathbf{q}, \mathbf{s}\}$ : Then $G^{\prime} \uparrow \mathbf{r}=\prod_{i \in I} G_{i}^{\prime} \uparrow \mathbf{r}$. We know that $G \uparrow \mathbf{r}=$ $\prod_{i \in I} G_{i} \uparrow$ r exists. By Lemma C. $1, \prod_{i \in I} G_{i}^{\prime} \uparrow$ r exists too.

(2). We have $\mathbf{t}=\mathbf{s}$ from assumption and $\bigwedge G_{i}^{\prime} \circledast \mathbf{s}$ from induction, so $G^{\prime} \circledast \mathbf{s}$.

- [GR9], where $G=\mathbf{p} \underset{\mathbf{t}}{\rightsquigarrow} \mathbf{q} \cdot j:\left\{l_{i}: G_{i}\right\}_{i \in I}$, and $G^{\prime}=\mathbf{p} \underset{\mathbf{t}}{\rightsquigarrow} \mathbf{q} \cdot j:\left\{l_{i}: G_{i}^{\prime}\right\}_{i \in I}$.

By hypothesis, $G_{j} \stackrel{l}{\longrightarrow} G_{j}^{\prime}$,

$\forall i \in I \backslash\{j\} . G_{i}^{\prime}=G_{i}$, and $\mathbf{q} \neq \operatorname{subj}(l)$.

If $G \uparrow \mathrm{r}$ exists, so does $G_{i} \uparrow \mathbf{r}$ for $i \in I$.

By assumption, $G \circledast \mathbf{s}$, so $\mathbf{t}=\mathbf{s} \wedge G_{j} \circledast \mathbf{s}$.

By induction on $G_{j} \stackrel{l}{\longrightarrow} G_{j}^{\prime}$ and hypothesis

$\forall i \in I \backslash\{j\} . G_{i}^{\prime}=G_{i}$, we get $\forall i \in I$. ( $G_{i}^{\prime} \uparrow \mathbf{r}$ exists $\left.\wedge G_{i}^{\prime} \circledast \mathbf{s}\right)$.

(1). To show $G^{\prime} \uparrow \mathrm{r}$, consider $\mathrm{r}$ by case:

- $\mathbf{r}=\mathbf{p}$ : Then $G^{\prime} \uparrow \mathbf{p}=G_{j}^{\prime} \uparrow \mathbf{p}$.

- $\mathbf{r}=\mathbf{q}$ : Then $G^{\prime} \uparrow \mathbf{q}=\mathbf{p} \&\langle\mathbf{s}\rangle\left\{l_{i}: G_{i}^{\prime} \uparrow \mathbf{q}\right\}_{i \in I}$.

- $\mathbf{r}=\mathbf{s}$ : Then $G^{\prime} \uparrow \mathbf{s}=\mathbf{p} \leftrightarrow \mathbf{q} . j:\left\{l_{i}: G_{i}^{\prime} \uparrow \mathbf{s}\right\}_{i \in I^{\prime}}$

- $\mathbf{r} \notin\{\mathbf{p}, \mathbf{q}, \mathbf{s}\}$ : Then $G^{\prime} \uparrow \mathbf{r}=G_{j}^{\prime} \uparrow \mathbf{r}$.

(2). We have $\mathrm{t}=\mathrm{s}$ from assumption and $G_{j}^{\prime} \circledast \mathrm{s}$ from induction, so $G^{\prime} \circledast$ s.

Lemma C.12 (Progress for Well-formed Global Types). Let $G$ be a global type. Suppose $G$ is well-formed with respect to some router $\mathbf{s}$, i.e. wellFormed $(G, s)$.

$$
(G=\text { end }) \vee \exists G^{\prime}, l .\left(G \stackrel{l}{\longrightarrow} G^{\prime}\right)
$$

Proof. The following is logically equivalent:

$$
(G \neq \text { end }) \Longrightarrow \exists G^{\prime}, l .\left(G \stackrel{l}{\longrightarrow} G^{\prime}\right) .
$$

We prove this by induction on the structure of $G$.

We do not consider $G=$ end by assumption.

We do not consider $G=\mathrm{t}$ as the type variable occurs free.

1. $G=\mu \mathrm{t} . G^{\prime \prime}$

t must occur in $G$, so $G[\mu \mathrm{t} . G / \mathrm{t}] \neq$ end.

By induction, $\exists G^{\prime}, l$. $\left(G[\mu \mathrm{t} . G / \mathrm{t}] \stackrel{l}{\longrightarrow} G^{\prime}\right)$.

Apply [GR3] to get $\exists G^{\prime}, l$. ( $\left.\mu \mathrm{t} . G \stackrel{l}{\longrightarrow} G^{\prime}\right)$.

2. $G=\mathbf{p} \rightarrow \mathbf{q}:\left\{l_{i}: G_{i}\right\}_{i \in I}$

Apply [GR1] to get $G \stackrel{\text { pq } ! j}{\longrightarrow}$ p $\rightsquigarrow \mathbf{q} \cdot j:\left\{l_{i}: G_{i}\right\}_{i \in I}$.

3. $G=\mathbf{p}-\mathbf{r} \rightarrow \mathbf{q}:\left\{l_{i}: G_{i}\right\}_{i \in I}$

By assumption, wellFormed ${ }^{R}(G, \mathbf{s})$, so $\mathbf{r}=\mathbf{s}$.

Apply [GR6] to get $G \stackrel{\text { via }\langle\mathbf{s}\rangle(\mathbf{p q} ! j)}{\longrightarrow}$ p $\underset{\mathbf{s}}{\rightsquigarrow \mathbf{q} .} j:\left\{l_{i}: G_{i}\right\}_{i \in I}$.
4. $G=\mathbf{p} \rightsquigarrow \mathbf{q} . j:\left\{l_{i}: G_{i}\right\}_{i \in I}$

Apply [GR2] to get $G \stackrel{\text { pq? } j}{\longrightarrow} G_{j}$.

5. $G=\mathbf{p} \underset{\mathbf{r}}{\leadsto}$ q. $j:\left\{l_{i}: G_{i}\right\}_{i \in I}$

By assumption, wellFormed ${ }^{R}(G, \mathbf{s})$, so $\mathbf{r}=\mathbf{s}$.

Apply [GR7] to get $G \stackrel{\mathrm{via}\langle\mathbf{s}\rangle(\mathbf{p q} ? j)}{\longrightarrow} G_{j}$.

\section{Proof of Theorem 4.11.}

Let $G$ be a global type, and s be a role. Then we have: wellFormed $(G) \Longleftrightarrow$ wellFormed $^{R}(\llbracket G, \mathrm{~s} \rrbracket, \mathrm{s})$

Proof. ( $\Longrightarrow$ ) Direct consequence of Lemmas C.3 and C.9 $(\Longleftarrow)$ By definition.

\section{C.4 Proof of Theorem 4.12}

Let $G, G^{\prime}$ be well-formed global types such that $G \stackrel{l}{\longrightarrow} G^{\prime}$ for some label $l$. Then we have:

$$
\forall l, \mathrm{~s} .\left(G \stackrel{l}{\longrightarrow} G^{\prime} \Longleftrightarrow \llbracket G, \mathrm{~s} \rrbracket \stackrel{\llbracket l, \mathrm{~s} \rrbracket}{\longrightarrow} \llbracket G^{\prime}, \mathrm{s} \rrbracket\right)
$$

Proof. By rule induction on $G \stackrel{l}{\longrightarrow} G^{\prime}$. Take arbitrary router role s.

- [GR1], where $G=\mathbf{p} \rightarrow \mathbf{q}:\left\{l_{i}: G_{i}\right\}_{i \in I}$,

$G^{\prime}=\mathbf{p} \rightsquigarrow \mathbf{q} \cdot j:\left\{l_{i}: G_{i}\right\}_{i \in I}$,

and $l=\mathrm{pq} ! j$.

To show $\llbracket G, \mathrm{~s} \rrbracket \stackrel{\llbracket l, \mathrm{~s} \rrbracket}{\longrightarrow} \llbracket G^{\prime}, \mathrm{s} \rrbracket$, consider s by case: $-s \in\{p, q\}$ : Then we have

$$
\begin{aligned}
\llbracket G, \mathbf{s} \rrbracket & =\mathbf{p} \rightarrow \mathbf{q}:\left\{l_{i}: \llbracket G_{i}, \mathbf{s} \rrbracket\right\}_{i \in I} \\
\llbracket G^{\prime}, \mathbf{s} \rrbracket & =\mathbf{p} \leadsto \mathbf{q} \cdot j:\left\{l_{i}: \llbracket G_{i}, \mathbf{s} \rrbracket\right\}_{i \in I} \\
\llbracket l, \mathbf{s} \rrbracket & =\mathbf{p q} ! j
\end{aligned}
$$

The encoded transition is possible using [GR1]. - $s \notin\{p, q\}$ : Then we have

$$
\begin{aligned}
\llbracket G, \mathbf{s} \rrbracket & =\mathbf{p}-\mathbf{s} \rightarrow \mathbf{q}:\left\{l_{i}: \llbracket G_{i}, \mathbf{s} \rrbracket\right\}_{i \in I} \\
\llbracket G^{\prime}, \mathbf{s} \rrbracket & =\underset{\mathbf{s}}{\mathbf{p}} \mathbf{q} \cdot j:\left\{l_{i}: \llbracket G_{i}, \mathbf{s} \rrbracket\right\}_{i \in I} \\
\llbracket l, \mathbf{s} \rrbracket & =\operatorname{via}\langle\mathbf{s}\rangle(\mathbf{p q} ! j)
\end{aligned}
$$

The encoded transition is possible using [GR6].

- [GR2], where $G=\mathbf{p} \rightsquigarrow$ q. $j:\left\{l_{i}: G_{i}\right\}_{i \in I}, G^{\prime}=G_{j}, l=\mathbf{p q}$ ? $j$.

We know $\llbracket G^{\prime}, \mathrm{s} \rrbracket=\llbracket G_{j}, \mathrm{~s} \rrbracket$

To show $\llbracket G, s \rrbracket \stackrel{\llbracket l, s \rrbracket}{\longrightarrow} \llbracket G^{\prime}, s \rrbracket$, consider s by case: $-s \in\{p, q\}$ : Then we have

$$
\begin{aligned}
\llbracket G, \mathbf{s} \rrbracket & =\mathbf{p} \rightsquigarrow \mathbf{q} \cdot j:\left\{l_{i}: \llbracket G_{i}, \mathbf{s} \rrbracket\right\}_{i \in I} \\
\llbracket l, \mathbf{s} \rrbracket & =\mathbf{p q} ? j
\end{aligned}
$$

The encoded transition is possible using [GR2]. - $\mathbf{s}\{\{\mathbf{p}, \mathbf{q}\}$ : Then we have

$$
\begin{aligned}
\llbracket G, \mathbf{s} \rrbracket & =\mathbf{p} \underset{\mathrm{s}}{m} \mathbf{q} \cdot j:\left\{l_{i}: \llbracket G_{i}, \mathbf{s} \rrbracket\right\}_{i \in I} \\
\llbracket l, \mathbf{s} \rrbracket & =\operatorname{via}\langle\mathbf{s}\rangle(\mathbf{p q} ? j)
\end{aligned}
$$

The encoded transition is possible using [GR7].

- [Gr3], where $G=\mu$ t. $G^{\prime \prime}$.

By hypothesis, $G^{\prime \prime}\left[\mu \mathrm{t} . G^{\prime \prime} / \mathrm{t}\right] \stackrel{l}{\longrightarrow} G^{\prime}$.

By induction, $\llbracket G^{\prime \prime}\left[\mu \mathrm{t} . G^{\prime \prime} / \mathbf{t}\right], \mathrm{s} \rrbracket \stackrel{\llbracket l, \mathrm{~s} \rrbracket}{\longrightarrow} \llbracket G^{\prime}, \mathbf{s} \rrbracket$.

By Lemma C.4, $\llbracket G^{\prime \prime}\left[\mu \mathrm{t} . G^{\prime \prime} / \mathrm{t}\right], \mathrm{s} \rrbracket=\llbracket G^{\prime \prime}, \mathrm{s} \rrbracket\left[\mu \mathrm{t} . \llbracket G^{\prime \prime}, \mathrm{s} \rrbracket / \mathrm{t}\right]$. We know $\llbracket G, \mathrm{~s} \rrbracket=\llbracket \mu \mathrm{t} \cdot G^{\prime \prime}, \mathrm{s} \rrbracket=\mu \mathrm{t} \cdot \llbracket G^{\prime \prime}, \mathrm{s} \rrbracket$. 
The encoded transition is possible using [GR3] as shown:

$$
\frac{\llbracket G^{\prime \prime}, \mathrm{s} \rrbracket\left[\mu \mathrm{t} \cdot \llbracket G^{\prime \prime}, \mathrm{s} \rrbracket / \mathrm{t}\right] \stackrel{\llbracket l, \mathrm{~s} \rrbracket}{\longrightarrow} \llbracket \llbracket G^{\prime}, \mathrm{s} \rrbracket}{\mu \mathrm{t} \cdot \llbracket G^{\prime \prime}, \mathrm{s} \rrbracket \stackrel{\llbracket l, \mathrm{~s} \rrbracket}{\longrightarrow} \llbracket G^{\prime}, \mathrm{s} \rrbracket} \text { [GR3] }
$$

- [GR4], where $G=\mathbf{p} \rightarrow \mathbf{q}:\left\{l_{i}: G_{i}\right\}_{i \in I}, G^{\prime}=\mathbf{p} \rightarrow \mathbf{q}:\left\{l_{i}: G_{i}^{\prime}\right\}_{i \in I}$.

By hypothesis, $\forall i \in I$. $G_{i} \stackrel{l}{\longrightarrow} G_{i}^{\prime}$ and $\operatorname{subj}(l) \notin\{\mathbf{p}, \mathbf{q}\}$.

By induction, $\forall i \in I .\left(\llbracket G_{i}, \mathrm{~s} \rrbracket \stackrel{\llbracket l, \mathrm{~s} \rrbracket}{\longrightarrow} \llbracket G_{i}^{\prime}, \mathrm{s} \rrbracket\right)$.

By definition of $\operatorname{subj}(\cdot), \operatorname{subj}(\llbracket l, s \rrbracket) \notin\{\mathbf{p}, \mathbf{q}\}$.

To show $\llbracket G, s \rrbracket \stackrel{\llbracket l, s \rrbracket}{\longrightarrow} \llbracket G^{\prime}, s \rrbracket$, consider s by case:

$-s \in\{p, q\}$ : Then we have

$$
\begin{aligned}
\llbracket G, \mathbf{s} \rrbracket & =\mathbf{p} \rightarrow \mathbf{q}:\left\{l_{i}: \llbracket G_{i}, \mathbf{s} \rrbracket\right\}_{i \in I} \\
\llbracket G^{\prime}, \mathbf{s} \rrbracket & =\mathbf{p} \rightarrow \mathbf{q}:\left\{l_{i}: \llbracket G_{i}^{\prime}, \mathbf{s} \rrbracket\right\}_{i \in I}
\end{aligned}
$$

The encoded transition is possible using [GR4].

- $\mathbf{s} \notin\{\mathbf{p}, \mathbf{q}\}$ : Then we have

$$
\begin{aligned}
\llbracket G, \mathbf{s} \rrbracket & =\mathbf{p}-\mathbf{s} \rightarrow \mathbf{q}:\left\{l_{i}: \llbracket G_{i}, \mathbf{s} \rrbracket\right\}_{i \in I} \\
\llbracket G^{\prime}, \mathbf{s} \rrbracket & =\mathbf{p}-\mathbf{s} \rightarrow \mathbf{q}:\left\{l_{i}: \llbracket G_{i}^{\prime}, \mathbf{s} \rrbracket\right\}_{i \in I}
\end{aligned}
$$

The encoded transition is possible using [GR8].

- [GR5], where $G=\mathbf{p} \rightsquigarrow \mathbf{q} \cdot j:\left\{l_{i}: G_{i}\right\}_{i \in I}$,

$G^{\prime}=\mathbf{p} \rightsquigarrow \mathbf{q} \cdot j:\left\{l_{i}: G_{i}^{\prime}\right\}_{i \in I}$.

By hypothesis, $G_{j} \stackrel{l}{\longrightarrow} G_{j}^{\prime}$, p' $\neq \operatorname{subj}(l)$, and $\forall i \in I \backslash\{j\}$. $G_{i}^{\prime}=G_{i}$.

By induction, $\llbracket G_{j}, \mathrm{~s} \rrbracket \stackrel{\llbracket l, \mathrm{~s} \rrbracket}{\longrightarrow} \llbracket G_{j}^{\prime}, \mathrm{s} \rrbracket$.

By definition of a set of subjects, we have: $\operatorname{subj}(\llbracket l, s \rrbracket) \neq \mathbf{q}$.

To show $\llbracket G, \mathrm{~s} \rrbracket \stackrel{\llbracket l, \mathrm{~s} \rrbracket}{\longrightarrow} \llbracket G^{\prime}, \mathrm{s} \rrbracket$, consider s by case:

$-s \in\{p, q\}$ : Then we have

$$
\begin{aligned}
\llbracket G, \mathbf{s} \rrbracket & =\mathbf{p} \leadsto \mathbf{q} \cdot j:\left\{l_{i}: \llbracket G_{i}, \mathbf{s} \rrbracket\right\}_{i \in I} \\
\llbracket G^{\prime}, \mathbf{s} \rrbracket & =\mathbf{p} \rightsquigarrow \mathbf{q} \cdot j:\left\{l_{i}: \llbracket G_{i}^{\prime}, \mathbf{s} \rrbracket\right\}_{i \in I}
\end{aligned}
$$

The encoded transition is possible using [GR5].

- $s \notin\{\mathbf{p}, \mathbf{q}\}$ : Then we have

$$
\begin{gathered}
\llbracket G, \mathbf{s} \rrbracket=\mathbf{p} \underset{\mathbf{s}}{\rightsquigarrow \mathbf{q}} \cdot j:\left\{l_{i}: \llbracket G_{i}, \mathbf{s} \rrbracket\right\}_{i \in I} \\
\llbracket G^{\prime}, \mathbf{s} \rrbracket=\mathbf{p} \underset{\mathbf{s}}{\rightsquigarrow \mathbf{q}} \cdot j:\left\{l_{i}: \llbracket G_{i}^{\prime}, \mathbf{s} \rrbracket\right\}_{i \in I}
\end{gathered}
$$

The encoded transition is possible using [GR9].

$(\Longleftarrow)$ direction is similar by rule induction on

$$
\llbracket G, \mathrm{~s} \rrbracket \stackrel{\llbracket l, \mathrm{~s} \rrbracket}{\longrightarrow} \llbracket G^{\prime}, \mathrm{s} \rrbracket .
$$

\section{Appendix for $§ 5$}

We include two more studies: Battleships and Travel Agency.

Battleships. We implement the Battleships board gamebetween two players, as used by King et al. [20]. The initialisation phase involves both players placing rectangular battleships on a $2 \mathrm{D}$ grid. The session proceeds to the game loop, where players take turns to guess the location of their opponent's ships, where the server responds with a hit or a miss. The game loop continues until all ships of one player have been sunk.

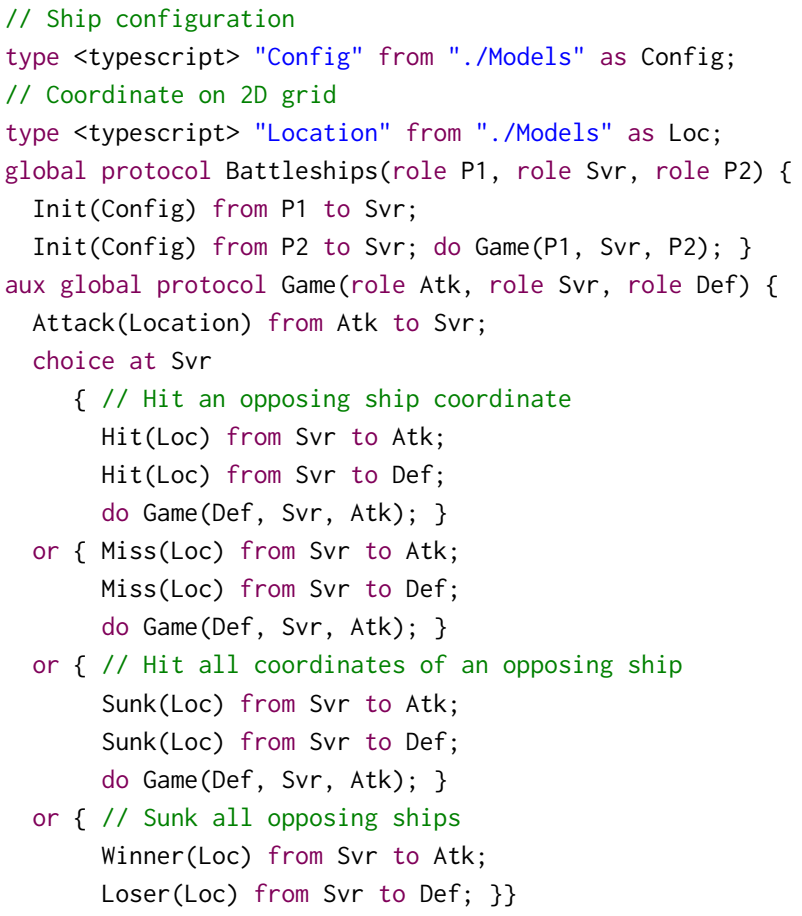

By interpreting the session ID (generated for Svr by STScript) as an unique game identifier, the developer can keep track of concurrent game sessions very easily. As the generated session runtime for Node.js needs to be initialised by a function that is parameterised by the game ID and returns the initial state, the game ID is bound inside the closure. This means that the invocation of callbacks (as part of the game logic) can access the game ID of the current game, so the developer can update the application state of the corresponding game.

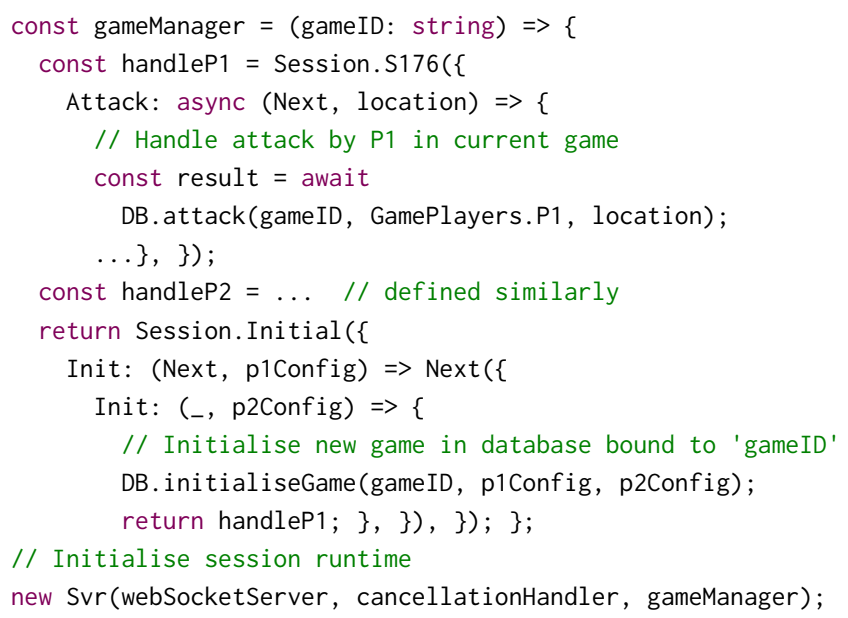

Because the API for the session cancellation handler also exposes the session ID of the cancelled session, the Svr can use the session ID to free up resources allocated to the corresponding game session accordingly. In fact, given that the API also exposes the role that initiated the cancellation, the Svr could identify which player forfeited the game and update leaderboard details to reflect the forfeit.

const cancellationHandler $=$ async ( 
cancel: Cancellation,

register: ReceiveStateHandler): void; \};

interface ITerminal \{ readonly type: 'Terminal'; \};

export type Type $=$ ISend | IReceive | ITerminal;

export class S40 implements ISend \{

readonly type: 'Send' = 'Send';

constructor(public handler: Handler.S40) \{\}

performSend(next: StateTransitionHandler, cancel: Cancellation, send: SendStateHandler) \{ const thunk $=($

[label, payload, succ]: FromPromise $<$ Handler.S40>) $\Rightarrow$ \{ send(Role.Peers.A, label, payload);

return next (succ); \};

if (this.handler instanceof Promise) \{ this.handler. then (thunk). catch (cancel); \}

else $\{\operatorname{try}\{$ thunk(this.handler); \}

catch (error) $\{$ cancel(error); $\}\}\}\}$;

export class S38 implements IReceive \{

readonly type: 'Receive' = 'Receive';

constructor(public handler: Handler.S38) \{\}

prepareReceive(next: StateTransitionHandler, cancel: Cancellation, register: ReceiveStateHandler) \{

const onReceive $=$ (message: any) $\Rightarrow\{$

const parsed $=$ JSON. parse(message) as Message.S38;

switch (parsed.label) \{

case "Query": $\{\operatorname{try}\{$

const successor $=$ this.handler[parsed.label $]($

Factory.S40, ...pparsed.payload);

if (successor instanceof Promise) \{ successor.then (next). $\operatorname{catch}($ cancel);

else $\{$ next(successor); \}

\} catch (error) \{ cancel(error); \}

return; \}\}\};

register(Role.Peers.A, onReceive); \}\};

The handler for each EFSM state is used to instantiate its State class instance, which is used by the session runtime to trigger the communication action. For send states, the runtime triggers the performSend method (Line 20) to perform the send using the label and payload defined in the handler (Line 24). For receive states, the State class instance registers the handler to the runtime, so the handler can be invoked when the message is received. The State class will check whether the handler is defined asynchronously (i.e. a TypeScript Promise) and invoke the handler accordingly.

\section{namespace Factory}

type S40_Available =

| [Message.S40_Available['payload'], (Next: typeof S41) $\Rightarrow$ State.S41]

I [Message.S40_Available['payload'], State.S41];

function S40_Available(

payload: Message.S40_Available['payload'],

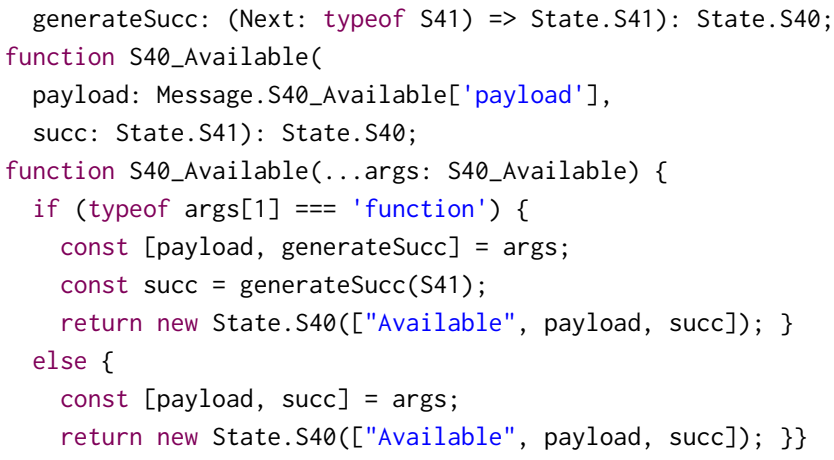

The Factory namespace exposes developer-friendly APIs for instantiating the State class instance. The factory API for a send state is an object literal defining labelled callbacks for each possible selection. Each callback is parameterised by the message payload and successor state. The factory API for a receive state is an alias for the constructor function of the corresponding State class. Initial and Terminal aliases are also exported as convenient references to the initial and terminal EFSM state respectively.

\section{E.2 Server Role S: API Usage}

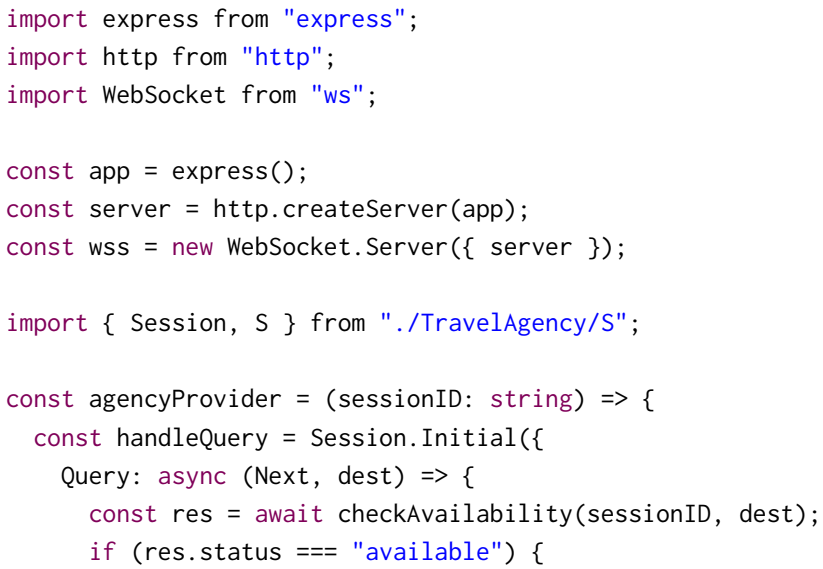




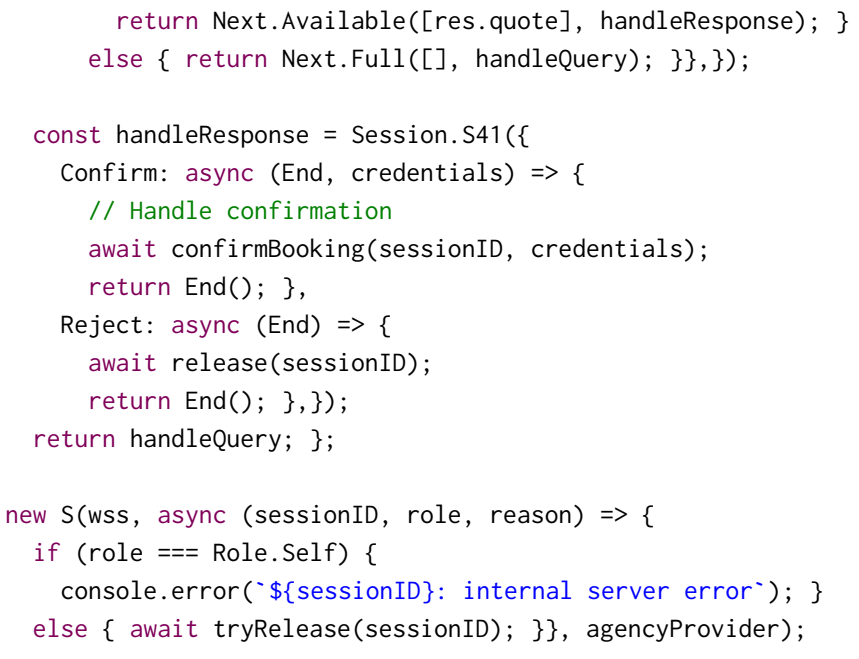

The developer instantiates the session (Line 29) using the WebSocket server, cancellation handler, and the EFSM implementation - a function to be invoked for every new session, parameterised by the session ID, and returns the State class instance of the initial state. Line 12 implements the API generated for a receive state, specifying how to handle a Query message. Line 16 implements the API generated for a send state - send the Available message with the price and proceed to the handleResponse continuation.

\section{E.3 Client Role B: Generated APIs}

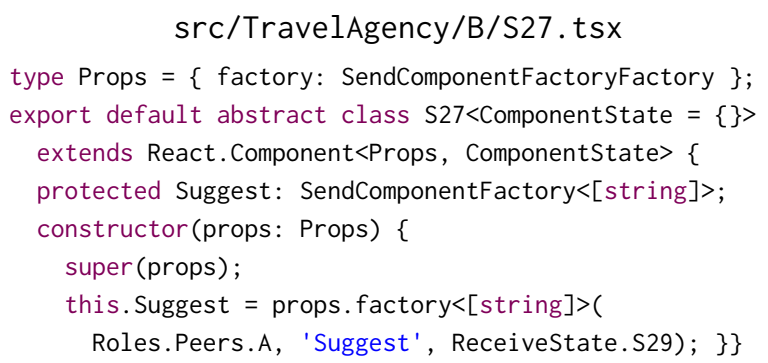

The generated React component for a send state receives a factory function from the session runtime to generate component factories for each permitted selection. Line 7 reads, "generate a component factory which sends a message (labelled Suggest to role A with one string-typed payload) and transitions to state S29". The component factory is defined as a protected property to allow access by subclasses implemented by the developer.

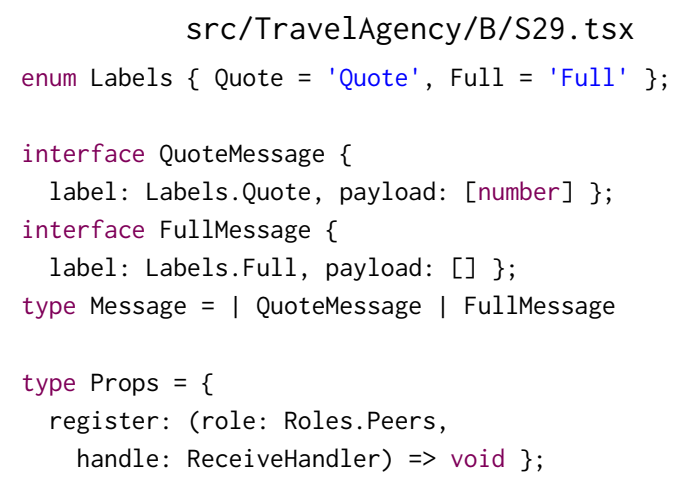

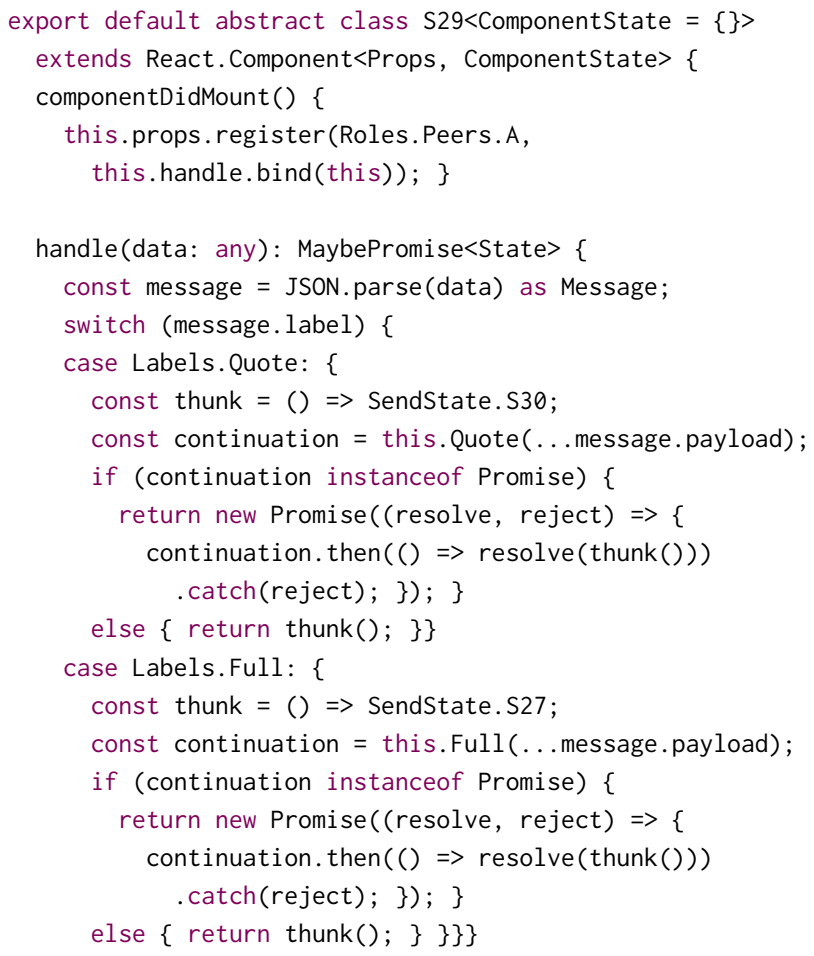

abstract Quote(payload1: number, ): MaybePromise<void>; abstract Full(): MaybePromise<void $>$; \}

The generated React component for a receive state registers a message handler (Line 19) to the session runtime component, to be invoked on a WebSocket onmessage event. An abstract method is exposed for each possible branch (Lines 39 and 40), requiring the developer to explicitly handle the received message. When invoked, the message handler parses the WebSocket message and invokes the abstract method (implemented by the developer) corresponding to the label of the received message. The message handler returns the successor state to the runtime to advance the EFSM.

\section{E.4 Client Role B: API Usage}

\section{src/components/MakeSuggestion.tsx}

import \{ S27 \} from "../../TravelAgency/B"; export default class MakeSuggestion extends S27 \{ static contextType = FriendState; declare context: React. ContextType<typeof FriendState>;

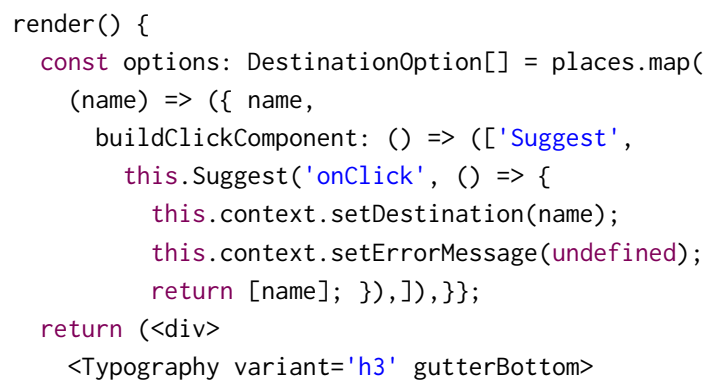




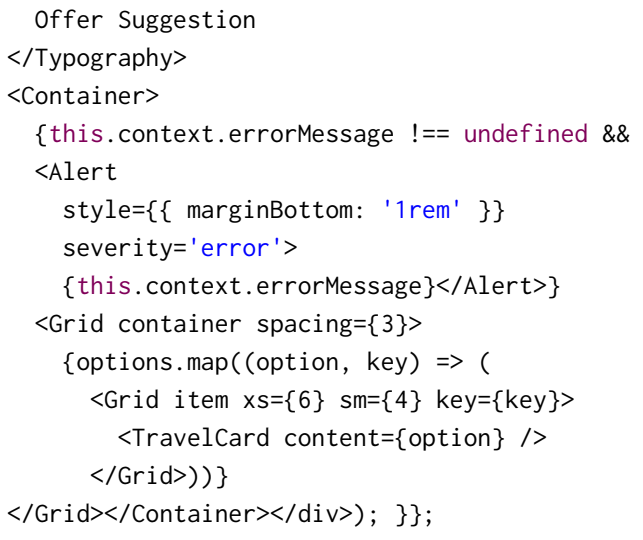

The developer implements the send state $\$ 27$ by extending from the corresponding abstract class, and uses the component factory property to generate UI components that are bound to the communication action. Line 10 creates a React component that sends the Suggest message on a click event, for each destination option in places.

\section{Wai tResponse. tsx}

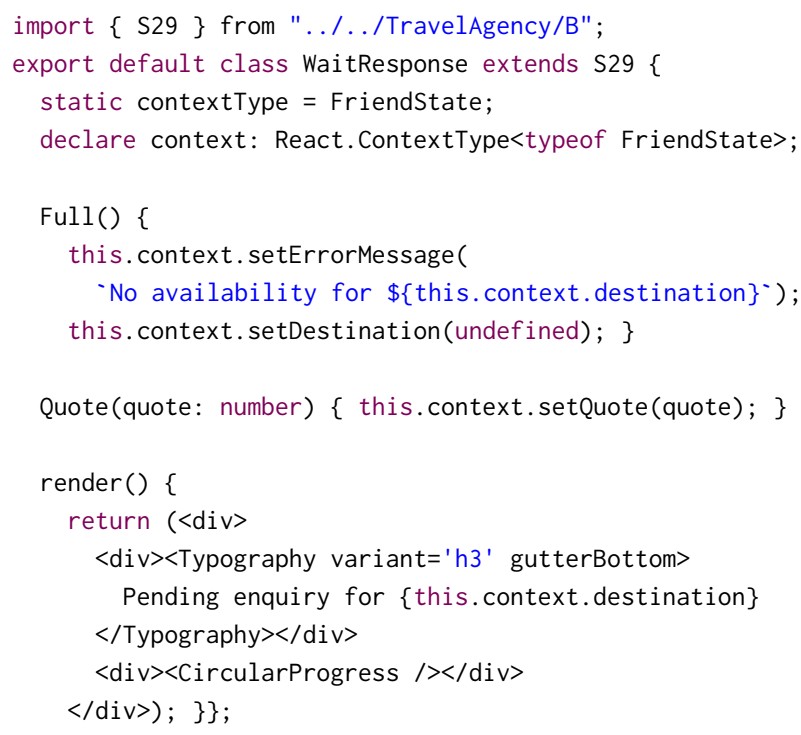

The developer implements the receive state S29 by extending from the corresponding abstract class, and implements the required abstract methods to define how to handle a Full message (Line 6) or a Quote message (Line 11).

\section{FriendView. tsx}

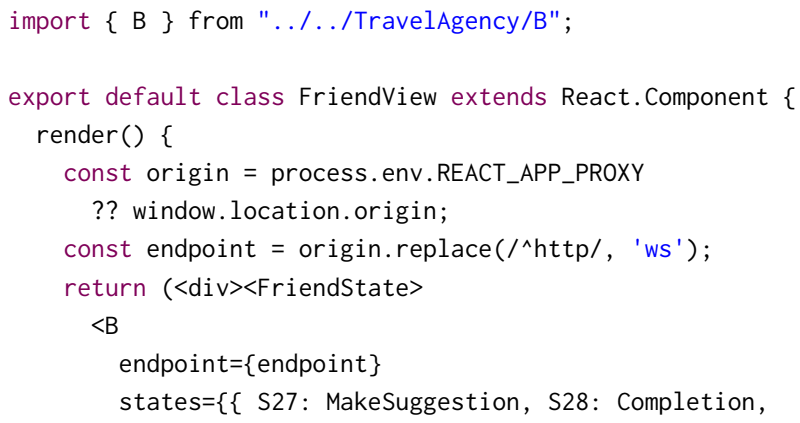

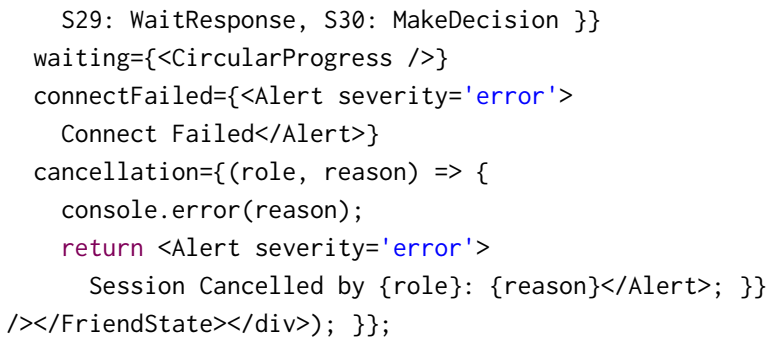

The developer instantiates the session (Line 9) by supplying: the WebSocket endpoint (Line 10), a React component to render whilst waiting (Line 13), a React component to render on a connection failure (Line 14), a function to build a React component to respond to session cancellation (Line 16), and an object literal (Line 11) mapping each EFSM state to the corresponding subclass implemented by the developer. 